\title{
Group-by-Group Probabilistic Bisimilarities and Their Logical Characterizations *
}

\author{
Marco Bernardo ${ }^{1}$ Rocco De Nicola ${ }^{2}$ Michele Loreti $^{3}$ \\ ${ }^{1}$ Dipartimento di Scienze di Base e Fondamenti - Università di Urbino - Italy \\ ${ }^{2}$ IMT - Institute for Advanced Studies Lucca - Italy \\ ${ }^{3}$ Dipartimento di Statistica, Informatica, Applicazioni - Università di Firenze - Italy
}

\begin{abstract}
We provide two interpretations, over nondeterministic and probabilistic processes, of PML, the probabilistic version of HennessyMilner logic used by Larsen and Skou to characterize bisimilarity of probabilistic processes without internal nondeterminism. We also exhibit two new bisimulation-based equivalences, which are in full agreement with the two different interpretations of PML. The new equivalences are coarser than the bisimilarity for nondeterministic and probabilistic processes proposed by Segala and Lynch, which instead is in agreement with a version of Hennessy-Milner logic extended with an additional probabilistic operator interpreted over state distributions rather than over individual states. The modal logic characterizations provided for the new equivalences thus offer a uniform framework for reasoning on purely nondeterministic processes, reactive probabilistic processes, and nondeterministic and probabilistic processes.
\end{abstract}

\section{Introduction}

Modal logics and behavioral equivalences play a key rôle in the specification and verification of concurrent systems. The former are useful for model checking, in that they can be employed for specifying the properties to be verified. The latter are ancillary to the former, in the sense that they enable the transformation/minimization of the models to be checked while guaranteeing that specific properties are preserved.

Because of this, whenever a new behavioral relation is proposed, the quest starts for the associated modal logic, i.e., for a logic such that two systems are behaviorally equivalent if and only if they satisfy the same logical formulae. The first result along this line is due to Hennessy and Milner [12]. They showed that bisimilarity over fully nondeterministic processes, modeled as a labeled transition system (LTS) [15], is in full agreement with a very simple modal logic, now known as HML. This logic has only four operators: true, $\cdot \wedge \cdot, \neg \cdot$, and $\langle a\rangle \cdot$, the last one called diamond and used to describe the existence of $a$-labeled transitions. After this result, whenever any of the many quantitative variants of process description

* Work partially supported by the FP7-IST-FET Project ASCENS, grant no. 257414, and the MIUR-PRIN Project CINA. 
languages and process models has been introduced, other behavioral equivalences and modal logics have been defined and analogous results have been established to handle features such as probability and time.

Most of the works along the lines outlined above take as starting point a behavioral equivalence and then look for the logic in agreement with it. Obviously, it is also interesting, once one has fixed a model and a logic to reason about it, to find out the "right" behavioral relation. A first work in this direction was [4]; it showed that bisimilarity and stuttering bisimilarity are, respectively, in full agreement with the logical equivalences induced by $\mathrm{CTL}^{*}$ and by $\mathrm{CTL}^{*}$ without the next-time operator when interpreted over Kripke structures (state-labeled transition systems) [5]. In a subsequent work, it was shown that the equivalence induced by the probabilistic temporal logic PCTL*$^{*}$, interpreted over probabilistic Kripke structures, coincides with probabilistic bisimilarity [1]. A more recent work is [25], which introduces new probabilistic bisimilarities that are in full agreement with the logical equivalences induced by PCTL, PCTL*, and their variants without the next-time operator interpreted over nondeterministic and probabilistic Kripke structures [3].

In this paper, we concentrate on the results obtained for extended LTS models that have been developed to deal with probabilistic systems. We look for bisimilarities that are in agreement with a probabilistic variant of HML known as PML $[16,17]$. This logic is obtained by simply decorating the diamond operator with a probability bound. Formula $\langle a\rangle_{p} \phi$ is satisfied by state $s$ if an $a$-labeled transition is possible from $s$ after which a set of states satisfying $\phi$ is reached with probability at least $p$.

Modal logic characterizations for probabilistic bisimilarities have been studied for the first time by Larsen and Skou $[16,17]$. They introduced a probabilistic bisimilarity for reactive probabilistic processes [27] and showed that the considered probabilistic bisimilarity is in full agreement with PML. Subsequently, Desharnais et al. [9] showed that PML without negation is sufficient to characterize probabilistic bisimilarity for the same class of processes. Reactive probabilistic processes being LTS-based models where (i) every action-labeled transition reaches a probability distribution over states and (ii) the actions labeling transitions departing from the same state are all different from each other.

Segala and Lynch [22] defined, instead, a probabilistic bisimilarity for a more expressive model that also admits internal nondeterminism, i.e., the possibility for a state to have several outgoing transitions labeled with the same action. For this probabilistic bisimilarity over nondeterministic and probabilistic processes, Segala and collaborators $[18,13]$ exhibited a logical characterization in terms of an extension of HML, in which formulae satisfaction is defined over probability distributions on states rather than over single states. The logic is obtained from HML by giving the diamond operator a universal interpretation (all states in the support of a distribution must satisfy the formula) and by adding a unary operator $[\cdot]_{p}$ such that $[\phi]_{p}$ is true on a state distribution whenever the probability of the set of states that satisfy formula $\phi$ is at least $p$. Recently, Crafa and Ranzato [6] showed an equivalent formulation of the logic that retrieves the HML 

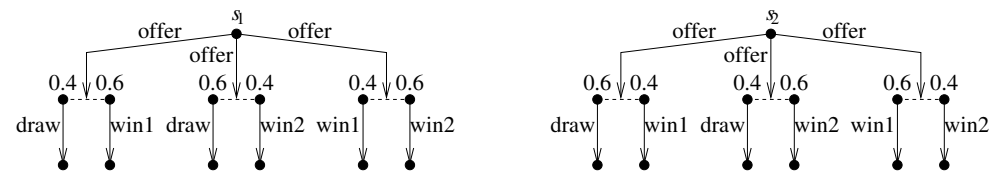

Fig. 1. Two games guaranteeing the same winning probabilities $\left(\sim_{\mathrm{PB}, \mathrm{gbg},=}\right)$.

interpretation of the diamond operator by lifting the transition relation to state distributions. Following a similar lifting, Hennessy [11] proposed an alternative logical characterization based on what he calls pHML, where a binary operator - $\oplus_{p}$. is added to HML (instead of the unary operator $[\cdot]_{p}$ ) such that $\phi_{1} \oplus_{p} \phi_{2}$ asserts decomposability of a state distribution to satisfy the two subformulae.

Now, the difference between PML and the two probabilistic extensions of HML in [18] and [11] is quite striking. It is thus interesting to understand whether such a difference is due to the different expressive power of the models in [16] and [22] - i.e., the absence or the presence of internal nondeterminism - or to the way probabilistic bisimilarity was defined on those two models. Since in [18] it was shown that PML characterizes the probabilistic bisimilarity over processes alternating nondeterminism and probability defined in [10,19], we feel it is worth exploring alternative definitions of probabilistic bisimilarity rather than alternative models.

The aim of this paper is to show that it is possible to define new probabilistic bisimilarities for non-alternating nondeterministic and probabilistic processes [21] that are characterized by PML. Our result is somehow similar to the one established in [25], where new probabilistic bisimilarities over nondeterministic and probabilistic Kripke structures were exhibited that are characterized by PCTL and its variants. In both cases, the starting point for defining the new probabilistic bisimilarities is the consideration (see also [7]) that sometimes the definition of Segala and Lynch [22] might be over discriminating and thus differentiate processes that, according to intuition, should be identified.

Indeed, to compare systems where both nondeterminism and probabilistic choices coexist, in $[21,22]$ the notion of scheduler (or adversary) is used to resolve internal nondeterminism. A scheduler can be viewed as an external entity that selects the next action to perform according to the current state and the past history. When a scheduler is applied to a system, a fully probabilistic model called a resolution is obtained. The basic idea is deeming equivalent two systems if and only if for each resolution of one system (the challenger) there exists a resolution of the other (the defender) such that the two resolutions are probabilistic bisimilar in the sense of [16] (fully matching resolutions).

Let us consider two scenarios modeling the offer to Player1 and Player2 of three differently biased dice. The game is conceived in such a way that if the outcome of a throw gives 1 or 2 then Player 1 wins, while if the outcome is 5 or 6 Player2 wins. In case of 3 or 4 , the result is a draw. The two scenarios are reported in Fig. 1. For instance, with the biased die associated with the leftmost branch of the first scenario, it happens that 3 or 4 (draw) will appear 

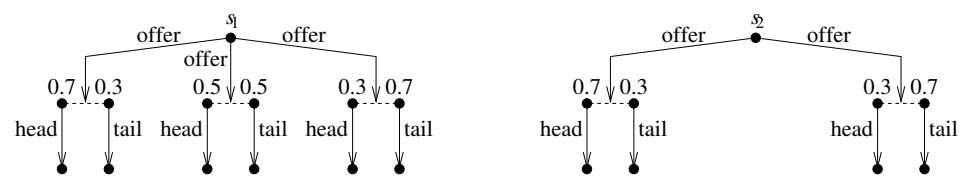

Fig. 2. Two games guaranteeing the same extremal head/tail probabilities $\left(\sim_{\mathrm{PB}, \mathrm{gbg}, \leq}\right)$.

with probability 0.4 , while 1 or 2 (Player 1 wins) will appear with probability 0.6 . Numbers 5 and 6 will never appear (no chance for Player2 to win).

The probabilistic bisimilarity proposed in [22] differentiates the models in Fig. 1 even if in both scenarios each player has the same set of probabilities of winning/drawing/losing, which is $\{0.6,0.4,0\}$. To identify these systems, from a bisimulation perspective it is needed to weaken the impact of schedulers. Indeed, while in [22] the challenger and the defender must stepwise behave the same along two fully matching resolutions, here, in the same vein as [26], we admit bisimulation games with partially matching resolutions.

Other two systems differentiated (under deterministic schedulers) by the probabilistic bisimilarity in [22] are those in Fig. 2. In the first scenario, the two players are offered a choice among a fair coin and two biased ones. In the second scenario, the players can simply choose between the two biased coins of the former scenario. In both scenarios, Player1 wins with head while Player2 wins with tail. In our view, the two scenarios could be identified if what matters is that in both of them each player has exactly the same extremal-i.e., minimal and maximal - probabilities of winning (0.3 and 0.7).

The first probabilistic bisimilarity we will introduce - denoted by $\sim_{\mathrm{PB}, \mathrm{gbg},=}=$ identifies the two systems in Fig. 1, but distinguishes those in Fig. 2. Our second probabilistic bisimilarity - denoted by $\sim_{\mathrm{PB}, \mathrm{gbg}, \leq}-$ instead identifies both the two systems in Fig. 1 and the two systems in Fig. 2. Notably, the same identifications are induced by one of the probabilistic bisimilarities in [25]. Indeed, once the appropriate transformations (eliminating actions from transitions and labeling each state with the set of possible next-actions) are applied to get nondeterministic and probabilistic Kripke structures from the four systems in Figs. 1 and 2, we have that no PCTL* formula distinguishes the two systems in Fig. 1 and the two systems in Fig. 2. However, it is worth pointing out that neither $\sim_{\mathrm{PB}, \mathrm{gbg},}=$ nor $\sim_{\mathrm{PB}, \mathrm{gbg}, \leq}$ coincides with the probabilistic bisimilarities in [25].

We shall show that $\sim_{\mathrm{PB}, \mathrm{gbg}, \leq} \leq$ is precisely characterized by the original PML as defined by Larsen and Skou [16,17], with the original interpretation of the diamond operator: state $s$ satisfies $\langle a\rangle_{p} \phi$ if $s$ has an $a$-transition that reaches with probability at least $p$ a set of states satisfying $\phi$. In contrast, $\sim_{\mathrm{PB}, \mathrm{gbg},=}$ is characterized by a variant of PML having an interval-based operator $\langle a\rangle_{\left[p_{1}, p_{2}\right]}$. instead of $\langle a\rangle_{p}:$ : state $s$ satisfies $\langle a\rangle_{\left[p_{1}, p_{2}\right]} \phi$ if $s$ has an $a$-transition that reaches with probability between $p_{1}$ and $p_{2}$ a set of states satisfying $\phi$. We shall refer to the interpretation of these two diamond operators as existential because it simply requires that there exists a way to resolve internal nondeterminism that guarantees satisfaction of formula $\phi$ within a certain probability range. 
For both logics, we shall also provide an alternative interpretation of the diamond operator, which is inspired by the actual interpretation of PCTL* in [3]. We shall call universal this interpretation that might appear more appropriate in a nondeterministic and probabilistic setting. With this interpretation, state

$s$ satisfies $\langle a\rangle_{p} \phi$ (resp. $\left.\langle a\rangle_{\left[p_{1}, p_{2}\right]} \phi\right)$ if it has an $a$-transition that enjoys the same property as before and each $a$-transition departing from $s$ enjoys that property, meaning that the formula is satisfied by $s$ no matter how internal nondeterminism is resolved. We shall see that both universally interpreted variants of the logic lead to the same equivalence as the one characterized by the original interpretation of the original PML. Indeed, $\sim_{\mathrm{PB}, \mathrm{gbg}, \leq}$ has also many other characterizations (see App. A), and this leads us to the convincement that it is an interesting behavioral relation for nondeterministic and probabilistic processes.

The rest of the paper is organized as follows. In Sect. 2, we provide the necessary background. The interpretations of PML over the non-alternating model are introduced in Sect. 3 and the new probabilistic bisimilarities that they characterize are presented in Sect. 4. Finally, Sect. 5 draws some conclusions. All proofs of our results are collected in App. B.

\section{Background}

In this section, we define a model for nondeterministic and probabilistic processes. Then, we recast in this general model the bisimilarity in [12] and the probabilistic bimilarity in [16], together with their HML and PML characterizations. Finally, we recall the probabilistic bisimilarity in [22] and its modal logic characterization for both the non-alternating case and the alternating case.

\subsection{The NPLTS Model}

Processes combining nondeterminism and probability are typically described by means of extensions of the LTS model, in which every action-labeled transition goes from a source state to a probability distribution over target states rather than to a single target state. The resulting processes are essentially Markov decision processes [8] and are representative of a number of slightly different probabilistic computational models including internal nondeterminism such as, e.g., concurrent Markov chains [28], strictly alternating models [10], probabilistic automata in the sense of [21], and the denotational probabilistic models in [14] (see [24] for an overview). We formalize them as a variant of simple probabilistic automata [21].

Definition 1. A nondeterministic and probabilistic labeled transition system, NPLTS for short, is a triple $(S, A, \longrightarrow)$ where:

- $S$ is an at most countable set of states.

- A is a countable set of transition-labeling actions.

$-\longrightarrow \subseteq S \times A \times \operatorname{Distr}(S)$ is a transition relation, where $\operatorname{Distr}(S)$ is the set of probability distributions over $S$. 
A transition $(s, a, \mathcal{D})$ is written $s \stackrel{a}{\longrightarrow} \mathcal{D}$. We say that $s^{\prime} \in S$ is not reachable from $s$ via that $a$-transition if $\mathcal{D}\left(s^{\prime}\right)=0$, otherwise we say that it is reachable with probability $p=\mathcal{D}\left(s^{\prime}\right)$. The reachable states form the support of $\mathcal{D}$, i.e., $\operatorname{supp}(\mathcal{D})=\left\{s^{\prime} \in S \mid \mathcal{D}\left(s^{\prime}\right)>0\right\}$. We write $s \stackrel{a}{\longrightarrow}$ to indicate that $s$ has an $a$-transition. The choice among all the transitions departing from $s$ is external and nondeterministic, while the choice of the target state for a specific transition is internal and probabilistic.

The notion of NPLTS yields a non-alternating model [21] and embeds the following restricted models:

- Fully nondeterministic processes: every transition is Dirac, i.e., it leads to a distribution that concentrates all the probability mass into one target state.

- Fully probabilistic processes: every state has at most one outgoing transition.

- Reactive probabilistic processes: no state has two or more outgoing transitions labeled with the same action [27]. These processes include the probabilistic automata in the sense of [20].

- Alternating processes: every state that enables a non-Dirac transition enables only that transition. Similar to $[29,19]$, these processes consist of a non-strict alternation of fully nondeterministic states and fully probabilistic states, with the addition that transitions departing from fully probabilistic states are labeled with actions.

An NPLTS can be depicted as a directed graph-like structure in which vertices represent states and action-labeled edges represent action-labeled transitions. Given a transition $s \stackrel{a}{\longrightarrow} \mathcal{D}$, the corresponding $a$-labeled edge goes from the vertex representing state $s$ to a set of vertices linked by a dashed line, each of which represents a state $s^{\prime} \in \operatorname{supp}(\mathcal{D})$ and is labeled with $\mathcal{D}\left(s^{\prime}\right)$ - label omitted if $\mathcal{D}\left(s^{\prime}\right)=1$. Four NPLTS models are shown in Figs. 1 and 2 .

We say that an NPLTS $(S, A, \longrightarrow)$ is image finite iff for all $s \in S$ and $a \in A$ the set $\{\mathcal{D} \in \operatorname{Distr}(S) \mid s \stackrel{a}{\longrightarrow} \mathcal{D}\}$ is finite. Following [16], we say that it satisfies the minimal probability assumption iff there exists $\epsilon \in \mathbb{R}_{>0}$ such that, whenever $s \stackrel{a}{\longrightarrow} \mathcal{D}$, then for all $s^{\prime} \in S$ either $\mathcal{D}\left(s^{\prime}\right)=0$ or $\mathcal{D}\left(s^{\prime}\right) \geq \epsilon$; this implies that $\operatorname{supp}(\mathcal{D})$ is finite because it can have at most $\lceil 1 / \epsilon\rceil$ elements. If $\mathcal{D}\left(s^{\prime}\right)$ is a multiple of $\epsilon$ for all $s^{\prime} \in S$, then the minimal deviation assumption is also satisfied.

Sometimes, instead of ordinary transitions, we will consider combined transitions [22], each being a convex combination of equally labeled transitions. Given an NPLTS $(S, A, \longrightarrow), s \in S, a \in A$, and $\mathcal{D} \in \operatorname{Distr}(S)$, in the following we write $s \stackrel{a}{\longrightarrow} \mathcal{D}$ iff there exist $n \in \mathbb{N}_{>0},\left\{p_{i} \in \mathbb{R}_{0,1]} \mid 1 \leq i \leq n\right\}$, and $\left\{s \stackrel{a}{\longrightarrow} \mathcal{D}_{i} \mid 1 \leq i \leq n\right\}$ such that $\sum_{i=1}^{n} p_{i}=1$ and $\sum_{i=1}^{n} p_{i} \cdot \mathcal{D}_{i}=\mathcal{D}$.

\subsection{Bisimilarity for Fully Nondeterministic Processes}

We recast in the NPLTS model the definition of bisimilarity for fully nondeterministic processes in [12]. In this case, the target of each transition is a Dirac distribution $\delta_{s}$ for $s \in S$, i.e., $\delta_{s}(s)=1$ and $\delta_{s}\left(s^{\prime}\right)=0$ for all $s^{\prime} \in S \backslash\{s\}$. 
Definition 2. Let $(S, A, \longrightarrow)$ be an NPLTS in which the target of each transition is a Dirac distribution. A relation $\mathcal{B}$ over $S$ is a bisimulation iff, whenever $\left(s_{1}, s_{2}\right) \in \mathcal{B}$, then for all actions $a \in A$ it holds that:

- For each $s_{1} \stackrel{a}{\longrightarrow} \delta_{s_{1}^{\prime}}$ there exists $s_{2} \stackrel{a}{\longrightarrow} \delta_{s_{2}^{\prime}}$ such that $\left(s_{1}^{\prime}, s_{2}^{\prime}\right) \in \mathcal{B}$.

- For each $s_{2} \stackrel{a}{\longrightarrow} \delta_{s_{2}^{\prime}}$ there exists $s_{1} \stackrel{a}{\longrightarrow} \delta_{s_{1}^{\prime}}$ such that $\left(s_{1}^{\prime}, s_{2}^{\prime}\right) \in \mathcal{B}$.

We denote by $\sim_{\mathrm{B}}$ the largest bisimulation.

Given an image-finite NPLTS $(S, A, \longrightarrow)$ in which the target of each transition is a Dirac distribution, the relation $\sim_{B}$ is characterized by the so-called Hennessy-Milner logic (HML) [12]. The set $\mathbb{F}_{\text {HML }}$ of its formulae is generated by the following grammar $(a \in A)$ :

$$
\phi::=\text { true }|\neg \phi| \phi \wedge \phi \mid\langle a\rangle \phi
$$

The semantics of HML can be defined through an interpretation function $\mathcal{M}_{\mathrm{HML}}$ that associates with any formula in $\mathbb{F}_{\mathrm{HML}}$ the set of states satisfying the formula:

$$
\begin{aligned}
\mathcal{M}_{\mathrm{HML}} \llbracket \text { true } \rrbracket & =S \\
\mathcal{M}_{\mathrm{HML}} \llbracket \neg \phi \rrbracket & =S \backslash \mathcal{M}_{\mathrm{HML}} \llbracket \phi \rrbracket \\
\mathcal{M}_{\mathrm{HML}} \llbracket \phi_{1} \wedge \phi_{2} \rrbracket & =\mathcal{M}_{\mathrm{HML}} \llbracket \phi_{1} \rrbracket \cap \mathcal{M}_{\mathrm{HML}} \llbracket \phi_{2} \rrbracket \\
\mathcal{M}_{\mathrm{HML}} \llbracket\langle a\rangle \phi \rrbracket & =\left\{s \in S \mid \exists s^{\prime} \in \mathcal{M}_{\mathrm{HML}} \llbracket \phi \rrbracket . s \stackrel{a}{\longrightarrow} \delta_{s^{\prime}}\right\}
\end{aligned}
$$

\subsection{Bisimilarity for Reactive Probabilistic Processes}

We recast in the NPLTS model also the definition of probabilistic bisimilarity for reactive probabilistic processes in [16]. In the following, we let $\mathcal{D}\left(S^{\prime}\right)=$ $\sum_{s^{\prime} \in S^{\prime}} \mathcal{D}\left(s^{\prime}\right)$ for $\mathcal{D} \in \operatorname{Distr}(S)$ and $S^{\prime} \subseteq S$.

Definition 3. Let $(S, A, \longrightarrow)$ be an NPLTS in which the transitions of each state have different labels. An equivalence relation $\mathcal{B}$ over $S$ is a probabilistic bisimulation iff, whenever $\left(s_{1}, s_{2}\right) \in \mathcal{B}$, then for all actions $a \in A$ and equivalence classes $C \in S / \mathcal{B}$ it holds that for each $s_{1} \stackrel{a}{\longrightarrow} \mathcal{D}_{1}$ there exists $s_{2} \stackrel{a}{\longrightarrow} \mathcal{D}_{2}$ such that $\mathcal{D}_{1}(C)=\mathcal{D}_{2}(C)$. We denote by $\sim_{\mathrm{PB}}$ the largest probabilistic bisimulation.

Given an NPLTS $(S, A, \longrightarrow)$ satisfying the minimal deviation assumption in which the transitions of each state have different labels, the relation $\sim_{\mathrm{PB}}$ is characterized by PML $[16,17]$. The set $\mathbb{F}_{\mathrm{PML}}$ of its formulae is generated by the following grammar $\left(a \in A, p \in \mathbb{R}_{[0,1]}\right)$ :

$$
\phi::=\text { true }|\neg \phi| \phi \wedge \phi \mid\langle a\rangle_{p} \phi
$$

The semantics of PML can be defined through an interpretation function $\mathcal{M}_{\mathrm{PML}}$ that differs from $\mathcal{M}_{\mathrm{HML}}$ only for the last clause, which becomes as follows:

$\mathcal{M}_{\mathrm{PML}} \llbracket\langle a\rangle_{p} \phi \rrbracket=\left\{s \in S \mid \exists \mathcal{D} \in \operatorname{Distr}(S) . s \stackrel{a}{\longrightarrow} \mathcal{D} \wedge \mathcal{D}\left(\mathcal{M}_{\mathrm{PML}} \llbracket \phi \rrbracket\right) \geq p\right\}$ Note that, in this reactive setting, if an $a$-labeled transition exists that goes from $s$ to $\mathcal{D}$, then it is the only $a$-labeled transition departing from $s$, and hence $\mathcal{D}$ is unique. In [9], it was subsequently shown that probabilistic bisimilarity for reactive probabilistic processes can be characterized by PML without negation and that the existence of neither a minimal deviation nor a minimal probability needs to be assumed to achieve the characterization result. 


\subsection{Bisimilarity for Non-Alternating and Alternating Processes}

For NPLTS models in their full generality, we now recall two probabilistic bisimulation equivalences defined in [22]. Both of them check whether the probabilities of all classes of equivalent states - i.e., the class distributions - reached by the two transitions considered in the bisimulation game are equal.

The first equivalence relies on deterministic schedulers for resolving nondeterminism. This means that, when responding to an $a$-transition of the challenger, the defender can only select a single $a$-transition (if any).

Definition 4. Let $(S, A, \longrightarrow)$ be an NPLTS. An equivalence relation $\mathcal{B}$ over $S$ is a class-distribution probabilistic bisimulation iff, whenever $\left(s_{1}, s_{2}\right) \in \mathcal{B}$, then for all actions $a \in A$ it holds that for each $s_{1} \stackrel{a}{\longrightarrow} \mathcal{D}_{1}$ there exists $s_{2} \stackrel{a}{\longrightarrow} \mathcal{D}_{2}$ such that, for all equivalence classes $C \in S / \mathcal{B}, \mathcal{D}_{1}(C)=\mathcal{D}_{2}(C)$. We denote by $\sim_{\mathrm{PB} \text {,dis }}$ the largest class-distribution probabilistic bisimulation.

While in Def. 3 the quantification over $C \in S / \mathcal{B}$ can be placed before or after the transitions because $s_{1}$ and $s_{2}$ can have at most one outgoing $a$-transition each, in Def. 4 it is important for the quantification to be after the transitions.

The second equivalence relies instead on randomized schedulers. This means that, when responding to an $a$-transition of the challenger, the defender can select a convex combination of $a$-transitions (if any). In the following, the acronym ct stands for "based on combined transitions".

Definition 5. Let $(S, A, \longrightarrow)$ be an NPLTS. An equivalence relation $\mathcal{B}$ over $S$ is a class-distribution ct-probabilistic bisimulation iff, whenever $\left(s_{1}, s_{2}\right) \in \mathcal{B}$, then for all actions $a \in A$ it holds that for each $s_{1} \stackrel{a}{\longrightarrow} \mathcal{D}_{1}$ there exists $s_{2} \stackrel{a}{\longrightarrow}{ }_{\mathrm{c}} \mathcal{D}_{2}$ such that, for all equivalence classes $C \in S / \mathcal{B}, \mathcal{D}_{1}(C)=\mathcal{D}_{2}(C)$. We denote by $\sim_{\mathrm{PB}, \mathrm{dis}}^{\mathrm{ct}}$ the largest class-distribution ct-probabilistic bisimulation.

In order to obtain a modal logic characterization for $\sim_{\mathrm{PB}, \mathrm{dis}}$ and $\sim_{\mathrm{PB}, \mathrm{dis}}^{\mathrm{ct}}$, in $[18,13]$ an extension of HML much richer than PML was defined. The main differences are that (i) formulae are interpreted over probability distribution on states rather than over single states and (ii) the modal operator $\langle a\rangle_{p}$. is split into the original modal operator $\langle a\rangle$. of HML and an additional unary operator $[\cdot]_{p}$ such that state distribution $\mathcal{D}$ satisfies $[\phi]_{p}$ if $\mathcal{D}$ associates with the set of states satisfying $\phi$ a probability that is at least $p$.

In [11], the same equivalences (lifted to state distributions) were differently characterized by adding to HML a binary operator $\cdot \oplus_{p} \cdot$, where $\phi_{1} \oplus_{p} \phi_{2}$ asserts decomposability of a state distribution to satisfy the two subformulae.

For alternating processes, i.e., NPLTS models in which every state that enables a non-Dirac transition enables only that transition, the following holds:

$-\sim_{\mathrm{PB}, \mathrm{dis}}$ and $\sim_{\mathrm{PB} \text {,dis }}^{\mathrm{ct}}$ collapse into a single equivalence that coincides with those defined in $[10,19]$ for alternating processes, as shown in [23].

$-\sim_{\mathrm{PB}, \mathrm{dis}}$ is again characterized by the original PML, as shown in [18]. 


\section{Interpreting PML over NPLTS Models}

PML was originally interpreted in $[16,17]$ on reactive probabilistic processes and then in [18] on alternating processes. The same interpretation can be applied to general NPLTS models by establishing that state $s$ satisfies formula $\langle a\rangle_{p} \phi$ iff there exists a resolution of internal nondeterminism such that $s$ can perform an $a$-transition and afterwards reaches with probability at least $p$ a set of states that satisfy $\phi$. This existential interpretation only provides a weak guarantee of fulfilling properties, as it depends on how internal nondeterminism is resolved.

A different interpretation can be adopted by following [3]: $s$ satisfies $\langle a\rangle_{p} \phi$ iff, for each resolution of internal nondeterminism, $s$ can perform an $a$-transition and afterwards reaches with probability at least $p$ a set of states that satisfy $\phi$. The resulting universal interpretation provides a strong guarantee of fulfilling properties because, no matter how internal nondeterminism is resolved, a certain behavior is ensured.

We denote by $\mathrm{PML}_{\exists,>}$ and $\mathrm{PML}_{\forall,>}$ the logics resulting from the two different interpretations of the diamond operator, which we formalize as follows:

$$
\begin{aligned}
& \mathcal{M}_{\mathrm{PML}_{\exists, \geq}} \llbracket\langle a\rangle_{p} \phi \rrbracket=\left\{s \in S \mid \exists \mathcal{D} . s \stackrel{a}{\longrightarrow} \mathcal{D} \wedge \mathcal{D}\left(\mathcal{M}_{\mathrm{PML}_{\exists, \geq}} \llbracket \phi \rrbracket\right) \geq p\right\} \\
& \mathcal{M}_{\mathrm{PML}_{\forall, \geq}} \llbracket\langle a\rangle_{p} \phi \rrbracket=\left\{s \in S \mid s \stackrel{a}{\longrightarrow} \wedge \forall \mathcal{D} . s \stackrel{a}{\longrightarrow} \mathcal{D} \Longrightarrow \mathcal{D}\left(\mathcal{M}_{\mathrm{PML}_{\forall, \geq}} \llbracket \phi \rrbracket\right) \geq p\right\}
\end{aligned}
$$

Finally, we denote by $\mathrm{PML}_{\exists, \mathrm{I}}$ and $\mathrm{PML}_{\forall, \mathrm{I}}$ two further variants generalizing the previous two logics, in which the probability value $p$ is replaced by a probability interval $\left[p_{1}, p_{2}\right]$ - where $p_{1}, p_{2} \in \mathbb{R}_{[0,1]}$ are such that $p_{1} \leq p_{2}-$ and the resulting diamond operator is interpreted as follows:

$\mathcal{M}_{\mathrm{PML}_{\exists, \mathrm{I}}} \llbracket\langle a\rangle_{\left[p_{1}, p_{2}\right]} \phi \rrbracket=\left\{s \in S \mid \exists \mathcal{D} . s \stackrel{a}{\longrightarrow} \mathcal{D} \wedge p_{1} \leq \mathcal{D}\left(\mathcal{M}_{\mathrm{PML}_{\exists, \mathrm{I}}} \llbracket \phi \rrbracket\right) \leq p_{2}\right\}$

$\mathcal{M}_{\mathrm{PML}_{\forall, \mathrm{I}}} \llbracket\langle a\rangle_{\left[p_{1}, p_{2}\right]} \phi \rrbracket=\left\{s \in S \mid s \stackrel{a}{\longrightarrow} \wedge \forall \mathcal{D} . s \stackrel{a}{\longrightarrow} \mathcal{D} \Longrightarrow p_{1} \leq \mathcal{D}\left(\mathcal{M}_{\mathrm{PML}_{\forall, \mathrm{I}}} \llbracket \phi \rrbracket\right) \leq p_{2}\right\}$ Note that $\langle a\rangle_{p} \phi$ can be encoded as $\langle a\rangle_{[p, 1]} \phi$ because $p$ is a lower bound.

In the following, if $\mathrm{L}$ is one of the above variants of PML, then we denote by $\mathcal{F}_{\mathrm{L}}(s)$ the set of formulae in $\mathbb{F}_{\mathrm{L}}$ satisfied by state $s$ and we let $s_{1} \sim_{\mathrm{L}} s_{2}$ iff $\mathcal{F}_{\mathrm{L}}\left(s_{1}\right)=\mathcal{F}_{\mathrm{L}}\left(s_{2}\right)$. Interestingly enough, the equivalences induced by the universally interpreted variants are the same and coincide with the equivalence induced by the existentially interpreted variant with probabilistic bound. In contrast, the equivalence induced by $\mathrm{PML}_{\exists, \mathrm{I}}$ is finer (see App. A).

\section{Bisimilarities Characterized by PML}

In this section, we introduce the probabilistic bisimilarities for NPLTS models that are characterized by PML as interpreted in the previous section. Before presenting their definition, we highlight the differences with respect to $\sim_{\mathrm{PB} \text {,dis }}$.

Firstly, instead of comparing the probability distributions over all classes of equivalent states reached by the transitions considered in the bisimulation game, the new equivalences focus on a single equivalence class at a time. Therefore, similar to [26], given an action $a$ the probability distribution over all classes of equivalent states reached by an $a$-transition of the challenger can now be matched by means of several (not just by one) $a$-transitions of the defender, each taking care of a different class. 
Secondly, the new equivalences take into account the probability of reaching groups of equivalence classes rather than individual classes. This would make no difference in the case of $\sim_{\mathrm{PB} \text {,dis }}$, while here it significantly changes the discriminating power (see App. A). Due to the previous and the current difference with respect to $\sim_{\mathrm{PB} \text {,dis }}$, we call these equivalences group-by-group probabilistic bisimilarities.

Thirdly, the new equivalences come in several variants depending on whether, in the bisimulation game, the probabilities of reaching a certain group of classes of equivalent states are compared based on $=$ or $\leq$. Again, this would make no difference in the case of $\sim_{\mathrm{PB} \text {,dis }}$.

In the following, we let $\bigcup \mathcal{G}=\bigcup_{C \in \mathcal{G}} C$ when $\mathcal{G} \in 2^{S / \mathcal{B}}$ is a group of equivalence classes with respect to an equivalence relation $\mathcal{B}$ over $S$.

Definition 6. Let $(S, A, \longrightarrow)$ be an NPLTS and the relational operator $\bowtie \in$ $\{=, \leq\}$. An equivalence relation $\mathcal{B}$ over $S$ is a $\bowtie$-group-by-group probabilistic bisimulation iff, whenever $\left(s_{1}, s_{2}\right) \in \mathcal{B}$, then for all actions $a \in A$ and groups of equivalence classes $\mathcal{G} \in 2^{S / \mathcal{B}}$ it holds that for each $s_{1} \stackrel{a}{\longrightarrow} \mathcal{D}_{1}$ there exists $s_{2} \stackrel{a}{\longrightarrow} \mathcal{D}_{2}$ such that $\mathcal{D}_{1}(\bigcup \mathcal{G}) \bowtie \mathcal{D}_{2}(\bigcup \mathcal{G})$. We denote by $\sim_{\mathrm{PB}, \mathrm{gbg}, \bowtie}$ the largest $\bowtie$-group-by-group probabilistic bisimulation.

The definition of $\sim_{\mathrm{PB}, \mathrm{gbg}, \bowtie}$ assumes the use of deterministic schedulers, but it can be easily extended to the case of randomized schedulers by analogy with $\sim_{\mathrm{PB}, \mathrm{dis}}^{\mathrm{ct}}$, thus yielding $\sim_{\mathrm{PB}, \mathrm{gbg}, \bowtie}^{\mathrm{ct}}$.

Note that, while in Def. 4 the quantification over $C \in S / \mathcal{B}$ is after the transitions, in Def. 6 the quantification over $\mathcal{G} \in 2^{S / \mathcal{B}}$ is before the transitions thus allowing a transition of the challenger to be matched by several transitions of the defender depending on the target groups.

The relation $\sim_{\mathrm{PB}, \mathrm{gbg},=}$ identifies the two systems in Fig. 1, whilst the relation $\sim_{\mathrm{PB}, \mathrm{gbg}, \leq} \leq$ also identifies the two systems in Fig. 2 . The following theorem shows that $\sim_{\mathrm{PB}, \mathrm{dis}}$ is finer than $\sim_{\mathrm{PB}, \mathrm{gbg},=}$ and that the latter is finer than $\sim_{\mathrm{PB}, \mathrm{gbg}, \leq}$.

Theorem 1. Let $(S, A, \longrightarrow)$ be an NPLTS and $s_{1}, s_{2} \in S$. Then:

1. $s_{1} \sim_{\mathrm{PB}, \mathrm{dis}} s_{2} \Longrightarrow s_{1} \sim_{\mathrm{PB}, \mathrm{gbg},=} s_{2}$.

2. $s_{1} \sim \mathrm{PB}, \mathrm{gbg},=s_{2} \Longrightarrow s_{1} \sim \mathrm{PB}, \mathrm{gbg}, \leq s_{2}$.

The two implications above cannot be reversed: Fig. 1 shows that $\sim_{\mathrm{PB}, \mathrm{dis}}$ is strictly finer than $\sim_{\mathrm{PB}, \mathrm{gbg},=}$ and Fig. 2 shows that $\sim_{\mathrm{PB}, \mathrm{gbg},=}$ is strictly finer than $\sim_{\mathrm{PB}, \mathrm{gbg}, \leq}$.

In [22], it is also shown that $\sim_{\mathrm{PB}, \mathrm{dis}}^{\mathrm{ct}}$, the variant of $\sim_{\mathrm{PB}, \mathrm{dis}}$ that relies on randomized schedulers (see Def. 5 ), is strictly finer than $\sim_{\mathrm{PB} \text {,dis }}$. On the contrary, we have that $\sim_{\mathrm{PB}, \text { gbg }} \leq$ coincides with its ct-variant, and hence it is insensitive to the choice between deterministic or randomized schedulers used to resolve nondeterminism. This is not the case for $\sim_{\mathrm{PB}, \mathrm{gbg},=}$. Indeed, the ct-variants of $\sim_{\mathrm{PB}, \mathrm{gbg},=}$ coincides with $\sim_{\mathrm{PB}, \mathrm{gbg}, \leq}$, meaning that, in the bisimulation game, randomized schedulers reduce the discriminating power of the $=$-comparison of probabilities to that of the $\leq$-comparison. As expected, the ct-variant of $\sim_{\mathrm{PB} \text {,dis }}$ 


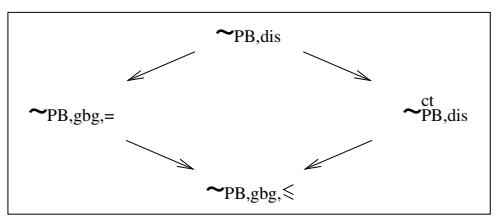

Fig. 3. Relating group-by-group and distribution-based probabilistic bisimilarities

is coarser than that of $\sim_{\mathrm{PB}, \mathrm{gbg},=}$, and thus also coarser than $\sim_{\mathrm{PB}, \mathrm{gbg}, \leq}$ and $\sim_{\mathrm{PB}, \mathrm{gbg}, \leq}^{\mathrm{ct}}$

Theorem 2. Let $\mathcal{U}=(S, A, \longrightarrow)$ be an NPLTS and $s_{1}, s_{2} \in S$. Then:

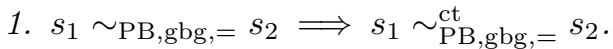

2. $s_{1} \sim \sim_{\mathrm{PB}, \mathrm{dis}}^{\mathrm{ct}} s_{2} \Longrightarrow s_{1} \sim{ }_{\mathrm{PB}, \mathrm{gbg},=}^{\mathrm{ct}}=s_{2}$.

3. $s_{1} \sim_{\mathrm{PB}, \mathrm{gbg}, \leq} s_{2} \Longleftrightarrow s_{1} \sim_{\mathrm{PB}, \mathrm{gbg}, \leq}^{\mathrm{ct}} s_{2} \Longleftrightarrow s_{1} \sim_{\mathrm{PB}, \mathrm{gbg},=}^{\mathrm{ct}} s_{2}$ when $\mathcal{U}$ is image-finite.

The inclusions of $\sim_{\mathrm{PB}, \mathrm{gbg},=}$ in $\sim_{\mathrm{PB}, \mathrm{gbg},=}^{\mathrm{ct}}$ is strict. This can be proved by using the systems in Fig. 2. Indeed, $s_{1} \chi_{\mathrm{PB}, \mathrm{gbg},=}=s_{2}$ while $s_{1} \sim_{\mathrm{PB}, \mathrm{gbg},=}^{\mathrm{ct}} s_{2}$; the latter holds because the central offer-transition of $s_{1}$ can be matched by a convex combination of the two offer-transitions of $s_{2}$ both weighted by 0.5. Also the inclusion of $\sim_{\mathrm{PB} \text {, dis }}^{\mathrm{ct}}$ in $\sim_{\mathrm{PB}, \mathrm{gbg}}, \leq$ is strict. This is evidenced by the systems in Fig. 1; no transition of $s_{1}$ can be obtained as the convex combination of transitions of $s_{2}$ and thus $s_{1} \chi_{\mathrm{PB}, \mathrm{dis}}^{\mathrm{ct}} s_{2}$. Finally, it also holds that $\sim_{\mathrm{PB}, \mathrm{dis}}^{\mathrm{ct}}$ and $\sim_{\mathrm{PB}, \mathrm{gbg},=}$ are incomparable. Indeed, the two systems in Fig. 1 are equated by $\sim_{\mathrm{PB}, \mathrm{gbg}},=$ and distinguished by $\sim_{\mathrm{PB}, \mathrm{dis}}^{\mathrm{ct}}$, while the two systems in Fig. 2 are distinguished by $\sim_{\mathrm{PB}, \mathrm{gbg},=}$ and equated by $\sim_{\mathrm{PB}, \mathrm{dis}}^{\mathrm{ct}}$. These results are summarized in Fig. 3.

For the new probabilistic bisimilarities, different alternative definitions can be obtained by varying the requirements on the comparison between sets of probabilities by considering not only $=$ and $\leq$ but also $\geq$, or by comparing only extremal probabilities $(\sqcup$ and/or $\sqcap$ ). Quite surprisingly, all relations but the one based on $=$ do collapse. Due to lack of space, we do not consider these variants in the present paper (see App. A).

Before moving to the modal logic characterization results, we show that the two group-by-group probabilistic bisimilarities and their ct-variants collapse on existing bisimilarities when one considers NPLTS models with a restricted interplay between probabilistic and non-determinism. In particular they coincide with: (i) the bisimilarity in [12] for fully nondeterministic processes (see Def. 2); (ii) the probabilistic bisimilarity in [16] for reactive probabilistic processes (see Def. 3); (iii) the probabilistic bisimilarities in [22] when alternating processes are considered. These results provide additional evidences that PML can be a uniform framework for reasoning on different classes of processes including probability and various degrees of nondeterminism. 
Theorem 3. Let $(S, A, \longrightarrow)$ be an NPLTS in which the target of each transition is a Dirac distribution. Let $s_{1}, s_{2} \in S$ and $\bowtie \in\{=, \leq\}$. Then:

$$
s_{1} \sim_{\mathrm{PB}, \mathrm{gbg}, \bowtie} s_{2} \Longleftrightarrow s_{1} \sim_{\mathrm{PB}, \mathrm{gbg}, \bowtie}^{\mathrm{ct}} s_{2} \Longleftrightarrow s_{1} \sim_{\mathrm{B}} s_{2}
$$

Theorem 4. Let $(S, A, \longrightarrow)$ be an NPLTS in which the transitions of each state have different labels. Let $s_{1}, s_{2} \in S$ and $\bowtie \in\{=, \leq\}$. Then:

$$
s_{1} \sim_{\mathrm{PB}, \mathrm{gbg}, \bowtie} s_{2} \Longleftrightarrow s_{1} \sim_{\mathrm{PB}, \mathrm{gbg}, \bowtie}^{\mathrm{ct}} s_{2} \Longleftrightarrow s_{1} \sim_{\mathrm{PB}} s_{2}
$$

Theorem 5. Let $(S, A, \longrightarrow)$ be an NPLTS in which every state that enables a non-Dirac transition enables only that transition. If $s_{1}, s_{2} \in S$ and $\bowtie \in\{=, \leq\}$ then:

$$
\begin{aligned}
& s_{1} \sim_{\mathrm{PB}, \mathrm{gbg}, \bowtie} s_{2} \Longleftrightarrow s_{1} \sim_{\mathrm{PB}, \text { dis }} s_{2} \\
& s_{1} \sim_{\mathrm{PB}, \mathrm{gbg}, \bowtie}^{\text {ct }} s_{2} \Longleftrightarrow s_{1} \sim_{\mathrm{PB}, \mathrm{dis}}^{\mathrm{ct}} s_{2}
\end{aligned}
$$

We are now ready to establish our logical characterization results and to show that $\sim_{\mathrm{PB}, \mathrm{gbg},=}$ is characterized by $\mathrm{PML}_{\exists, \mathrm{I}}$ while $\sim_{\mathrm{PB}, \mathrm{gbg}, \leq}$ is characterized by $\mathrm{PML}_{\exists, \geq}$, under the image finiteness and minimal probability assumptions.

Theorem 6. Let $(S, A, \longrightarrow)$ be an image-finite NPLTS satisfying the minimal probability assumption. Let $s_{1}, s_{2} \in S$. Then:

$$
s_{1} \sim_{\mathrm{PB}, \mathrm{gbg},=} s_{2} \Longleftrightarrow s_{1} \sim_{\mathrm{PML}_{\exists, \mathrm{I}}} s_{2}
$$

Theorem 7. Let $(S, A, \longrightarrow)$ be an image-finite NPLTS satisfying the minimal probability assumption. Let $s_{1}, s_{2} \in S$. Then:

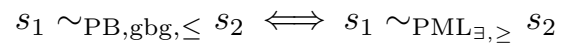

Given the importance of these results in the economy of the paper, below we sketch the proof of Thm. 6; the one for Thm. 7 follows the same pattern. First, we need to provide an alternative characterization of $\sim_{\mathrm{PB}, \mathrm{gbg},}=$ as the limit of a sequence of equivalence relations $\sim_{\mathrm{PB}, \mathrm{gbg},=}^{i}$.

For an $\operatorname{NPLTS}(S, A, \longrightarrow)$, the family $\left\{\sim_{\mathrm{PB}, \mathrm{gbg},=\mid}^{i} \mid i \in \mathbb{N}\right\}$ of equivalence relations over $S$ is inductively defined as follows:

$-\sim_{\mathrm{PB}, \mathrm{gbg},=}^{0}=S \times S$.

$-\sim_{\mathrm{PB}, \mathrm{gbg},=}^{i+1}$ is the set of all pairs $\left(s_{1}, s_{2}\right) \in \sim_{\mathrm{PB}, \mathrm{gbg},=}^{i}$ such that for all actions

$a \in A$ and groups of equivalence classes $\mathcal{G} \in 2^{S / \sim_{\mathrm{PB}, \mathrm{gbg}}^{i}=}$ it holds that for each $s_{1} \stackrel{a}{\longrightarrow} \mathcal{D}_{1}$ there exists $s_{2} \stackrel{a}{\longrightarrow} \mathcal{D}_{2}$ such that $\mathcal{D}_{1}(\bigcup \mathcal{G})=\mathcal{D}_{2}(\bigcup \mathcal{G})$.

Each equivalence relation $\sim_{\mathrm{PB}, \mathrm{gbg},=}^{i}$ identifies those states that cannot be distinguished within $i$ steps of computation. The following lemma guarantees that two states of an image-finite NPLTS are equivalent according to $\sim_{\mathrm{PB}, \mathrm{gbg},=}$ iff they are equivalent according to all the relations $\sim_{\mathrm{PB}, \mathrm{gbg},=}^{i}$.

Lemma 1. Let $(S, A, \longrightarrow)$ be an image-finite NPLTS. Then:

$$
\sim_{\mathrm{PB}, \mathrm{gbg},=}=\bigcap_{i \in \mathbb{N}} \sim_{\mathrm{PB}, \mathrm{gbg},=}^{i}
$$


The second step of the proof is to show that two states are equated by $\sim_{\mathrm{PB}, \mathrm{gbg},=}^{i}$ iff they satisfy the same formulae in $\mathbb{F}_{\mathrm{PML}}^{i}$, which is the set of formulae in $\mathbb{F}_{\mathrm{PML} \exists, \mathrm{I}}$ whose maximum number of nested diamond operators is at most $i$.

Lemma 2. Let $(S, A, \longrightarrow)$ be an image-finite NPLTS satisfying the minimal probability assumption. Let $s_{1}, s_{2} \in S$. Then for all $i \in \mathbb{N}$ :

$$
s_{1} \sim_{\mathrm{PB}, \mathrm{gbg},=}^{i} s_{2} \Longleftrightarrow \mathcal{F}_{\mathrm{PML} \exists, \mathrm{I}}^{i}\left(s_{1}\right)=\mathcal{F}_{\mathrm{PML} \exists, \mathrm{I}}^{i}\left(s_{2}\right)
$$

Now Thm. 6 directly follows from Lemma 1 and Lemma 2. The same result would not hold if $\mathrm{PML}_{\exists, \geq}$ was used. For instance, the two states $s_{1}$ and $s_{2}$ in Fig. 2, which are not related by $\sim_{\mathrm{PB}, \mathrm{gbg},=}$ as can be seen by considering the $\mathrm{PML}_{\exists, \mathrm{I}}$ formula $\langle\text { offer }\rangle_{[0.5,0.5]}\langle\text { head }\rangle_{[1,1]}$ true, cannot be distinguished by any $\mathrm{PML}_{\exists, \geq}$ formula.

It is easy to see that $\sim_{\mathrm{PB}, \mathrm{gbg},=}^{\mathrm{ct}}$ and $\sim_{\mathrm{PB}, \mathrm{gbg}, \leq}^{\mathrm{ct}}$ are respectively characterized by $\mathrm{PML}_{\exists, \mathrm{I}}^{\mathrm{ct}}$ and $\mathrm{PML}_{\exists, \geq}^{\mathrm{ct}}$, in which the interpretation of the diamond operator relies on combined transitions instead of ordinary ones.

\section{Conclusion}

We have addressed the problem of defining behavioral relations for nondeterministic and probabilistic processes that are characterized by modal logics as close as possible to PML, the natural probabilistic version of the by now standard HML for fully nondeterministic processes. We have introduced two new probabilistic bisimilarities $\left(\sim_{\mathrm{PB}, \mathrm{gbg}, \leq}\right.$ and $\left.\sim_{\mathrm{PB}, \mathrm{gbg},=}\right)$ following a group-by-group approach and studied their relationships with an existential and a universal interpretation of two variants of PML, in which the diamond is respectively decorated with a probability lower bound and a probability interval. All the resulting logical equivalences, except the one based on existential interpretation and probability intervals, do coincide with $\sim_{\mathrm{PB}, \mathrm{gbg}, \leq} \leq$. Interestingly enough, $\sim_{\mathrm{PB}, \mathrm{gbg},=}$, which is finer than $\sim_{\mathrm{PB}, \mathrm{gbg}, \leq}$, has naturally emerged in a framework recently developed to provide a uniform model and uniformly defined behavioral equivalences for different classes of (nondeterministic, stochastic, probabilitic) processes [2].

These results, together with backward compatibility of our equivalences with those already defined for models with a restricted interplay between probability and nondeterminism, provide additional evidences that PML can be a uniform framework for reasoning on different classes of processes including probability and various degrees of nondeterminism.

We have also considered variants of our equivalences that rely on combined transitions and have proved that all such variants collapse on $\sim_{\mathrm{PB}, \mathrm{gbg}, \leq}$. This suggests that, in the group-by-group approach, resolving nondeterminism with deterministic or randomized schedulers leads to the same identifications except when checking for equality of probabilities.

Our work has some interesting points in common with [25], where new probabilistic bisimilarities over nondeterministic and probabilistic Kripke structures 
have been defined that are in full agreement with PCTL, PCTL*, and their variants without the next-time operator. Indeed, both [25] and our work witness that, in order to characterize the equivalences induced by PCTL/PCTL*/PML in a nondeterministic and probabilistic setting, it is necessary to: (1) Anticipate the quantification over the sets of equivalent states to be reached in the bisimulation game, as done in [26]; (2) Consider groups of classes of equivalent states rather than only classes; (3) Compare for equality only the extremal probabilities of reaching certain sets of states rather than all the probabilities.

It is, however, worth noting that both our equivalences differ from those of [25]. There, to define probabilistic bisimilarities a multistep and inductive approach has been used and only their strong multistep 1-depth bisimulation is strongly related to $\sim_{\mathrm{PB}, \mathrm{gbg}, \leq}$. In contrast, the general probabilistic bisimilarity of [25], obtained as the limit of the chain of n-depth bisimulations, is provably finer than both our group-by-group probabilistic bisimilarities once the appropriate model transformation from Kripke structures to NPLTS is performed.

Our results and those in [25] also show that, in the case of nondeterministic and probabilistic processes, it is not possible to define a single probabilistic bisimilarity that is characterized by both PML - as interpreted in this paper and PCTL $^{*}$ - as interpreted in [3]. Thus, for nondeterministic and probabilistic processes the situation is quite different from the case of fully nondeterministic processes, where probabilistic bisimilarity is characterized by both HML [12] and $\mathrm{CTL}^{*}$ [4], and from the case of reactive probabilistic processes, where probabilistic bisimilarity is characterized by both PML $[16,17]$ and PCTL* $[1]$.

\section{References}

1. A. Aziz, V. Singhal, F. Balarin, R. Brayton, and A. Sangiovanni-Vincentelli. It usually works: The temporal logic of stochastic systems. In Proc. CAV 1995, volume 939 of LNCS, pages 155-165. Springer, 1995.

2. M. Bernardo, R. De Nicola, and M. Loreti. A uniform framework for modeling nondeterministic, probabilistic, stochastic, or mixed processes and their behavioral equivalences. Information and Computation, 225:29-82, 2013.

3. A. Bianco and L. de Alfaro. Model checking of probabilistic and nondeterministic systems. In Proc. FSTTCS 1995, volume 1026 of LNCS, pages 499-513. Springer, 1995.

4. M. Browne, E. Clarke, and O. Grümberg. Characterizing finite Kripke structures in propositional temporal logic. Theoretical Computer Science, 59:115-131, 1988.

5. E. Clarke, E. Emerson, and A. Sistla. Automatic verification of finite-state concurrent systems using temporal logic specifications. ACM Trans. on Programming Languages and Systems, 8:244-263, 1986.

6. S. Crafa and F. Ranzato. A spectrum of behavioral relations over LTSs on probability distributions. In Proc. CONCUR 2011, volume 6901 of LNCS, pages 124-139. Springer, 2011.

7. L. de Alfaro, R. Majumdar, V. Raman, and M. Stoelinga. Game refinement relations and metrics. Logical Methods in Computer Science, 4(3:7):1-28, 2008.

8. C. Derman. Finite State Markovian Decision Processes. Academic Press, 1970. 
9. J. Desharnais, A. Edalat, and P. Panangaden. Bisimulation for labelled Markov processes. Information and Computation, 179:163-193, 2002.

10. H. Hansson and B. Jonsson. A calculus for communicating systems with time and probabilities. In Proc. RTSS 1990, pages 278-287. IEEE-CS Press, 1990.

11. M. Hennessy. Exploring probabilistic bisimulations, part I. Formal Aspects of Computing, 24:749-768, 2012.

12. M. Hennessy and R. Milner. Algebraic laws for nondeterminism and concurrency. Journal of the ACM, 32:137-162, 1985.

13. H. Hermanns, A. Parma, R. Segala, B. Wachter, and L. Zhang. Probabilistic logical characterization. Information and Computation, 209:154-172, 2011.

14. H. Jifeng, K. Seidel, and A. McIver. Probabilistic models for the guarded command language. Science of Computer Programming, 28:171-192, 1997.

15. R. Keller. Formal verification of parallel programs. Communications of the ACM, 19:371-384, 1976.

16. K. Larsen and A. Skou. Bisimulation through probabilistic testing. Information and Computation, 94:1-28, 1991.

17. K. Larsen and A. Skou. Compositional verification of probabilistic processes. In Proc. CONCUR 1992, volume 630 of LNCS, pages 456-471. Springer, 1992.

18. A. Parma and R. Segala. Logical characterizations of bisimulations for discrete probabilistic systems. In Proc. FOSSACS 2007, volume 4423 of LNCS, pages 287301. Springer, 2007.

19. A. Philippou, I. Lee, and O. Sokolsky. Weak bisimulation for probabilistic systems. In Proc. CONCUR 2000, volume 1877 of LNCS, pages 334-349. Springer, 2000.

20. M. Rabin. Probabilistic automata. Information and Control, 6:230-245, 1963.

21. R. Segala. Modeling and Verification of Randomized Distributed Real-Time Systems. PhD Thesis, 1995.

22. R. Segala and N. Lynch. Probabilistic simulations for probabilistic processes. In Proc. CONCUR 1994, volume 836 of LNCS, pages 481-496. Springer, 1994.

23. R. Segala and A. Turrini. Comparative analysis of bisimulation relations on alternating and non-alternating probabilistic models. In Proc. QEST 2005, pages 44-53. IEEE-CS Press, 2005.

24. A. Sokolova and E. de Vink. Probabilistic automata: System types, parallel composition and comparison. In Validation of Stochastic Systems, volume 2925 of LNCS, pages 1-43. Springer, 2004.

25. L. Song, L. Zhang, and J. Godskesen. Bisimulations meet PCTL equivalences for probabilistic automata. In Proc. CONCUR 2011, volume 6901 of LNCS, pages 108-123. Springer, 2011.

26. M. Tracol, J. Desharnais, and A. Zhioua. Computing distances between probabilistic automata. In Proc. QAPL 2011, volume 57 of EPTCS, pages 148-162, 2011.

27. R. van Glabbeek, S. Smolka, and B. Steffen. Reactive, generative and stratified models of probabilistic processes. Information and Computation, 121:59-80, 1995.

28. M. Vardi. Automatic verification of probabilistic concurrent finite-state programs. In Proc. FOCS 1985, pages 327-338. IEEE-CS Press, 1985.

29. W. Yi and K. Larsen. Testing probabilistic and nondeterministic processes. In Proc. PSTV 1992, pages 47-61. North-Holland, 1992. 


\section{A Variants of Group-by-Group Bisimilarities}

In this appendix, we present further motivations, alternative characterizations based on extremal probabilities and universal interpretations, relationships determined by the distinguishing power, and multistep variants for the group-bygroup probabilistic bisimilarities.

\section{A.1 Class-by-Class Probabilistic Bisimilarities}

In order to motivate the use of groups of equivalence classes in Def. 6, we now introduce class-by-class variants of $\sim_{\mathrm{PB}, \text { dis }}$ by simply anticipating the quantification over equivalence classes of target states in Def. 4 .

Definition 7. Let $(S, A, \longrightarrow)$ be an NPLTS and the relational operator $\bowtie \in$ $\{=, \leq, \geq\}$. An equivalence relation $\mathcal{B}$ over $S$ is a $\bowtie$-class-by-class probabilistic bisimulation iff, whenever $\left(s_{1}, s_{2}\right) \in \mathcal{B}$, then for all actions $a \in A$ and equivalence classes $C \in S / \mathcal{B}$ it holds that for each $s_{1} \stackrel{a}{\longrightarrow} \mathcal{D}_{1}$ there exists $s_{2} \stackrel{a}{\longrightarrow} \mathcal{D}_{2}$ such that $\mathcal{D}_{1}(C) \bowtie \mathcal{D}_{2}(C)$. We denote by $\sim_{\mathrm{PB}, \mathrm{cbc}, \bowtie}$ the largest $\bowtie$-class-by-class probabilistic bisimulation.

The relations $\sim_{\mathrm{PB}, \mathrm{cbc}, \bowtie}$ are too coarse. For example, in Fig. 4 it holds that $s_{1} \sim_{\mathrm{PB}, \mathrm{cbc},=} s_{2}$, as witnessed by the equivalence relation that pairs states with identically labeled transitions. However, after performing $a$, from $s_{2}$ it is always possible to reach a state in which $c^{\prime}$ or $c^{\prime \prime}$ is enabled, whereas this is not the case from $s_{1}$.

From a modal logic perspective, none of the relations $\sim_{\mathrm{PB}, \mathrm{cbc}, \bowtie}$ is characterized by the PML variants of Sect. 3. For instance, in Fig. 4 it holds that only $s_{1}$ satisfies the following existentially interpreted formulae:

$$
\begin{aligned}
& \mathrm{PML}_{\exists, \geq}:\langle a\rangle_{0.5}\left(\left\langle c^{\prime}\right\rangle_{1} \text { true } \vee\left\langle c^{\prime \prime}\right\rangle_{1} \text { true }\right) \\
& \mathrm{PML}_{\exists, \leq}:\langle a\rangle_{0}\left(\left\langle c^{\prime}\right\rangle_{1} \text { true } \vee\left\langle c^{\prime \prime}\right\rangle_{1} \text { true }\right) \\
& \mathrm{PML}_{\exists, \mathrm{I}}: \quad \neg\langle a\rangle_{[0.2,0.3]}\left(\left\langle c^{\prime}\right\rangle_{[1,1]} \text { true } \vee\left\langle c^{\prime \prime}\right\rangle_{[1,1]} \text { true }\right)
\end{aligned}
$$

while only $s_{2}$ satisfies the following universally interpreted formulae:

$$
\begin{aligned}
& \mathrm{PML}_{\forall, \geq}:\langle a\rangle_{0.7}\left(\langle b\rangle_{1} \text { true } \vee\langle d\rangle_{1} \text { true }\right) \\
& \mathrm{PML}_{\forall, \leq}:\langle a\rangle_{0.8}\left(\langle b\rangle_{1} \text { true } \vee\langle d\rangle_{1} \text { true }\right) \\
& \mathrm{PML}_{\forall, \mathrm{I}}: \quad\langle a\rangle_{[0.7,0.8]}\left(\langle b\rangle_{[1,1]} \text { true } \vee\langle d\rangle_{[1,1]} \text { true }\right)
\end{aligned}
$$

where as usual $\phi_{1} \vee \phi_{2}$ stands for $\neg\left(\neg \phi_{1} \wedge \neg \phi_{2}\right)$. The presence of the logical

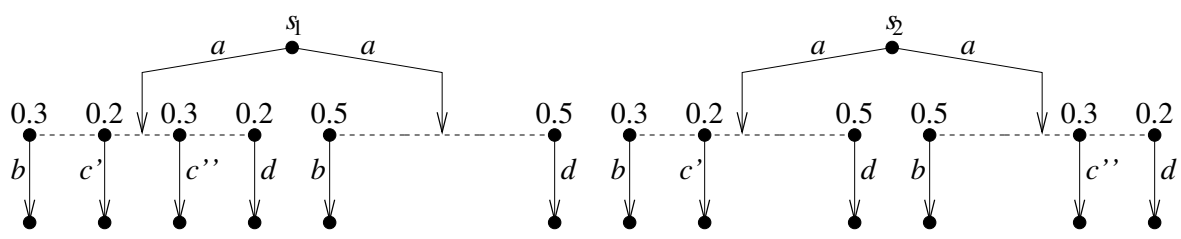

Fig. 4. Models related by $\sim_{\mathrm{PB}, \mathrm{cbc},=}$ and distinguished by all PML variants 
disjunction in the distinguishing formulae above clearly indicates that - having anticipated the quantification over the target states - it is necessary to group equivalence classes together if one wants to obtain the same identifications as the equivalences induced by the variants of PML.

\section{A.2 Group-by-Group Bisimilarities and Extremal Probabilities}

The group-by-group probabilistic bisimilarities of Def. 6 are directly characterized by the existentially interpreted variants of PML. We consider below variants of the group-by-group approach in which only the supremum $(\sqcup)$ and/or the infimum $(\sqcap)$ of the probabilities of reaching a certain group after a certain action are considered. It turns out that the resulting probabilistic bisimilarities are directly characterized by the universally interpreted variants of PML.

Definition 8. Let $(S, A, \longrightarrow)$ be an NPLTS. An equivalence relation $\mathcal{B}$ over $S$ is a பП-group-by-group probabilistic bisimulation iff, whenever $\left(s_{1}, s_{2}\right) \in \mathcal{B}$, then for all actions $a \in A$ and groups of equivalence classes $\mathcal{G} \in 2^{S / \mathcal{B}}$ it holds that $s_{1} \stackrel{a}{\longrightarrow}$ implies $s_{2} \stackrel{a}{\longrightarrow}$ with:

$$
\begin{aligned}
& \bigsqcup_{s_{1}} \mathcal{D}_{1}(\bigcup \mathcal{G})=\mathcal{D}_{1} \bigsqcup_{s_{2}} \mathcal{D}_{a} \mathcal{D}_{2}(\bigcup \mathcal{G}) \\
& \prod_{a} \mathcal{D}_{1}(\bigcup \mathcal{G})=\stackrel{\prod_{a}}{ } \mathcal{D}_{2}(\bigcup \mathcal{G})
\end{aligned}
$$

We denote by $\sim_{\mathrm{PB}, \mathrm{gbg}, \sqcup \sqcap}$ the largest $\sqcup \sqcap$-group-by-group probabilistic bisimulation.

Theorem 8. Let $(S, A, \longrightarrow)$ be an image-finite NPLTS satisfying the minimal probability assumption. Let $s_{1}, s_{2} \in S$. Then:

$$
s_{1} \sim_{\mathrm{PB}, \mathrm{gbg}, \sqcup \sqcap} s_{2} \Longleftrightarrow s_{1} \sim_{\mathrm{PML}_{\forall, \mathrm{I}}} s_{2}
$$

Definition 9. Let $(S, A, \longrightarrow)$ be an NPLTS and symbol $\# \in\{\sqcup, \sqcap\}$. An equivalence relation $\mathcal{B}$ over $S$ is a \#-group-by-group probabilistic bisimulation iff, whenever $\left(s_{1}, s_{2}\right) \in \mathcal{B}$, then for all actions $a \in A$ and groups of equivalence classes $\mathcal{G} \in 2^{S / \mathcal{B}}$ it holds that $s_{1} \stackrel{a}{\longrightarrow}$ implies $s_{2} \stackrel{a}{\longrightarrow}$ with:

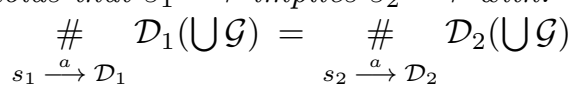

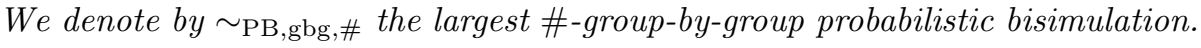

Theorem 9. Let $(S, A, \longrightarrow)$ be an image-finite NPLTS satisfying the minimal probability assumption. Let $s_{1}, s_{2} \in S$. Then:

$$
\begin{aligned}
& s_{1} \sim \mathrm{PB}, \mathrm{gbg}, \sqcup s_{2} \Longleftrightarrow s_{1} \sim_{\mathrm{PML}_{\forall, \leq}} s_{2} \\
& s_{1} \sim \mathrm{PB}, \mathrm{gbg}, \sqcap s_{2} \Longleftrightarrow s_{1} \sim_{\mathrm{PML}_{\forall, \geq}} s_{2}
\end{aligned}
$$

\section{A.3 Relating the Various Probabilistic Bisimilarities}

If we investigate the spectrum of relations considered so far, we discover that five of the six group-by-group probabilistic bisimilarities boil down to the same equivalence, and this extends to the corresponding PML-based equivalences. 
Theorem 10. Let $\mathcal{U}=(S, A, \longrightarrow)$ be an NPLTS and $s_{1}, s_{2} \in S$. Then:

1. $s_{1} \sim_{\mathrm{PB}, \mathrm{dis}} s_{2} \Longrightarrow s_{1} \sim_{\mathrm{PB}, \mathrm{gbg},=s_{2}} \Longrightarrow s_{1} \sim_{\mathrm{PB}, \mathrm{gbg}, \sqcup \sqcap} s_{2}$.

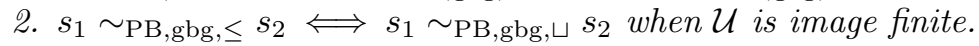

3. $s_{1} \sim \mathrm{PB}, \mathrm{gbg}, \geq s_{2} \Longleftrightarrow s_{1} \sim \mathrm{PB}, \mathrm{gbg}, \sqcap s_{2}$ when $\mathcal{U}$ is image finite.

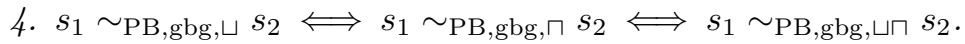

The two implications above cannot be reversed: Fig. 1 shows that $\sim_{\mathrm{PB}, \mathrm{dis}}$ is strictly finer than $\sim_{\mathrm{PB}, \mathrm{gbg},=}$ and Fig. 2 shows that $\sim_{\mathrm{PB}, \mathrm{gbg},=}$ is strictly finer than $\sim_{\mathrm{PB}, \mathrm{gbg}, \sqcup \sqcap .}$. Note that the result relating $\sim_{\mathrm{PB}, \mathrm{gbg}, \sqcup,}, \sim_{\mathrm{PB}, \mathrm{gbg}, \sqcap}$, and $\sim_{\mathrm{PB}, \mathrm{gbg}, \sqcup \sqcap}$ holds because groups of equivalence classes are considered. Analogous bisimilarities defined in a class-by-class fashion would not coincide.

Another interesting property is that the five coinciding group-by-group probabilistic bisimilarities are the same as their ct-variants, and hence are insensitive to whether deterministic or randomized schedulers are employed to resolve nondeterminism. This is not the case with $\sim_{\mathrm{PB}, \mathrm{dis}}$ and $\sim_{\mathrm{PB}, \mathrm{gbg},=}$. Moreover, the ct-variants of all the six group-by-group probabilistic bisimilarities boil down to the same equivalence $\left(\sim_{\mathrm{PB}, \mathrm{gbg}, \leq}\right)$, meaning that, in the bisimulation game, randomized schedulers reduce the discriminating power of the $=$-comparison of probabilities to that of the $\leq$-comparison.

Theorem 11. Let $\mathcal{U}=(S, A, \longrightarrow)$ be an NPLTS and $s_{1}, s_{2} \in S$. Then:

1. $s_{1} \sim \mathrm{PB}, \triangleleft s_{2} \Longrightarrow s_{1} \sim_{\mathrm{PB}, \triangleleft}^{\mathrm{ct}} s_{2}$ for $\triangleleft \in\{"$ dis", "gbg, $="\}$.

2. $s_{1} \sim_{\mathrm{PB}, \mathrm{gbg}, \triangleright} s_{2} \Longleftrightarrow s_{1} \sim_{\mathrm{PB}, \mathrm{gbg}, \triangleright}^{\mathrm{ct}} s_{2}$ for $\triangleright \in\{\leq, \geq, \sqcup \sqcap, \sqcup, \sqcap\}$ when $\mathcal{U}$ is image finite.

3. $s_{1} \sim_{\mathrm{PB}, \mathrm{dis}}^{\mathrm{ct}} s_{2} \Longrightarrow s_{1} \sim_{\mathrm{PB}, \mathrm{gbg},=}^{\mathrm{ct}}=s_{2}$.

4. $s_{1} \sim_{\mathrm{PB}, \mathrm{gbg},=}^{\mathrm{ct}}=s_{2} \Longleftrightarrow s_{1} \sim_{\mathrm{PB}, \mathrm{gbg}, \sqcup \sqcap}^{\mathrm{ct}} s_{2}$ when $\mathcal{U}$ is image finite.

The inclusions of $\sim_{\mathrm{PB}, \mathrm{dis}}$ and $\sim_{\mathrm{PB}, \mathrm{gbg},=}$ in $\sim_{\mathrm{PB}, \mathrm{dis}}^{\mathrm{ct}}$ and $\sim_{\mathrm{PB}, \mathrm{gbg},=}^{\mathrm{ct}}$, respectively, are strict, as shown by Fig. 2; the central offer-transition of $s_{1}$ can be matched by a convex combination of the two offer-transitions of $s_{2}$ both weighted by 0.5. Moreover, Fig. 1 shows that the inclusion of $\sim_{\mathrm{PB}, \mathrm{dis}}^{\mathrm{ct}}$ in $\sim_{\mathrm{PB}, \mathrm{gbg}}^{\mathrm{ct}}=$ is strict. Finally, Figs. 1 and 2 show that $\sim_{\mathrm{PB}, \mathrm{dis}}^{\text {ct }}$ and $\sim_{\mathrm{PB}, \mathrm{gbg},=}$ are incomparable with each other.

\section{A.4 Multistep Variants of Probabilistic Bisimilarities}

Further relations can be defined by considering entire computations instead of individual transitions in the bisimulation game. Given an $\operatorname{NPLTS} \mathcal{U}=(S, A, \longrightarrow)$, we say that $c \equiv s_{0} \stackrel{a_{1}}{\longrightarrow} s_{1} \stackrel{a_{2}}{\longrightarrow} s_{2} \ldots s_{n-1} \stackrel{a_{n}}{\longrightarrow} s_{n}$ is a computation of $\mathcal{U}$ of length $n$ going from $s_{0}$ to $s_{n}$ iff for all $i=1, \ldots, n$ there exists a transition $s_{i-1} \stackrel{a_{i}}{\longrightarrow} \mathcal{D}_{i}$ such that $s_{i} \in \operatorname{supp}\left(\mathcal{D}_{i}\right)$, with $\mathcal{D}_{i}\left(s_{i}\right)$ being the execution probability of step $s_{i-1} \stackrel{a_{i}}{\longrightarrow} s_{i}$ of $c$ conditioned on the selection of transition $s_{i-1} \stackrel{a_{i}}{\longrightarrow} \mathcal{D}_{i}$ of $\mathcal{U}$ at state $s_{i-1}$. We call combined computation a computation in which every step arises from a combined transition. 
The multistep variants of probabilistic bisimilarities for NPLTS models can be defined in different ways. The first option, inspired by bisimilarity for fully nondeterministic processes, consists of changing the one-step definitions by considering traces $\alpha \in A^{*}$ in place of actions $a \in A$ and $\stackrel{\alpha}{\Longrightarrow}$ in place of $\stackrel{a}{\longrightarrow}$ (resp. $\stackrel{\alpha}{\Longrightarrow}$ c in place of $\stackrel{a}{\longrightarrow}$ ), where $s \stackrel{\alpha}{\Longrightarrow} \mathcal{D}$ means that there exists a computation from $s$ labeled with $\alpha$ whose last step is originated by a transition reaching distribution $\mathcal{D}$. When $\alpha$ is the empty sequence $\varepsilon$, we let $s \stackrel{\varepsilon}{\Longrightarrow} \delta_{s}$. It was shown in [12] that the discriminating power of bisimilarity for fully nondeterministic processes does not change if the multistep transition relation $\stackrel{\alpha}{\Longrightarrow}$ is used instead of the one-step relation $\stackrel{a}{\longrightarrow}$. As expected, this result carries over class-distribution and group-by-group probabilistic bisimilarities for nondeterministic and probabilistic processes (see App. C).

The second option, inspired by probabilistic bisimilarity for reactive probabilistic processes, does not only compare the probability values arising from the last step of the computations, but additionally considers the probability of performing the entire computations. While it can be shown that the discriminating power of the probabilistic bisimilarity for reactive probabilistic processes in [16] and of class-distribution probabilistic bisimilarities for nondeterministic and probabilistic processes does not change if multistep probability values are compared instead of one-step values, this is not the case with the group-by-group probabilistic bisimilarities (see App. D).
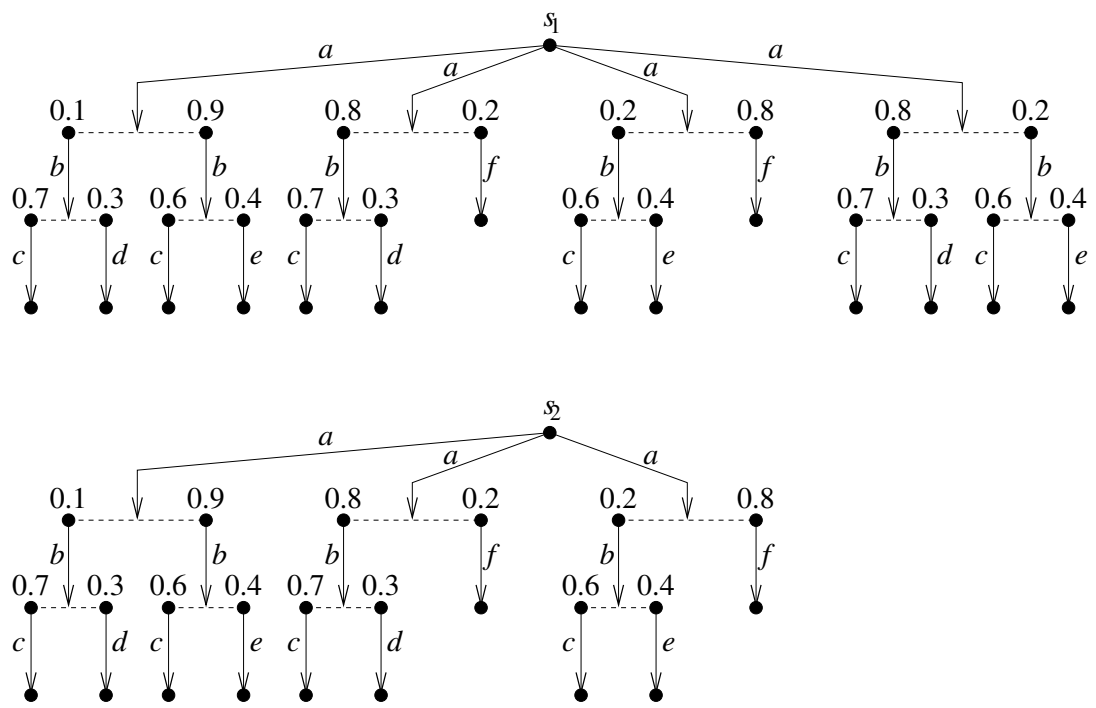

Fig. 5. Two models identified by $\sim_{\mathrm{PB}, \mathrm{gbg},=}$ and $\sim_{\mathrm{PB}, \mathrm{gbg}, \leq}$ that are distinguished by PCTL* 
Finally, the third option, which is orthogonal to the previous two, consists of imposing some constraints along the computations, such as passing through specific sets of states at each step. This is the idea exploited in [25] in order to define probabilistic bisimilarities - following the second option above - over nondeterministic and probabilistic Kripke structures that are precisely characterized by PCTL, PCTL*, and their variants without the next-time operator, as interpreted in [3]. We note that the strong 1-depth bisimulation in [25] and our $\sim_{\mathrm{PB}, \mathrm{gbg}, \leq} \leq$ are strongly related. In contrast, the probabilistic bisimilarities built on the strong 1-depth bisimulation in [25] are finer than our group-bygroup probabilistic bisimilarities. Consider for instance the two NPLTS models in Fig. 5 . We have that $s_{1} \sim_{\mathrm{PB}, \mathrm{gbg},=} s_{2}-$ and hence $s_{1} \sim_{\mathrm{PB}, \mathrm{gbg}, \leq} s_{2}-$ as witnessed by the equivalence relation that pairs states with identically labeled transitions and, in the case of $b$-transitions, identical target distributions. However, $s_{1}$ and $s_{2}$ are distinguished by the probabilistic bisimilarity in [25] that is characterized by PCTL*. In fact, let us view the two NPLTS models as two nondeterministic and probabilistic Kripke structures by eliminating actions from transitions and labeling each state with the set of its next-actions. Then the PCTL* formula $\operatorname{Pr}_{<0.61}(\mathbf{X X} c)$ is satisfied by $s_{2}$ but it is not satisfied by $s_{1}$, because the probability of reaching in two steps a state that enables $c$ in the maximal resolution of $s_{1}$ starting with the rightmost $a$-transition is $0.8 \cdot 0.7+0.2 \cdot 0.6=0.68$ and hence it is greater than 0.61 . 


\section{B Proofs of Results}

Proof of Thm. 1. Directly from Thm. 10.

Proof of Thm. 2. Directly from Thm. 11.

Proof of Thm. 3. Since every transition of this specific NPLTS can reach with probability greater than 0 a single state and hence a single class of any equivalence relation - which are thus reached with probability 1 - the reflexive, symmetric, and transitive closure of a bisimulation is trivially a =-groupby-group (ct-)probabilistic bisimulation, a $\leq$-group-by-group (ct-)probabilistic bisimulation, and a $\geq$-group-by-group (ct-)probabilistic bisimulation.

Proof of Thm. 4. Since every state of this specific NPLTS has at most one transition labeled with a certain action, a probabilistic bisimulation is trivially a =-group-by-group (ct-)probabilistic bisimulation, a $\leq$-group-by-group (ct-)probabilistic bisimulation, and a $\geq$-group-by-group (ct-)probabilistic bisimulation.

Proof of Thm. 5. Since every state of this specific NPLTS has either zero or more Dirac transitions or a single non-Dirac transition, a class-distribution (ct-)probabilistic bisimulation is trivially a =-group-by-group (ct-)probabilistic bisimulation, a s-group-by-group (ct-)probabilistic bisimulation, and a $\geq$-group-by-group (ct-)probabilistic bisimulation.

Proof of Lemma 1. Denoting by $\sim^{\prime}$ the relation $\bigcap_{i \in \mathbb{N}} \sim_{\mathrm{PB}, \mathrm{gbg},=}^{i}$, we prove

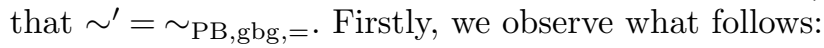

$-\sim^{\prime}$ is an equivalence relation because so is $\sim_{\mathrm{PB}, \mathrm{gbg},=}^{i}$ for all $i \in \mathbb{N}$.

- Given $C \in S / \sim^{\prime}$ and $i \in \mathbb{N}$, there exists a unique element $C_{i}$ in $S / \sim_{\mathrm{PB}, \mathrm{gbg},=}^{i}$ such that $C_{i} \supseteq C$, and hence $C=\bigcap_{i \in \mathbb{N}} C_{i}$ with $C_{i_{1}} \supseteq C_{i_{2}}$ for $i_{1} \leq i_{2}$.

- As a consequence, given $\mathcal{G} \in 2^{S / \sim^{\prime}}$ and $i \in \mathbb{N}$, there exists a unique element $\mathcal{G}_{i}$ in $2^{S / \sim_{\mathrm{PB}, \mathrm{gbg},=}^{i}}$ such that every class in $\mathcal{G}_{i}$ contains some class in $\mathcal{G}$, and hence $\bigcup \mathcal{G}=\bigcap_{i \in \mathbb{N}}\left(\bigcup \mathcal{G}_{i}\right)$ with $\bigcup \mathcal{G}_{i_{1}} \supseteq \bigcup \mathcal{G}_{i_{2}}$ for $i_{1} \leq i_{2}$.

- Moreover, if $s \stackrel{a}{\longrightarrow} \mathcal{D}$, then $\mathcal{D}(\bigcup \mathcal{G})=\inf _{i \in \mathbb{N}} \mathcal{D}\left(\cup \mathcal{G}_{i}\right)$. In fact, observing that for all $i \in \mathbb{N}$ it holds that $\mathcal{D}\left(\bigcup \mathcal{G}_{i}\right) \geq \mathcal{D}(\bigcup \mathcal{G})$ because $\bigcup \mathcal{G}_{i} \supseteq \bigcup \mathcal{G}$, if we let $p=\inf _{i \in \mathbb{N}} \mathcal{D}\left(\bigcup \mathcal{G}_{i}\right)$, then $p \geq \mathcal{D}(\bigcup \mathcal{G})$ because $\mathcal{D}(\bigcup \mathcal{G})$ is a lower bound of the sequence $\left(\mathcal{D}\left(\bigcup \mathcal{G}_{i}\right)\right)_{i \in \mathbb{N}}$ and $p$ is the greatest lower bound of that sequence. Suppose $p>\mathcal{D}(\bigcup \mathcal{G})$ and let $\delta=p-\mathcal{D}(\bigcup \mathcal{G})$. Since $\delta>0$ and the summation $\mathcal{D}(S \backslash \cup \mathcal{G})$ satisfies $\sum_{s \in S \backslash \cup \mathcal{G}} \mathcal{D}(s) \leq 1$ and hence converges, there exists a finite subset $X$ of $S \backslash \bigcup \mathcal{G}$ such that the rest $\mathcal{D}((S \backslash \bigcup \mathcal{G}) \backslash X)$ of the previously considered summation satisfies $\sum_{s \in(S \backslash \cup \mathcal{G}) \backslash X} \mathcal{D}(s)<\delta$. Let $Y=(S \backslash \bigcup \mathcal{G}) \backslash X$. For all $i \in \mathbb{N}$, it holds that:

$$
\cup \mathcal{G}_{i}=\bigcup \mathcal{G} \cup\left(Y \cap \bigcup \mathcal{G}_{i}\right) \cup\left(X \cap \bigcup \mathcal{G}_{i}\right)
$$

where the three sets on the right-hand side are pairwise disjoint and hence:

$$
\begin{aligned}
\mathcal{D}\left(\bigcup \mathcal{G}_{i}\right) & =\mathcal{D}(\bigcup \mathcal{G})+\mathcal{D}\left(Y \cap \bigcup \mathcal{G}_{i}\right)+\mathcal{D}\left(X \cap \bigcup \mathcal{G}_{i}\right) \\
& \leq \mathcal{D}(\bigcup \mathcal{G})+\mathcal{D}(Y)+\mathcal{D}\left(X \cap \bigcup \mathcal{G}_{i}\right) \\
& <\mathcal{D}(\bigcup \mathcal{G})+\delta+\mathcal{D}\left(X \cap \bigcup \mathcal{G}_{i}\right) \\
& =p+\mathcal{D}\left(X \cap \bigcup \mathcal{G}_{i}\right)
\end{aligned}
$$


From the inequality above and $\mathcal{D}\left(\bigcup \mathcal{G}_{i}\right) \geq p$, we derive that:

$$
\mathcal{D}\left(X \cap \bigcup \mathcal{G}_{i}\right)>\mathcal{D}\left(\bigcup \mathcal{G}_{i}\right)-p \geq 0
$$

and hence $X \cap \bigcup \mathcal{G}_{i} \neq \emptyset$ for all $i \in \mathbb{N}$. As a consequence, $X \cap \bigcup \mathcal{G} \neq \emptyset$ because $X$ is finite and $\bigcup \mathcal{G}_{0} \supseteq \bigcup \mathcal{G}_{1} \supseteq \ldots$. This contradicts the fact that $X$ is a subset of $S \backslash \bigcup \mathcal{G}$. Therefore, it must be $p=\mathcal{D}(\bigcup \mathcal{G})$.

Secondly, it holds that $\sim^{\prime} \supseteq \sim_{\mathrm{PB}, \mathrm{gbg},=}$ because $\sim_{\mathrm{PB}, \mathrm{gbg},=}^{i} \supseteq \sim_{\mathrm{PB}, \mathrm{gbg},=}$ for all $i \in \mathbb{N}$ as we now show by proceeding by induction on $i$ :

- If $i=0$, then $\sim_{\mathrm{PB}, \mathrm{gbg},=}^{i}=S \times S \supseteq \sim_{\mathrm{PB}, \mathrm{gbg},=}$.

- Let $i$ be an element of $\mathbb{N}$ for which the result holds and consider $i+1$. If $s_{1}, s_{2} \in S$ satisfy $s_{1} \sim_{\mathrm{PB}, \mathrm{gbg},=} s_{2}$, then:

- For all $a \in A$ and $\mathcal{G} \in 2^{S / \sim_{\mathrm{PB}, \mathrm{gbg},=}}$, it holds that for each $s_{1} \stackrel{a}{\longrightarrow} \mathcal{D}_{1}$ there exists $s_{2} \stackrel{a}{\longrightarrow} \mathcal{D}_{2}$ such that $\mathcal{D}_{1}(\bigcup \mathcal{G})=\mathcal{D}_{2}(\bigcup \mathcal{G})$.

- $s_{1} \sim_{\mathrm{PB}, \mathrm{gbg},=}^{i} s_{2}$ because $\sim_{\mathrm{PB}, \mathrm{gbg},=}^{i}=\supseteq \sim_{\mathrm{PB}, \mathrm{gbg},=}$ by the induction hypothesis.

Since every equivalence class of $\sim_{\mathrm{PB}, \mathrm{gbg},=}^{i}$ is equal to the union of some equivalence classes of $\sim_{\mathrm{PB}, \mathrm{gbg},=}$ and hence the union of equivalence classes in every group $\mathcal{G}^{\prime}$ of $\sim_{\mathrm{PB}, \mathrm{gbg},=}^{i}$ is equal to the union of the equivalence classes in some group $\mathcal{G}$ of $\sim_{\mathrm{PB}, \mathrm{gbg},=}$, we derive that for all $a \in A$ and $\mathcal{G}^{\prime} \in 2^{S / \sim_{\mathrm{PB}, \mathrm{gbg},=}^{i}}$ it holds that for each $s_{1} \stackrel{a}{\longrightarrow} \mathcal{D}_{1}$ there exists $s_{2} \stackrel{a}{\longrightarrow} \mathcal{D}_{2}$ such that:

This means that $s_{1} \sim_{\mathrm{PB}, \mathrm{gbg},=}^{i+1} s_{2}$.

$$
\mathcal{D}_{1}\left(\bigcup \mathcal{G}^{\prime}\right)=\mathcal{D}_{1}(\bigcup \mathcal{G})=\mathcal{D}_{2}(\bigcup \mathcal{G})=\mathcal{D}_{2}\left(\bigcup \mathcal{G}^{\prime}\right)
$$

Thirdly, we prove that $\sim^{\prime} \subseteq \sim_{\mathrm{PB}, \text { gbg, }}$, by showing that $\sim^{\prime}$ is a =-group-by-group probabilistic bisimulation. Suppose that $s_{1}, s_{2} \in S$ satisfy $s_{1} \sim^{\prime} s_{2}$ and, given $a \in A$ and $\mathcal{G} \in 2^{S / \sim^{\prime}}$, assume that $s_{1} \stackrel{a}{\longrightarrow} \mathcal{D}_{1}$. Then $\mathcal{D}_{1}(\bigcup \mathcal{G})=\inf _{i \in \mathbb{N}} \mathcal{D}_{1}\left(\bigcup \mathcal{G}_{i}\right)$ where each $\mathcal{G}_{i}$ is the unique element in $2^{S / \sim_{\mathrm{PB}, \mathrm{gbg},}^{i}=}$ such that every class in $\mathcal{G}_{i}$ contains some class in $\mathcal{G}$.

Observing that $\sim_{\mathrm{PB}, \mathrm{gbg},=}^{0}$ induces a single equivalence class equal to $S$ and hence $\mathcal{D}_{1}\left(\cup \mathcal{G}_{0}\right)=\mathcal{D}_{1}(S)=1$, from $s_{1} \sim^{\prime} s_{2}$ and $s_{1} \stackrel{a}{\longrightarrow} \mathcal{D}_{1}$ it follows that for all $i \in \mathbb{N}_{\geq 1}$ there exists $s_{2} \stackrel{a}{\longrightarrow} \mathcal{D}_{2, i}$ such that $\mathcal{D}_{1}\left(\cup \mathcal{G}_{i-1}\right)=\mathcal{D}_{2, i}\left(\cup \mathcal{G}_{i-1}\right)$. Since the NPLTS is image finite, the set $\left\{\mathcal{D}_{2, i} \mid i \in \mathbb{N}_{\geq 1}\right\}$ is finite and we enumerate it as $\left\{D_{2}^{1}, \ldots, D_{2}^{k}\right\}$. For each $j \in\{1, \ldots, k\}$, we also let $I_{j}$ be the set of indexes $i \in \mathbb{N}_{\geq 1}$ such that $D_{1}\left(\bigcup \mathcal{G}_{i-1}\right)=D_{2}^{j}\left(\bigcup \mathcal{G}_{i-1}\right)$. At least one set in $\left\{I_{1}, \ldots, I_{k}\right\}$ is infinite. Indeed, if every $I_{j}$ were finite, then there would exist an integer $i$ such that $i \notin I_{j}$ for each $j \in\{1, \ldots, k\}$. Hence, there would exists a group $\mathcal{G}_{i-1}$ such that $D_{1}\left(\bigcup \mathcal{G}_{i-1}\right) \neq D_{2}^{j}\left(\bigcup \mathcal{G}_{i-1}\right)$ for each $j \in\{1, \ldots, k\}$. However, this implies $s_{1} \chi_{\mathrm{PB}, \mathrm{gbg},=}^{i} s_{2}$, which whould contradict the assumption $s_{1} \sim^{\prime} s_{2}$.

Let $j^{\prime}$ be such that $I_{j^{\prime}}$ is infinite. We have that:

$$
\begin{aligned}
D_{1}(\mathcal{G}) & =\inf _{i \in \mathbb{N}} \mathcal{D}_{1}\left(\bigcup \mathcal{G}_{i}\right) \\
& =\inf _{i \in I_{j^{\prime}}} \mathcal{D}_{1}\left(\bigcup \mathcal{G}_{i}\right) \\
& =\inf _{i \in I_{j^{\prime}}} \mathcal{D}_{2}^{j^{\prime}}\left(\bigcup \mathcal{G}_{i}\right) \\
& =\inf _{i \in \mathbb{N}} \mathcal{D}_{2}^{j^{\prime}}\left(\bigcup \mathcal{G}_{i}\right)=D_{2}^{j^{\prime}}(\mathcal{G})
\end{aligned}
$$


where the equalities (1) and (2) above derive from the fact that $\left(D_{1}\left(\mathcal{G}_{i}\right)\right)_{i \in I_{j}^{\prime}}$ and $\left(D_{2}^{j^{\prime}}\left(\mathcal{G}_{i}\right)\right)_{i \in I_{i}^{\prime}}$ are infinite subsequences of $\left(D_{1}\left(\mathcal{G}_{i}\right)\right)_{i \in \mathbb{N}}$ and $\left(D_{2}^{j^{\prime}}\left(\mathcal{G}_{i}\right)\right)_{i \in \mathbb{N}}$, respectively, and therefore they have the same infimum.

In conclusion, we have that $\sim^{\prime}$ is a =-group-by-group probabilistic bisimulation.

Proof of Lemma 2. Given an image-finite NPLTS $(S, A, \longrightarrow)$ satisfying the minimal probability assumption, and given $s_{1}, s_{2} \in S$, we proceed by induction on $i \in \mathbb{N}$.

Base of Induction $(i=0)$ : Since $\sim_{\mathrm{PB}, \mathrm{gbg},=}^{0}=S \times S$ and $\mathcal{F}_{\mathrm{PML} \exists, \mathrm{I}}^{0}(s)=\{\phi \in$ $\mathbb{F}_{\mathrm{PML}_{\exists}, \mathrm{I}}^{0} \mid \phi \equiv$ true $\}$ for all $s \in S$, it trivially holds that:

$$
s_{1} \sim_{\mathrm{PB}, \mathrm{gbg},=}^{0} s_{2} \Longleftrightarrow \mathcal{F}_{\mathrm{PML}_{\exists, \mathrm{I}}}^{0}\left(s_{1}\right)=\mathcal{F}_{\mathrm{PML} \exists, \mathrm{I}}^{0}\left(s_{2}\right)
$$

Induction Hypothesis: Given $i \in \mathbb{N}$, we assume that for all $j=0, \ldots, i$ :

$$
s_{1} \sim_{\mathrm{PB}, \mathrm{gbg},=}^{j} s_{2} \Longleftrightarrow \mathcal{F}_{\mathrm{PML}}^{j}\left(s_{1}\right)=\mathcal{F}_{\mathrm{PML}}^{j}\left(s_{2}\right)
$$

Induction Step: We prove both implications for $i+1$ by reasoning on their corresponding contrapositive statements, i.e., we prove that:

$$
\mathcal{F}_{\mathrm{PML}}^{i+1, \mathrm{I}}\left(s_{1}\right) \neq \mathcal{F}_{\mathrm{PML}}^{i+1, \mathrm{I}}\left(s_{2}\right) \Longleftrightarrow s_{1} \mathcal{\chi}_{\mathrm{PB}, \mathrm{gbg},=}^{i+1} s_{2}
$$

$(\Longrightarrow)$ If $\mathcal{F}_{\mathrm{PML}_{\exists, \mathrm{I}}}^{i+1}\left(s_{1}\right) \neq \mathcal{F}_{\mathrm{PML}_{\exists, \mathrm{I}}}^{i+1}\left(s_{2}\right)$, then there are two cases:

- If $\mathcal{F}_{\mathrm{PML}_{\exists, \mathrm{I}}}^{i}\left(s_{1}\right) \neq \mathcal{F}_{\mathrm{PML}_{\exists, \mathrm{I}}}^{i}\left(s_{2}\right)$, then by the induction hypothesis it holds that $s_{1} \chi_{\mathrm{PB}, \mathrm{gbg},=}^{i} s_{2}$ and hence $s_{1} \chi_{\mathrm{PB}, \mathrm{gbg},=}^{i+1} s_{2}$.

- If $\mathcal{F}_{\mathrm{PML}_{\exists, \mathrm{I}}}^{i}\left(s_{1}\right)=\mathcal{F}_{\mathrm{PML}_{\exists, \mathrm{I}}}^{i}\left(s_{2}\right)$, then from $\mathcal{F}_{\mathrm{PML}_{\exists, \mathrm{I}}}^{i+1}\left(s_{1}\right) \neq \mathcal{F}_{\mathrm{PML}_{\exists, \mathrm{I}}}^{i+1}\left(s_{2}\right)$ it follows that there exists $\phi \in \mathbb{F}_{\mathrm{PML}_{\exists, \mathrm{I}}}^{i+1}$ such that $s_{1} \in \mathcal{M}_{\mathrm{PML}_{\exists, \mathrm{I}}} \llbracket \phi \rrbracket$ and $s_{2} \notin$ $\mathcal{M}_{\mathrm{PML}_{\exists, \mathrm{I}}} \llbracket \phi \rrbracket$. We now proceed by induction on the syntactical structure of $\phi$. Here we only consider the case $\phi=\langle a\rangle_{\left[p_{1}, p_{2}\right]} \phi^{\prime}$ because the other cases are routine.

From $s_{1} \in \mathcal{M}_{\mathrm{PML}_{\exists, \mathrm{I}}} \llbracket\langle a\rangle_{\left[p_{1}, p_{2}\right]} \phi^{\prime} \rrbracket$ and $s_{2} \notin \mathcal{M}_{\mathrm{PML}_{\exists, \mathrm{I}}} \llbracket\langle a\rangle_{\left[p_{1}, p_{2}\right]} \phi^{\prime} \rrbracket$, it follows that:

- $p_{1} \leq \mathcal{D}_{1}\left(\mathcal{M}_{\mathrm{PML}_{\exists . \mathrm{I}}} \llbracket \phi^{\prime} \rrbracket\right) \leq p_{2}$ for some $\mathcal{D}_{1}$ such that $s_{1} \stackrel{a}{\longrightarrow} \mathcal{D}_{1}$.

- $\mathcal{D}_{2}\left(\mathcal{M}_{\mathrm{PML}_{\exists, \mathrm{I}}} \llbracket \phi^{\prime} \rrbracket\right)<p_{1}$ or $\mathcal{D}_{2}\left(\mathcal{M}_{\mathrm{PML}_{\exists, \mathrm{I}}} \llbracket \phi^{\prime} \rrbracket\right)>p_{2}$ for all $\mathcal{D}_{2}$ such that $s_{2} \stackrel{a}{\longrightarrow} \mathcal{D}_{2}$.

Since $\phi^{\prime} \in \mathbb{F}_{\mathrm{PML} \exists, \mathrm{I}}^{i}$, by the induction hypothesis there exists $\mathcal{G} \in 2^{S / \sim_{\mathrm{PB}, \mathrm{gbg},=}^{i}}$ such that $\bigcup_{C \in \mathcal{G}} C=\mathcal{M}_{\mathrm{PML}_{\exists, \mathrm{I}}} \llbracket \phi^{\prime} \rrbracket$. Then:

- $\mathcal{D}_{1}(\bigcup \mathcal{G})=q \in \mathbb{R}_{\left[p_{1}, p_{2}\right]}$.

- $\mathcal{D}_{2}(\bigcup \mathcal{G}) \neq q$ for all $\mathcal{D}_{2}$ such that $s_{2} \stackrel{a}{\longrightarrow} \mathcal{D}_{2}$.

Therefore $s_{1} \chi_{\mathrm{PB}, \mathrm{gbg},=}^{i+1} s_{2}$.

$(\Longleftarrow)$ If $s_{1} \chi_{\mathrm{PB}, \mathrm{gbg},=}^{i+1} s_{2}$, then there are two cases:

- If $s_{1} \chi_{\mathrm{PB}, \mathrm{gbg},=}^{i} s_{2}$, then by the induction hypothesis it holds that $\mathcal{F}_{\mathrm{PML} \exists, \mathrm{I}}^{i}\left(s_{1}\right)$ $\neq \mathcal{F}_{\mathrm{PML}_{\exists, \mathrm{I}}}^{i}\left(s_{2}\right)$ and hence $\mathcal{F}_{\mathrm{PML}_{\exists, \mathrm{I}}}^{i+1}\left(s_{1}\right) \neq \mathcal{F}_{\mathrm{PML}_{\exists, \mathrm{I}}}^{i+1}\left(s_{2}\right)$.

- If $s_{1} \sim_{\mathrm{PB}, \mathrm{gbg},=}^{i} s_{2}$, then from $s_{1} \mathcal{\chi}_{\mathrm{PB}, \mathrm{gbg},=}^{i+1} s_{2}$ it follows that there exist $p \in \mathbb{R}_{[0,1]}$ and $\mathcal{G} \in 2^{S / \sim_{\mathrm{PB}, \mathrm{gbg},=}^{i}}$ such that: 
- $\mathcal{D}_{1}(\bigcup \mathcal{G})=p$ for some $\mathcal{D}_{1}$ such that $s_{1} \stackrel{a}{\longrightarrow} \mathcal{D}_{1}$.

- $\mathcal{D}_{2}(\bigcup \mathcal{G}) \neq p$ for all $\mathcal{D}_{2}$ such that $s_{2} \stackrel{a}{\longrightarrow} \mathcal{D}_{2}$.

Let $\mathcal{G}_{1}=\left\{C \in S / \sim_{\mathrm{PB}, \mathrm{gbg},=}^{i} \mid \mathcal{D}_{1}(C)>0\right\}$ and $\mathcal{G}_{2}=\left\{C \in S / \sim_{\mathrm{PB}, \mathrm{gbg},=}^{i} \mid\right.$ $\left.\exists \mathcal{D}_{2} . s_{2} \stackrel{a}{\longrightarrow} \mathcal{D}_{2} \wedge \mathcal{D}_{2}(C)>0\right\}$. Thanks to the assumptions of image finiteness and minimal probability, both $\mathcal{G}_{1}$ and $\mathcal{G}_{2}$ are finite.

By the induction hypothesis, there exists a distinguishing formula $\phi_{\left\langle C_{1}, C_{2}\right\rangle} \in$ $\mathbb{F}_{\mathrm{PML} \exists, \mathrm{I}}^{i}$ for all $C_{1}$ and $C_{2}$ in $S / \sim_{\mathrm{PB}, \mathrm{gbg},=}^{i}$ such that $C_{1} \neq C_{2}$, i.e.:

$$
\begin{aligned}
& C_{1} \subseteq \mathcal{M}_{\mathrm{PML}_{\exists, \mathrm{I}}} \llbracket \phi_{<C_{1}, C_{2}>} \rrbracket \\
& C_{2} \cap \mathcal{M}_{\mathrm{PML} \exists, \mathrm{I}} \llbracket \phi_{<C_{1}, C_{2}>} \rrbracket=\emptyset
\end{aligned}
$$

Then:

$$
\phi_{\mathcal{G}}=\bigvee_{C \in \mathcal{G}}\left(\bigwedge_{C_{1} \in \mathcal{G}_{1} \backslash\{C\}} \phi_{<C, C_{1}>} \wedge \bigwedge_{C_{2} \in \mathcal{G}_{2} \backslash\{C\}} \phi_{<C, C_{2}>}\right)
$$

where $\bigvee_{i \in I} \phi_{i}=\neg \bigwedge_{i \in I} \neg \phi_{i}$ for $I$ finite and $\bigwedge_{i \in I} \phi_{i}=$ true for $I=\emptyset$, yields a distinguishing formula for $s_{1}$ and $s_{2}$ because:

- $s_{1} \in \mathcal{M}_{\mathrm{PML}_{\exists, \mathrm{I}}} \llbracket\langle a\rangle_{[p, p]} \phi_{\mathcal{G}} \rrbracket$.

- $s_{2} \notin \mathcal{M}_{\mathrm{PML}_{\exists, \mathrm{I}}} \llbracket\langle a\rangle_{[p, p]} \phi_{\mathcal{G}} \rrbracket$.

Since $\langle a\rangle_{[p, p]} \phi_{\mathcal{G}} \in \mathbb{F}_{\mathrm{PML} \exists, \mathrm{I}}^{i+1}$, we derive that $\mathcal{F}_{\mathrm{PML}_{\exists, \mathrm{I}}}^{i+1}\left(s_{1}\right) \neq \mathcal{F}_{\mathrm{PML} \exists, \mathrm{I}}^{i+1}\left(s_{2}\right)$.

Proof of Thm. 7. The proof of the first result is similar to the proof of Thm. 6 based on Lemmata 1 and 2 - up to the use of $\leq$ in place of $=$ when comparing the probabilities of reaching a group of equivalence classes and the use of $\sim_{\mathrm{PB}, \mathrm{gbg}, \leq}^{i}$, $\mathcal{F}_{\mathrm{PML}_{\exists, \geq}}^{i}, \mathcal{M}_{\mathrm{PML}_{\exists, \geq}}$, and $\langle a\rangle_{p}$ in place of $\sim_{\mathrm{PB}, \mathrm{gbg},=}^{i}, \mathcal{F}_{\mathrm{PML} \exists, \mathrm{I}}^{i}, \mathcal{M}_{\mathrm{PML}_{\exists, \mathrm{I}}}$, and $\langle a\rangle_{\left[p_{1}, p_{2}\right]}$ wherever necessary.

In particular, for the induction step of Lemma 2 we point out that:

- In the $(\Longrightarrow)$ part, from $s_{1} \in \mathcal{M}_{\mathrm{PML}_{\exists, \geq}} \llbracket\langle a\rangle_{p} \phi^{\prime} \rrbracket$ and $s_{2} \notin \mathcal{M}_{\mathrm{PML}_{\exists, \geq}} \llbracket\langle a\rangle_{p} \phi^{\prime} \rrbracket$, it follows that:

- $\mathcal{D}_{1}\left(\mathcal{M}_{\mathrm{PML}_{\exists, \geq}} \llbracket \phi^{\prime} \rrbracket\right) \geq p$ for some $\mathcal{D}_{1}$ such that $s_{1} \stackrel{a}{\longrightarrow} \mathcal{D}_{1}$.

- $\mathcal{D}_{2}\left(\mathcal{M}_{\mathrm{PML}_{\exists, \geq}} \llbracket \phi^{\prime} \rrbracket\right)<p$ for all $\mathcal{D}_{2}$ such that $s_{2} \stackrel{a}{\longrightarrow} \mathcal{D}_{2}$.

Since $\phi^{\prime} \in \mathbb{F}_{\mathrm{PML}_{\exists,},>}^{i}$, by the induction hypothesis there exists $\mathcal{G} \in 2^{S / \sim_{\mathrm{PB}, \mathrm{gbg}}^{i}, \leq}$ such that $\bigcup_{C \in \mathcal{G}} C=\mathcal{M}_{\mathrm{PML}_{\exists, \geq}} \llbracket \phi^{\prime} \rrbracket$. Then:

- $\mathcal{D}_{1}(\bigcup \mathcal{G}) \geq p$.

- $\mathcal{D}_{2}(\bigcup \mathcal{G})<p$ for all $\mathcal{D}_{2}$ such that $s_{2} \stackrel{a}{\longrightarrow} \mathcal{D}_{2}$.

- In the $(\Longleftarrow)$ part, if $s_{1} \sim_{\mathrm{PB}, \mathrm{gbg}, \leq}^{i} s_{2}$, then there exist $p \in \mathbb{R}_{[0,1]}$ and $\mathcal{G} \in$ $2^{S / \sim_{\mathrm{PB}, \mathrm{gbg},}^{i} \leq \mathrm{such} \text { that: }}$

- $\mathcal{D}_{1}(\bigcup \mathcal{G})=p$ for some $\mathcal{D}_{1}$ such that $s_{1} \stackrel{a}{\longrightarrow} \mathcal{D}_{1}$.

- $\mathcal{D}_{2}(\bigcup \mathcal{G})<p$ for all $\mathcal{D}_{2}$ such that $s_{2} \stackrel{a}{\longrightarrow} \mathcal{D}_{2}$.

The distinguishing formula in $\mathbb{F}_{\mathrm{PML}_{\exists, \geq}}^{i+1}$ for $s_{1}$ and $s_{2}$ is then $\langle a\rangle_{p} \phi_{\mathcal{G}}$.

The proof of the second result is similar to the proof of the first one up to the use of $\geq$ in place of $\leq$ and $>$ in place of $<$ wherever necessary. 
Proof of Thm. 8. Similar to the proof of Thm. 6 - based on Lemmata 1 and 2 - up to the use of $\bigsqcup$ and $\Pi$ in place of individual values when comparing the probabilities of reaching a group of equivalence classes and the use of $\sim_{\mathrm{PB}, \mathrm{gbg}, \sqcup \sqcap}^{i}$, $\mathcal{F}_{\mathrm{PML}_{\forall, \mathrm{I}}}^{i}$, and $\mathcal{M}_{\mathrm{PML}_{\forall, \mathrm{I}}}$ in place of $\sim_{\mathrm{PB}, \mathrm{gbg},=}^{i}, \mathcal{F}_{\mathrm{PML}_{\exists, \mathrm{I}}}^{i}$, and $\mathcal{M}_{\mathrm{PML}_{\exists, \mathrm{I}}}$ wherever necessary.

In particular, for the induction step of Lemma 2 we point out that:

- In the $(\Longrightarrow)$ part, from $s_{1} \in \mathcal{M}_{\mathrm{PML}_{\forall, \mathrm{I}}} \llbracket\langle a\rangle_{\left[p_{1}, p_{2}\right]} \phi^{\prime} \rrbracket \quad$ and $s_{2} \notin \mathcal{M}_{\mathrm{PML}_{\forall, I} \mathbb{I}} \llbracket\langle a\rangle_{\left[p_{1}, p_{2}\right]} \phi^{\prime} \rrbracket$, it follows that:

- $s_{1} \stackrel{a}{\longrightarrow}$ and $p_{1} \leq \mathcal{D}_{1}\left(\mathcal{M}_{\mathrm{PML}_{\forall, \mathrm{I}}} \llbracket \phi^{\prime} \rrbracket\right) \leq p_{2}$ for all $\mathcal{D}_{1}$ such that $s_{1} \stackrel{a}{\longrightarrow} \mathcal{D}_{1}$.

- $s_{2} \stackrel{a}{\rightarrow}$ or $\mathcal{D}_{2}\left(\mathcal{M}_{\mathrm{PML}_{\forall, \mathrm{I}}} \llbracket \phi^{\prime} \rrbracket\right)<p_{1}$ or $\mathcal{D}_{2}\left(\mathcal{M}_{\mathrm{PML}_{\forall, \mathrm{I}}} \llbracket \phi^{\prime} \rrbracket\right)>p_{2}$ for some $\mathcal{D}_{2}$ such that $s_{2} \stackrel{a}{\longrightarrow} \mathcal{D}_{2}$.

Since $\phi^{\prime} \in \mathbb{F}_{\mathrm{PML}}^{i}$, by the induction hypothesis there exists $\mathcal{G} \in 2^{S / \sim_{\mathrm{PB}, \mathrm{gbg}, \text { பп }}^{i}}$ such that $\bigcup_{C \in \mathcal{G}} C=\mathcal{M}_{\mathrm{PML}_{\forall, \mathrm{I}}} \llbracket \phi^{\prime} \rrbracket$. Then:

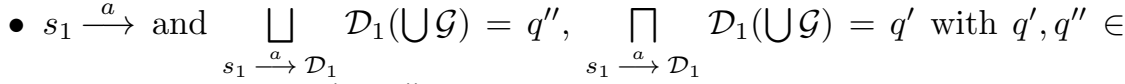
$\mathbb{R}_{\left[p_{1}, p_{2}\right]}$ such that $q^{\prime} \leq q^{\prime \prime}$.

- $s_{2} \stackrel{q}{\longrightarrow}$ or $\underset{s_{2} \stackrel{\bigsqcup^{\longrightarrow}}{\longrightarrow} \mathcal{D}_{2}}{ } \mathcal{D}_{2}(\bigcup \mathcal{G})>q^{\prime \prime}$ or $\prod_{s_{2} \stackrel{a}{\longrightarrow} \mathcal{D}_{2}} \mathcal{D}_{2}(\bigcup \mathcal{G})<q^{\prime}$.

- In the $(\Longleftarrow)$ part, if $s_{1} \sim_{\mathrm{PB}, \mathrm{gbg}, \sqcup \sqcap}^{i} s_{2}$, then there exist $p_{1}^{\prime}, p_{1}^{\prime \prime}, p_{2}^{\prime}, p_{2}^{\prime \prime} \in \mathbb{R}_{[0,1]}$ - with $p_{1}^{\prime} \leq p_{1}^{\prime \prime}$ and $p_{2}^{\prime} \leq p_{2}^{\prime \prime}$ - and $\mathcal{G} \in 2^{S / \sim_{\mathrm{PB}, \mathrm{gbg},}^{i} \cup \mathrm{n}}$ such that:

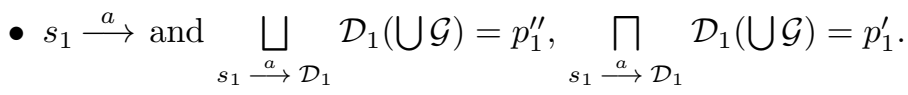

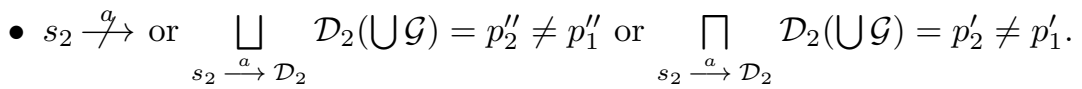

Let $\mathcal{G}_{1}=\left\{C \in S / \sim_{\mathrm{PB}, \mathrm{gbg}, \sqcup \sqcap}^{i} \mid \exists \mathcal{D}_{1} . s_{1} \stackrel{a}{\longrightarrow} \mathcal{D}_{1} \wedge \mathcal{D}_{1}(C)>0\right\}$ and $\mathcal{G}_{2}=\{C \in$ $\left.S / \sim_{\mathrm{PB}, \mathrm{gbg}, \sqcup \sqcap}^{i} \mid \exists \mathcal{D}_{2} . s_{2} \stackrel{a}{\longrightarrow} \mathcal{D}_{2} \wedge \mathcal{D}_{2}(C)>0\right\}$. The distinguishing formula in $\mathbb{F}_{\mathrm{PML} \mathrm{L}_{\forall, \mathrm{I}}}^{i+1}$ for $s_{1}$ and $s_{2}$ is then:

- $\langle a\rangle_{\left[p_{1}^{\prime}, p_{1}^{\prime \prime}\right]} \phi_{\mathcal{G}}$ if $s_{2} \stackrel{a}{\rightarrow}$ or it is not the case that $p_{1}^{\prime} \leq p_{2}^{\prime}$ and $p_{2}^{\prime \prime} \leq p_{1}^{\prime \prime}$.

- $\langle a\rangle_{\left[p_{2}^{\prime}, p_{2}^{\prime \prime}\right]} \phi_{\mathcal{G}}$ if $s_{2} \stackrel{a}{\longrightarrow}$ and it is the case that $p_{1}^{\prime} \leq p_{2}^{\prime}$ and $p_{2}^{\prime \prime} \leq p_{1}^{\prime \prime}$.

Proof of Thm. 9. The proof of the first result is similar to the proof of Thm. 6 - based on Lemmata 1 and 2 - up to the use of $\bigsqcup$ in place of individual values when comparing the probabilities of reaching a group of equivalence classes and the use of $\sim_{\mathrm{PB}, \mathrm{gbg}, \sqcup}^{i}, \mathcal{F}_{\mathrm{PML}}^{i}, \leq$ and $\mathcal{M}_{\mathrm{PML}_{\forall, \leq}}$ in place of $\sim_{\mathrm{PB}, \mathrm{gbg},=}^{i}, \mathcal{F}_{\mathrm{PML}}^{i} \mathrm{M}_{\exists, \mathrm{I}}$, and $\mathcal{M}_{\mathrm{PML}_{\exists, \mathrm{I}}}$ wherever necessary.

In particular, for the induction step of Lemma 2 we point out that:

- In the $(\Longrightarrow)$ part, from $s_{1} \in \mathcal{M}_{\mathrm{PML}_{\forall, \leq} \llbracket} \llbracket\langle a\rangle_{p} \phi^{\prime} \rrbracket \quad$ and $s_{2} \notin \mathcal{M}_{\mathrm{PML}_{\forall, \leq}} \llbracket\langle a\rangle_{p} \phi^{\prime} \rrbracket$, it follows that:

- $s_{1} \stackrel{a}{\longrightarrow}$ and $\mathcal{D}_{1}\left(\mathcal{M}_{\mathrm{PML}_{\forall}, \leq} \llbracket \phi^{\prime} \rrbracket\right) \leq p$ for all $\mathcal{D}_{1}$ such that $s_{1} \stackrel{a}{\longrightarrow} \mathcal{D}_{1}$.

- $s_{2} \stackrel{a}{\longrightarrow}$ or $\mathcal{D}_{2}\left(\mathcal{M}_{\mathrm{PML}_{\forall, \leq} \leq} \llbracket \phi^{\prime} \rrbracket\right)>p$ for some $\mathcal{D}_{2}$ such that $s_{2} \stackrel{a}{\longrightarrow} \mathcal{D}_{2}$. 
Since $\phi^{\prime} \in \mathbb{F}_{\mathrm{PML} \forall, \leq}^{i}$, by the induction hypothesis there exists $\mathcal{G} \in 2^{S / \sim_{\mathrm{PB}, \mathrm{gbg},}^{i}, \sqcup}$ such that $\bigcup_{C \in \mathcal{G}} C=\mathcal{M}_{\mathrm{PML}_{\forall}, \leq} \llbracket \phi^{\prime} \rrbracket$. Then:

$$
\begin{aligned}
& \text { - } s_{1} \stackrel{a}{\longrightarrow} \text { and } \underset{s_{1}}{\bigsqcup} \mathcal{a}_{1} \mathcal{D}_{1}(\bigcup \mathcal{G}) \leq p \text {. } \\
& \text { - } s_{2} \stackrel{a}{\longrightarrow} \text { or } \underset{s_{2} \stackrel{a}{\longrightarrow} \mathcal{D}_{2}}{\mathcal{D}_{2}(\bigcup \mathcal{G})>p .}
\end{aligned}
$$

- In the $(\Longleftarrow)$ part, if $s_{1} \sim_{\text {PB,gbg }, \sqcup}^{i} s_{2}$, then there exist $p \in \mathbb{R}_{[0,1]}$ and $\mathcal{G} \in$ $2^{S / \sim \sim_{\mathrm{PB}, \mathrm{gbg}, \sqcup}^{i}}$ such that:

- $s_{1} \stackrel{a}{\longrightarrow}$ and $\underset{s_{1} \stackrel{a}{\longrightarrow} \mathcal{D}_{1}}{\bigcup} \mathcal{D}_{1}(\bigcup \mathcal{G})=p$.

- $s_{2} \stackrel{a}{\longrightarrow}$ or $\underset{s_{2} \stackrel{a}{\longrightarrow} \mathcal{D}_{2}}{\bigsqcup} \mathcal{D}_{2}(\bigcup \mathcal{G})=q \neq p$.

Let $\mathcal{G}_{1}=\left\{C \in S / \sim_{\mathrm{PB}, \mathrm{gbg}, \sqcup}^{i} \mid \exists \mathcal{D}_{1} . s_{1} \stackrel{a}{\longrightarrow} \mathcal{D}_{1} \wedge \mathcal{D}_{1}(C)>0\right\}$ and $\mathcal{G}_{2}=\{C \in$ $\left.S / \sim_{\mathrm{PB}, \mathrm{gbg}, \sqcup} \mid \exists \mathcal{D}_{2} . s_{2} \stackrel{a}{\longrightarrow} \mathcal{D}_{2} \wedge \mathcal{D}_{2}(C)>0\right\}$. The distinguishing formula in $\mathbb{F}_{\mathrm{PML}_{\forall} \leq \leq}^{i+1}$ for $s_{1}$ and $s_{2}$ is then:

- $\langle a\rangle_{p} \phi_{\mathcal{G}}$ if $s_{2} \stackrel{q}{\longrightarrow}$ or $p<q$.

- $\langle a\rangle_{q} \phi_{\mathcal{G}}$ if $s_{2} \stackrel{a}{\longrightarrow}$ and $q<p$.

The proof of the second result is similar to the proof of the first one up to the use of $\Pi$ in place of $\sqcup, \geq$ in place of $\leq,<$ in place of $>$, and $>$ in place of $<$ wherever necessary.

Proof of Thm. 10. Let $\mathcal{U}=(S, A, \longrightarrow)$ be an NPLTS and $s_{1}, s_{2} \in S$ :

1. The fact that $s_{1} \sim_{\mathrm{PB} \text {,dis }} s_{2}$ implies $s_{1} \sim_{\mathrm{PB}, \mathrm{gbg},=} s_{2}$ is a straightforward consequence of the fact that a class-distribution probabilistic bisimulation is trivially a =-group-by-group probabilistic bisimulation.

Suppose now that $s_{1} \sim_{\mathrm{PB}, \mathrm{gbg},=} s_{2}$. This means that there exists a =-groupby-group probabilistic bisimulation $\mathcal{B}$ over $S$ such that $\left(s_{1}, s_{2}\right) \in \mathcal{B}$. In other words, whenever $\left(s_{1}^{\prime}, s_{2}^{\prime}\right) \in \mathcal{B}$, then for all $a \in A$ and $\mathcal{G} \in 2^{S / \mathcal{B}}$ :

- For each $s_{1}^{\prime} \stackrel{a}{\longrightarrow} \mathcal{D}_{1}$ there exists $s_{2}^{\prime} \stackrel{a}{\longrightarrow} \mathcal{D}_{2}$ such that $\mathcal{D}_{1}(\bigcup \mathcal{G})=\mathcal{D}_{2}(\bigcup \mathcal{G})$.

- For each $s_{2}^{\prime} \stackrel{a}{\longrightarrow} \mathcal{D}_{2}$ there exists $s_{1}^{\prime} \stackrel{a}{\longrightarrow} \mathcal{D}_{1}$ such that $\mathcal{D}_{2}(\bigcup \mathcal{G})=\mathcal{D}_{1}(\bigcup \mathcal{G})$. This means that, whenever $\left(s_{1}^{\prime}, s_{2}^{\prime}\right) \in \mathcal{B}$, then for all $a \in A$ and $\mathcal{G} \in 2^{S / \mathcal{B}}$ :

$$
\begin{aligned}
& \text { - If } s_{1}^{\prime} \stackrel{a}{\longrightarrow} \text {, then } s_{2}^{\prime} \stackrel{a}{\longrightarrow} \text { with } \underset{s_{1}^{\prime} \stackrel{a}{\longrightarrow} \mathcal{D}_{1}}{\bigcup}\left\{\mathcal{D}_{1}(\bigcup \mathcal{G})\right\} \subseteq \underset{s_{2}^{\prime} \stackrel{a}{\longrightarrow} \mathcal{D}_{2}}{\bigcup}\left\{\mathcal{D}_{2}(\bigcup \mathcal{G})\right\} \text {. } \\
& \text { - If } s_{2}^{\prime} \stackrel{a}{\longrightarrow} \text {, then } s_{1}^{\prime} \stackrel{a}{\longrightarrow} \text { with } \underset{s_{2}^{\prime} \stackrel{a}{\longrightarrow} \mathcal{D}_{2}}{\bigcup}\left\{\mathcal{D}_{2}(\bigcup \mathcal{G})\right\} \subseteq \underset{s_{1}^{\prime} \stackrel{a}{\longrightarrow} \mathcal{D}_{1}}{\bigcup}\left\{\mathcal{D}_{1}(\bigcup \mathcal{G})\right\} \text {. }
\end{aligned}
$$

Equivalently, if both $s_{1}^{\prime}$ and $s_{2}^{\prime}$ have at least one outgoing $a$-transition, then:

and hence:

$$
\bigcup_{s_{1}^{\prime} \stackrel{a}{\longrightarrow} \mathcal{D}_{1}}\left\{\mathcal{D}_{1}(\bigcup \mathcal{G})\right\}=\underset{s_{2}^{\prime} \stackrel{a}{\longrightarrow} \mathcal{D}_{2}}{\bigcup}\left\{\mathcal{D}_{2}(\bigcup \mathcal{G})\right\}
$$

$$
\begin{aligned}
& \bigsqcup_{s_{1}^{\prime} \stackrel{\bigsqcup_{a}}{\longrightarrow} \mathcal{D}_{1}} \mathcal{D}_{1}(\bigcup \mathcal{G})=\bigsqcup_{s_{2}^{\prime} \stackrel{\bigsqcup_{a}}{\longrightarrow} \mathcal{D}_{2}} \mathcal{D}_{2}(\bigcup \mathcal{G}) \\
& \prod_{s_{1}^{\prime} \stackrel{a}{\longrightarrow} \mathcal{D}_{1}} \mathcal{D}_{1}(\bigcup \mathcal{G})=\prod_{s_{2}^{\prime} \stackrel{a}{\longrightarrow} \mathcal{D}_{2}} \mathcal{D}_{2}(\bigcup \mathcal{G})
\end{aligned}
$$

Therefore, $\mathcal{B}$ is also a $\sqcup П$-group-by-group probabilistic bisimulation, i.e., $s_{1} \sim_{\mathrm{PB}, \mathrm{gbg}, \sqcup \sqcap} s_{2}$. 
2. Suppose that $s_{1} \sim_{\mathrm{PB}, \mathrm{gbg}, \leq} \leq s_{2}$. This means that there exists a $\leq$-group-bygroup probabilistic bisimulation $\mathcal{B}$ over $S$ such that $\left(s_{1}, s_{2}\right) \in \mathcal{B}$. In other words, whenever $\left(s_{1}^{\prime}, s_{2}^{\prime}\right) \in \mathcal{B}$, then for all $a \in A$ and $\mathcal{G} \in 2^{S / \mathcal{B}}$ :

- For each $s_{1}^{\prime} \stackrel{a}{\longrightarrow} \mathcal{D}_{1}$ there exists $s_{2}^{\prime} \stackrel{a}{\longrightarrow} \mathcal{D}_{2}$ such that $\mathcal{D}_{1}(\bigcup \mathcal{G}) \leq \mathcal{D}_{2}(\bigcup \mathcal{G})$.

- For each $s_{2}^{\prime} \stackrel{a}{\longrightarrow} \mathcal{D}_{2}$ there exists $s_{1}^{\prime} \stackrel{a}{\longrightarrow} \mathcal{D}_{1}$ such that $\mathcal{D}_{2}(\bigcup \mathcal{G}) \leq \mathcal{D}_{1}(\bigcup \mathcal{G})$. This means that, whenever $\left(s_{1}^{\prime}, s_{2}^{\prime}\right) \in \mathcal{B}$, then for all $a \in A$ and $\mathcal{G} \in 2^{S / \mathcal{B}}$ :

$$
\begin{aligned}
& \text { - If } s_{1}^{\prime} \stackrel{a}{\longrightarrow} \text {, then } s_{2}^{\prime} \stackrel{a}{\longrightarrow} \text { with } \underset{s_{1}^{\prime} \stackrel{a}{\longrightarrow} \mathcal{D}_{1}}{\bigsqcup} \mathcal{D}_{1}(\cup \mathcal{G}) \leq \underset{s_{2}^{\prime} \stackrel{a}{\longrightarrow} \mathcal{D}_{2}}{\bigsqcup_{2}} \mathcal{D}_{2}(\cup \mathcal{G}) \text {. } \\
& \text { - If } s_{2}^{\prime} \stackrel{a}{\longrightarrow} \text {, then } s_{1}^{\prime} \stackrel{a}{\longrightarrow} \text { with } \underset{s_{2}^{\prime} \stackrel{a}{\longrightarrow} \mathcal{D}_{2}}{\bigsqcup} \mathcal{D}_{2}(\cup \mathcal{G}) \leq \underset{s_{1}^{\prime} \stackrel{a}{\longrightarrow} \mathcal{D}_{1}}{\bigsqcup} \mathcal{D}_{1}(\cup \mathcal{G}) \text {. }
\end{aligned}
$$

Equivalently, if both $s_{1}^{\prime}$ and $s_{2}^{\prime}$ have at least one outgoing $a$-transition, then:

$$
\stackrel{\bigsqcup}{s_{1}^{\prime} \stackrel{a}{\longrightarrow} \mathcal{D}_{1}} \mathcal{D}_{1}(\bigcup \mathcal{G})=\bigsqcup_{s_{2}^{\prime} \stackrel{\bigsqcup}{\longrightarrow} \mathcal{D}_{2}} \mathcal{D}_{2}(\bigcup \mathcal{G})
$$

Therefore, $\mathcal{B}$ is also a $\sqcup$-group-by-group probabilistic bisimulation, i.e., $s_{1} \sim$ PB,gbg, $\sqcup s_{2}$.

The reverse implication holds too when the NPLTS $\mathcal{U}$ is image finite. In fact, this property guarantees that the following two sets:

$$
\underset{s_{1}^{\prime} \stackrel{\bigcup_{a}}{\longrightarrow} \mathcal{D}_{1}}{\bigcup}\left\{\mathcal{D}_{1}(\bigcup \mathcal{G})\right\} \quad \text { and } \underset{s_{2}^{\prime} \stackrel{a}{\longrightarrow} \mathcal{D}_{2}}{\bigcup}\left\{\mathcal{D}_{2}(\bigcup \mathcal{G})\right\}
$$

are finite. In turn, the finiteness of those two sets ensures that their suprema respectively belong to the two sets themselves. As a consequence, starting from:

or equivalently:

$$
\underset{s_{1}^{\prime} \stackrel{\bigsqcup_{a}}{\longrightarrow} \mathcal{D}_{1}}{ } \mathcal{D}_{1}(\bigcup \mathcal{G})=\bigsqcup_{s_{2}^{\prime} \stackrel{\bigsqcup^{\longrightarrow}}{\longrightarrow} \mathcal{D}_{2}} \mathcal{D}_{2}(\bigcup \mathcal{G})
$$

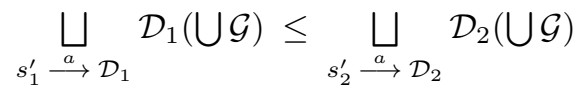

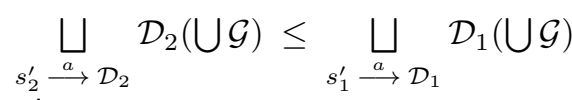

when both $s_{1}^{\prime}$ and $s_{2}^{\prime}$ have at least one outgoing $a$-transition, the following holds:

- If $s_{1}^{\prime} \stackrel{a}{\longrightarrow} \mathcal{D}_{1}^{\prime}$, then $s_{2}^{\prime} \stackrel{a}{\longrightarrow} \mathcal{D}_{2}^{\prime}$ with $\mathcal{D}_{1}^{\prime}(\cup \mathcal{G}) \leq \mathcal{D}_{2}^{\prime}(\bigcup \mathcal{G})$ because we can

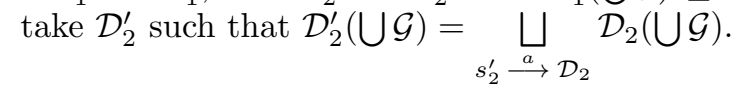

- If $s_{2}^{\prime} \stackrel{a}{\longrightarrow} \mathcal{D}_{2}^{\prime}$, then $s_{1}^{\prime} \stackrel{a}{\longrightarrow} \mathcal{D}_{1}^{\prime}$ with $\mathcal{D}_{2}^{\prime}(\cup \mathcal{G}) \leq \mathcal{D}_{1}^{\prime}(\bigcup \mathcal{G})$ because we can

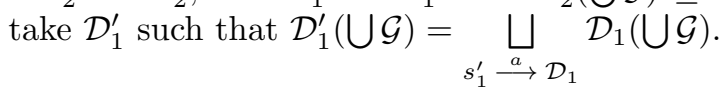

3. Similar to the previous proof up to the use of $\geq$ in place of $\leq$ and $\sqcap$ in place of $\sqcup$ wherever necessary.

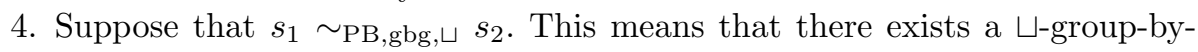
group probabilistic bisimulation $\mathcal{B}$ over $S$ such that $\left(s_{1}, s_{2}\right) \in \mathcal{B}$. In other words, whenever $\left(s_{1}^{\prime}, s_{2}^{\prime}\right) \in \mathcal{B}$, then for all $a \in A$ and $\mathcal{G} \in 2^{S / \mathcal{B}}$ it holds that $s_{1}^{\prime} \stackrel{a}{\longrightarrow}$ implies $s_{2}^{\prime} \stackrel{a}{\longrightarrow}$ with:

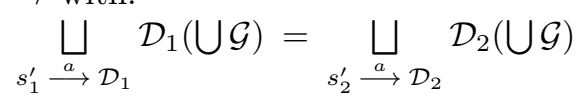


Then $\mathcal{B}$ must be a $\sqcap$-group-by-group probabilistic bisimulation as well and hence $s_{1} \sim \mathrm{PB}, \mathrm{gbg}, \sqcap s_{2}$. In fact, if this were not the case, then there would exist $a^{\prime} \in A$ and $\mathcal{G}^{\prime} \in 2^{S / \mathcal{B}}$ such that $s_{1}^{\prime} \stackrel{a^{\prime}}{\longrightarrow}, s_{2}^{\prime} \stackrel{a}{\longrightarrow}$, and:

$$
\prod_{s_{1}^{\prime} \stackrel{a^{\prime}}{\longrightarrow} \mathcal{D}_{1}} \mathcal{D}_{1}\left(\bigcup \mathcal{G}^{\prime}\right) \neq \prod_{s_{2}^{\prime} \stackrel{a^{\prime}}{\longrightarrow} \mathcal{D}_{2}} \mathcal{D}_{2}\left(\bigcup \mathcal{G}^{\prime}\right)
$$

As a consequence, denoting by $\mathcal{G}^{\prime \prime}$ the group of all the equivalence classes not in $\mathcal{G}^{\prime}$, it would hold that $s_{1}^{\prime} \stackrel{a^{\prime}}{\longrightarrow}, s_{2}^{\prime} \stackrel{a}{\longrightarrow}$, and:

$$
\begin{aligned}
& \bigsqcup \mathcal{D}_{1}\left(\bigcup \mathcal{G}^{\prime \prime}\right)=1-\sqcap \mathcal{D}_{1}\left(\bigcup \mathcal{G}^{\prime}\right) \\
& s_{1}^{\prime} \stackrel{a^{\prime}}{\longrightarrow} \mathcal{D}_{1} \\
& s_{1}^{\prime} \stackrel{a^{\prime}}{\longrightarrow} \mathcal{D}_{1} \\
& \neq 1-\sqcap \mathcal{D}_{2}\left(\bigcup \mathcal{G}^{\prime}\right) \\
& =\underset{s_{2}^{\prime} \stackrel{a^{\prime}}{\longrightarrow} \mathcal{D}_{2}}{\stackrel{s_{2}^{\prime}}{\stackrel{a^{\prime}}{\longrightarrow}} \mathcal{D}_{2}} \mathcal{D}_{2}\left(\bigcup \mathcal{G}^{\prime \prime}\right)
\end{aligned}
$$

thus contradicting the fact that $\mathcal{B}$ is a $\sqcup$-group-by-group probabilistic bisimulation.

By proceeding in a similar way, we can prove that $s_{1} \sim_{\mathrm{PB}, \mathrm{gbg}, \sqcap} s_{2}$ implies $s_{1} \sim_{\mathrm{PB}, \mathrm{gbg}, \sqcup} s_{2}$. Therefore, $\sim_{\mathrm{PB}, \mathrm{gbg}, \sqcup}$ and $\sim_{\mathrm{PB}, \mathrm{gbg}, \sqcap}$ coincide.

Finally, we prove that $\sim_{\mathrm{PB}, \mathrm{gbg}, \sqcup \sqcap}$ and $\sim_{\mathrm{PB}, \mathrm{gbg}, \sqcup}$ coincide. If $s_{1} \sim_{\mathrm{PB}, \mathrm{gbg}, \sqcup \sqcap} s_{2}$, then $s_{1} \sim$ PB,gbg, $\sqcup s_{2}$ because a $\sqcup \sqcap$-group-by-group probabilistic bisimulation is trivially a $\sqcup$-group-by-group probabilistic bisimulation. Suppose now that

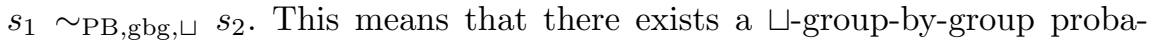
bilistic bisimulation $\mathcal{B}$ over $S$ such that $\left(s_{1}, s_{2}\right) \in \mathcal{B}$. Since $\mathcal{B}$ must also be a $\sqcap$-group-by-group probabilistic bisimulation, whenever $\left(s_{1}^{\prime}, s_{2}^{\prime}\right) \in \mathcal{B}$, then for all $a \in A$ and $\mathcal{G} \in 2^{S / \mathcal{B}}$ it holds that $s_{1}^{\prime} \stackrel{a}{\longrightarrow}$ implies $s_{2}^{\prime} \stackrel{a}{\longrightarrow}$ with:

$$
\begin{aligned}
& \bigsqcup_{s^{\prime}} \mathcal{D}_{1}(\bigcup \mathcal{G})=\bigsqcup_{s^{\prime}} \mathcal{D}_{2}(\bigcup \mathcal{G})
\end{aligned}
$$

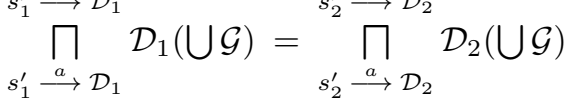

This means that $\mathcal{B}$ is also a $\sqcup П$-group-by-group probabilistic bisimulation, i.e., $s_{1} \sim$ PB,gbg, $\sqcup \sqcap s_{2}$.

Proof of Thm. 11. Let $\mathcal{U}=(S, A, \longrightarrow)$ be an NPLTS and $s_{1}, s_{2} \in S$ :

1. Since an ordinary transition is a special case of combined transition in which a single transition is taken with weight 1 , it trivially holds that (i) a classdistribution probabilistic bisimulation is a class-distribution ct-probabilistic bisimulation and (ii) a =-group-by-group probabilistic bisimulation is a =-group-by-group ct-probabilistic bisimulation.

2. The inclusion of $\sim_{\mathrm{PB}, \mathrm{gbg}, \triangleright}$ in $\sim_{\mathrm{PB}, \mathrm{gbg}, \triangleright}^{\mathrm{ct}}$ is a straightforward consequence of the fact that an ordinary transition is a special case of combined transition in which a single transition is taken with weight 1 . We now prove the reverse inclusions:

- Suppose that $s_{1} \sim_{\mathrm{PB}, \mathrm{gbg}, \leq}^{\mathrm{ct}} s_{2}$. This means that there exists a $\leq$-groupby-group ct-probabilistic bisimulation $\mathcal{B}$ over $S$ such that $\left(s_{1}, s_{2}\right) \in \mathcal{B}$. In other words, whenever $\left(s_{1}^{\prime}, s_{2}^{\prime}\right) \in \mathcal{B}$, then for all $a \in A$ and $\mathcal{G} \in$ 
$2^{S / \mathcal{B}}$ it holds that for each $s_{1}^{\prime} \stackrel{a}{\longrightarrow} \mathcal{D}_{1}$ there exists $s_{2}^{\prime} \stackrel{a}{\longrightarrow}{ }_{\mathrm{c}} \mathcal{D}_{2}$ such that $\mathcal{D}_{1}(\bigcup \mathcal{G}) \leq \mathcal{D}_{2}(\bigcup \mathcal{G})$. On the side of $s_{2}^{\prime}$, this means that there exist $n \in \mathbb{N}_{>0},\left\{p_{i} \in \mathbb{R}_{] 0,1]} \mid 1 \leq i \leq n\right\}$, and $\left\{s_{2}^{\prime} \stackrel{a}{\longrightarrow} \mathcal{D}_{2, i} \mid 1 \leq i \leq n\right\}$ such that $\sum_{i=1}^{n} p_{i}=1$ and $\sum_{i=1}^{n} p_{i} \cdot \mathcal{D}_{2, i}=\mathcal{D}_{2}$. As a consequence:

$$
\begin{aligned}
\mathcal{D}_{2}(\bigcup \mathcal{G}) & \leq \sum_{i=1}^{n} p_{i} \cdot \max _{1 \leq i \leq n} \mathcal{D}_{2, i}(\bigcup \mathcal{G}) \\
& =\max _{1 \leq i \leq n} \mathcal{D}_{2, i}(\bigcup \mathcal{G}) \cdot \sum_{i=1}^{n} p_{i} \\
& =\max _{1 \leq i \leq n} \mathcal{D}_{2, i}(\bigcup \mathcal{G})
\end{aligned}
$$

and hence there exists $\mathcal{D}_{2}^{\prime}$ such that $s_{2}^{\prime} \stackrel{a}{\longrightarrow} \mathcal{D}_{2}^{\prime}$ with $\mathcal{D}_{1}(\bigcup \mathcal{G}) \leq \mathcal{D}_{2}^{\prime}(\bigcup \mathcal{G})$. This means that $\mathcal{B}$ is also a $\leq$-group-by-group probabilistic bisimulation, i.e., $s_{1} \sim_{\mathrm{PB}, \mathrm{gbg}, \leq} s_{2}$.

- The proof that $s_{1} \sim_{\mathrm{PB}, \mathrm{gbg}, \geq}^{\mathrm{ct}}, s_{2} \Longrightarrow s_{1} \sim_{\mathrm{PB}, \mathrm{gbg}, \geq} s_{2}$ is similar to the previous proof up to the use of $\geq$ in place of $\leq$ and min in place of max wherever necessary.

- Suppose that $s_{1} \sim_{\mathrm{PB}, \mathrm{gbg}, \sqcup \sqcap}^{\mathrm{ct}} s_{2}$. This means that there exists a பП-groupby-group ct-probabilistic bisimulation $\mathcal{B}$ over $S$ such that $\left(s_{1}, s_{2}\right) \in \mathcal{B}$. In other words, whenever $\left(s_{1}^{\prime}, s_{2}^{\prime}\right) \in \mathcal{B}$, then for all $a \in A$ and $\mathcal{G} \in 2^{S / \mathcal{B}}$ it holds that $s_{1}^{\prime} \stackrel{a}{\longrightarrow}$ implies $s_{2}^{\prime} \stackrel{a}{\longrightarrow}$ with:

$$
\begin{aligned}
& \bigsqcup_{s_{1}^{\prime} \stackrel{a}{\longrightarrow}{ }_{\mathrm{c}} \mathcal{D}_{1}} \mathcal{D}_{1}(\bigcup \mathcal{G})=\bigsqcup_{s_{2}^{\prime} \stackrel{a}{\longrightarrow}{ }_{\mathrm{c}} \mathcal{D}_{2}} \mathcal{D}_{2}(\bigcup \mathcal{G}) \\
& \prod_{s_{1}^{\prime} \stackrel{a}{\longrightarrow} \mathcal{D}_{\mathrm{c}}} \mathcal{D}_{1}(\bigcup \mathcal{G})=\prod_{s_{2}^{\prime} \stackrel{a}{\longrightarrow}{ }_{\mathrm{c}} \mathcal{D}_{2}} \mathcal{D}_{2}(\bigcup \mathcal{G})
\end{aligned}
$$

Given $a \in A, \mathcal{G} \in 2^{S / \mathcal{B}}$, and $s \in S$ having at least one outgoing $a$-transition, when $\mathcal{U}$ is image finite it holds that:

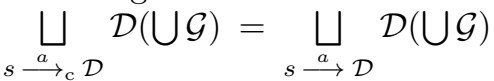

$$
\begin{aligned}
& \prod_{s \stackrel{a}{\longrightarrow} \mathcal{D}} \mathcal{D}(\bigcup \mathcal{G})=\prod_{s \stackrel{a}{\longrightarrow} \mathcal{D}} \mathcal{D}(\bigcup \mathcal{G})
\end{aligned}
$$

because the supremum and the infimum on the left are respectively achieved by two ordinary $a$-transitions of $s$. In fact, let $\mathcal{D}_{\sqcup}\left(\right.$ resp. $\left.\mathcal{D}_{\sqcap}\right)$ be the target of an $a$-transition of $s$ assigning the maximum (resp. minimum) value to $\bigcup \mathcal{G}$ among all the $a$-transitions of $s$ and consider an arbitrary convex combination of a subset $\left\{s \stackrel{a}{\longrightarrow} \mathcal{D}_{i} \mid 1 \leq i \leq n\right\}$ of those transitions, with weights $p_{1}, \ldots, p_{n}$ and $n \in \mathbb{N}_{>0}$. Then:

$$
\begin{aligned}
& \sum_{i=1}^{n} p_{i} \cdot \mathcal{D}_{i}(\bigcup \mathcal{G}) \leq \sum_{i=1}^{n} p_{i} \cdot \mathcal{D}_{\sqcup}(\bigcup \mathcal{G})=\mathcal{D}_{\sqcup}(\bigcup \mathcal{G}) \\
& \sum_{i=1}^{n} p_{i} \cdot \mathcal{D}_{i}(\bigcup \mathcal{G}) \geq \sum_{i=1}^{n} p_{i} \cdot \mathcal{D}_{\sqcap}(\bigcup \mathcal{G})=\mathcal{D}_{\sqcap}(\bigcup \mathcal{G})
\end{aligned}
$$

As a consequence, whenever $\left(s_{1}^{\prime}, s_{2}^{\prime}\right) \in \mathcal{B}$, then for all $a \in A$ and $\mathcal{G} \in 2^{S / \mathcal{B}}$ it holds that $s_{1}^{\prime} \stackrel{a}{\longrightarrow}$ implies $s_{2}^{\prime} \stackrel{a}{\longrightarrow}$ with:

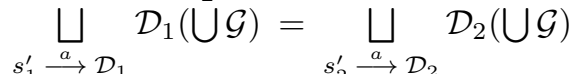

$$
\begin{aligned}
& \prod_{s_{1}^{\prime} \stackrel{a}{\longrightarrow} \mathcal{D}_{1}} \mathcal{D}_{1}(\bigcup \mathcal{G})=\prod_{s_{2}^{\prime} \stackrel{a}{\longrightarrow} \mathcal{D}_{2}} \mathcal{D}_{2}(\bigcup \mathcal{G})
\end{aligned}
$$


This means that $\mathcal{B}$ is also a $\sqcup П$-group-by-group probabilistic bisimulation, i.e., $s_{1} \sim_{\mathrm{PB}, \mathrm{gbg}, \sqcup \sqcap} s_{2}$.

- The proof that $s_{1} \sim_{\mathrm{PB}, \mathrm{gbg}, \sqcup}^{\mathrm{ct}} \sqcup s_{2} \Longrightarrow s_{1} \sim_{\mathrm{PB}, \mathrm{gbg}, \sqcup} s_{2}$ is similar to the proof that $s_{1} \sim_{\mathrm{PB}, \mathrm{gbg}, \sqcup \sqcap}^{\mathrm{ct}} s_{2} \Longrightarrow s_{1} \sim \sim_{\mathrm{PB}, \mathrm{gbg}, \sqcup \sqcap} s_{2}$.

- The proof that $s_{1} \sim_{\mathrm{PB}, \mathrm{gbg}, \sqcap}^{\mathrm{ct}} s_{2} \Longrightarrow s_{1} \sim_{\mathrm{PB}, \mathrm{gbg}, \sqcap} s_{2}$ is similar to the proof that $s_{1} \sim_{\mathrm{PB}, \mathrm{gbg}, \sqcup \sqcap}^{\mathrm{ct}} s_{2} \Longrightarrow s_{1} \sim \sim_{\mathrm{PB}, \mathrm{gbg}, \sqcup \sqcap} s_{2}$.

3. The fact that $s_{1} \sim_{\mathrm{PB}, \mathrm{dis}}^{\mathrm{ct}} s_{2}$ implies $s_{1} \sim_{\mathrm{PB}, \mathrm{gbg},=}^{\mathrm{ct}} s_{2}$ is a straightforward consequence of the fact that a class-distribution ct-probabilistic bisimulation is trivially a $=$-group-by-group ct-probabilistic bisimulation.

4. Suppose that $s_{1} \sim \sim_{\mathrm{PB}, \mathrm{ct} g}^{\mathrm{ct}}=s_{2}$. This means that there exists a =-group-bygroup ct-probabilistic bisimulation $\mathcal{B}$ over $S$ such that $\left(s_{1}, s_{2}\right) \in \mathcal{B}$. In other words, whenever $\left(s_{1}^{\prime}, s_{2}^{\prime}\right) \in \mathcal{B}$, then for all $a \in A$ and $\mathcal{G} \in 2^{S / \mathcal{B}}$ :

- For each $s_{1}^{\prime} \stackrel{a}{\longrightarrow} \mathcal{D}_{1}$ there exists $s_{2}^{\prime} \stackrel{a}{\longrightarrow}_{\mathrm{c}} \mathcal{D}_{2}$ such that $\mathcal{D}_{1}(\bigcup \mathcal{G})=\mathcal{D}_{2}(\bigcup \mathcal{G})$.

- For each $s_{2}^{\prime} \stackrel{a}{\longrightarrow} \mathcal{D}_{2}$ there exists $s_{1}^{\prime} \stackrel{a}{\longrightarrow} \mathcal{D}_{1}$ such that $\mathcal{D}_{2}(\bigcup \mathcal{G})=\mathcal{D}_{1}(\bigcup \mathcal{G})$. This implies that, whenever $\left(s_{1}^{\prime}, s_{2}^{\prime}\right) \in \mathcal{B}$, then for all $a \in A$ and $\mathcal{G} \in 2^{S / \mathcal{B}}$ :

- For each $s_{1}^{\prime} \stackrel{a}{\longrightarrow}{ }_{\mathrm{c}} \mathcal{D}_{1}$ there exists $s_{2}^{\prime} \stackrel{a}{\longrightarrow}_{\mathrm{c}} \mathcal{D}_{2}$ such that $\mathcal{D}_{1}(\bigcup \mathcal{G})=\mathcal{D}_{2}(\bigcup \mathcal{G})$.

- For each $s_{2}^{\prime} \stackrel{a}{\longrightarrow}{ }_{\mathrm{c}} \mathcal{D}_{2}$ there exists $s_{1}^{\prime} \stackrel{{ }^{a}}{\longrightarrow}{ }_{\mathrm{c}} \mathcal{D}_{1}$ such that $\mathcal{D}_{2}(\bigcup \mathcal{G})=\mathcal{D}_{1}(\bigcup \mathcal{G})$.

This means that, whenever $\left(s_{1}^{\prime}, s_{2}^{\prime}\right) \in \mathcal{B}$, then for all $a \in A$ and $\mathcal{G} \in 2^{S / \mathcal{B}}$ :

$$
\begin{aligned}
& \text { - If } s_{1}^{\prime} \stackrel{a}{\longrightarrow} \text {, then } s_{2}^{\prime} \stackrel{a}{\longrightarrow} \text { with } \underset{s_{1}^{\prime} \stackrel{a}{\longrightarrow} \bigcup_{\mathrm{c}} \mathcal{D}_{1}}{\bigcup}\left\{\mathcal{D}_{1}(\bigcup \mathcal{G})\right\} \subseteq \underset{s_{2}^{\prime} \stackrel{a}{\longrightarrow} \bigcup_{\mathrm{c}} \mathcal{D}_{2}}{\bigcup}\left\{\mathcal{D}_{2}(\bigcup \mathcal{G})\right\} \text {. } \\
& \text { - If } s_{2}^{\prime} \stackrel{a}{\longrightarrow} \text {, then } s_{1}^{\prime} \stackrel{a}{\longrightarrow} \text { with } \underset{s_{2}^{\prime} \stackrel{a}{\longrightarrow} \bigcup_{\mathrm{c}} \mathcal{D}_{2}}{\bigcup}\left\{\mathcal{D}_{2}(\bigcup \mathcal{G})\right\} \subseteq \underset{s_{1}^{\prime} \stackrel{a}{\longrightarrow}{ }_{\mathrm{c}} \mathcal{D}_{1}}{\bigcup}\left\{\mathcal{D}_{1}(\bigcup \mathcal{G})\right\} \text {. }
\end{aligned}
$$

Equivalently, if both $s_{1}^{\prime}$ and $s_{2}^{\prime}$ have at least one outgoing $a$-transition, then:

and hence:

$$
\underset{s_{1}^{\prime} \stackrel{a}{\longrightarrow} \mathcal{D}_{1}}{\bigcup}\left\{\mathcal{D}_{1}(\bigcup \mathcal{G})\right\}=\underset{s_{2}^{\prime} \stackrel{a}{\longrightarrow} \bigcup_{\mathrm{c}} \mathcal{D}_{2}}{\bigcup}\left\{\mathcal{D}_{2}(\bigcup \mathcal{G})\right\}
$$

$$
\begin{aligned}
& \bigsqcup_{s_{1}^{\prime} \stackrel{a}{\longrightarrow} \mathcal{D}_{c} \mathcal{D}_{1}} \mathcal{D}_{1}(\bigcup \mathcal{G})=\bigsqcup_{s_{2}^{\prime} \underset{a}{\longrightarrow} \mathcal{D}_{c} \mathcal{D}_{2}} \mathcal{D}_{2}(\bigcup \mathcal{G}) \\
& \prod_{s_{1}^{\prime} \stackrel{a}{\longrightarrow} \mathcal{c}_{\mathrm{c}} \mathcal{D}_{1}} \mathcal{D}_{1}(\bigcup \mathcal{G})=\prod_{s_{2}^{\prime} \stackrel{\prod_{a}}{\longrightarrow} \mathcal{D}_{\mathrm{c}} \mathcal{D}_{2}} \mathcal{D}_{2}(\bigcup \mathcal{G})
\end{aligned}
$$

Therefore, $\mathcal{B}$ is also a $\sqcup \sqcap$-group-by-group ct-probabilistic bisimulation, i.e., $s_{1} \sim_{\mathrm{PB}, \mathrm{gbg}, \sqcup \sqcap}^{\mathrm{ct}} s_{2}$.

Suppose now that $s_{1} \sim_{\mathrm{PB}, \mathrm{gbg}, \sqcup \sqcap}^{\mathrm{ct}} s_{2}$. This means that there exists a பПgroup-by-group ct-probabilistic bisimulation $\mathcal{B}$ over $S$ such that $\left(s_{1}, s_{2}\right) \in \mathcal{B}$. Given $a \in A$ and $\mathcal{G} \in 2^{S / \mathcal{B}}$, assume that there exists $s_{1} \stackrel{a}{\longrightarrow} \mathcal{D}_{1}$ such that $\mathcal{D}_{1}(\bigcup \mathcal{G})=p$. Since $\left(s_{1}, s_{2}\right) \in \mathcal{B}$ and the NPLTS is image finite, there exist $s_{2} \stackrel{a}{\longrightarrow}_{\mathrm{c}} \mathcal{D}_{2}^{\prime}$ such that $\mathcal{D}_{2}^{\prime}(\bigcup \mathcal{G})=p^{\prime} \leq p$ and $s_{2}{ }_{a^{a}}{ }_{\mathrm{c}} \mathcal{D}_{2}^{\prime \prime}$ such that $\mathcal{D}_{2}^{\prime \prime}(\bigcup \mathcal{G})=p^{\prime \prime} \geq p$. If $p^{\prime}=p$ (resp. $p^{\prime \prime}=p$ ), then $s_{1} \stackrel{a}{\longrightarrow} \mathcal{D}_{1}$ is trivially matched by $s_{2} \stackrel{a}{\longrightarrow} \mathcal{D}_{2}^{\prime}$ (resp. $s_{2} \stackrel{a}{\longrightarrow} \mathcal{D}_{2}^{\prime \prime}$ ) with respect to $\sim_{\mathrm{PB}, \mathrm{gbg},=}^{\mathrm{ct}}$ when considering $\mathcal{G}$.

Assume that $p^{\prime}<p<p^{\prime \prime}$ and note that $s_{2} \stackrel{a}{\longrightarrow}_{\mathrm{c}}\left(x \cdot \mathcal{D}_{2}^{\prime}+y \cdot \mathcal{D}_{2}^{\prime \prime}\right)$ for all $x, y \in \mathbb{R}_{0,1]}$ such that $x+y=1$. Indeed, directly from the definition of combined transition, we have that: 
- Since $s_{2} \stackrel{a}{\longrightarrow} \mathcal{D}_{2}^{\prime}$, there exist $n \in \mathbb{N}_{>0},\left\{p_{i}^{\prime} \in \mathbb{R}_{0,1]} \mid 1 \leq i \leq n\right\}$, and $\left\{s_{2} \stackrel{a}{\longrightarrow} \hat{\mathcal{D}}_{i}^{\prime} \mid 1 \leq i \leq n\right\}$ such that $\sum_{i=1}^{n} p_{i}^{\prime}=1$ and $\sum_{i=1}^{n} p_{i}^{\prime} \cdot \hat{\mathcal{D}}_{i}^{\prime}=\mathcal{D}_{2}^{\prime}$.

- Since $s_{2} \stackrel{a}{\longrightarrow}_{\mathrm{c}} \mathcal{D}_{2}^{\prime \prime}$, there exist $m \in \mathbb{N}_{>0},\left\{p_{j}^{\prime \prime} \in \mathbb{R}_{00,1]} \mid 1 \leq j \leq m\right\}$, and $\left\{s_{2} \stackrel{a}{\longrightarrow} \hat{\mathcal{D}}_{j}^{\prime \prime} \mid 1 \leq j \leq m\right\}$ such that $\sum_{j=1}^{m} p_{j}^{\prime \prime}=1$ and $\sum_{j=1}^{m} p_{j}^{\prime \prime} \cdot \hat{\mathcal{D}}_{j}^{\prime \prime}=\mathcal{D}_{2}^{\prime \prime}$. Hence, $\left(x \cdot \mathcal{D}_{2}^{\prime}+y \cdot \mathcal{D}_{2}^{\prime \prime}\right)$ can be obtained from the appropriate combination of:

with coefficients:

$$
\left\{s_{2} \stackrel{a}{\longrightarrow} \hat{\mathcal{D}}_{i}^{\prime} \mid 1 \leq i \leq n\right\} \cup\left\{s_{2} \stackrel{a}{\longrightarrow} \hat{\mathcal{D}}_{j}^{\prime \prime} \mid 1 \leq j \leq m\right\}
$$

$$
\left\{x \cdot p_{i}^{\prime} \in \mathbb{R}_{0,1]} \mid 1 \leq i \leq n\right\} \cup\left\{y \cdot p_{j}^{\prime \prime} \in \mathbb{R}_{] 0,1]} \mid 1 \leq j \leq m\right\}
$$

If we take $x=\frac{p^{\prime \prime}-p}{p^{\prime \prime}-p^{\prime}}$ and $y=\frac{p-p^{\prime}}{p^{\prime \prime}-p^{\prime}}$, then $s_{2} \stackrel{a}{\longrightarrow}_{\mathrm{c}}\left(\frac{p^{\prime \prime}-p}{p^{\prime \prime}-p^{\prime}} \cdot \mathcal{D}_{2}^{\prime}+\frac{p-p^{\prime}}{p^{\prime \prime}-p^{\prime}} \cdot \mathcal{D}_{2}^{\prime \prime}\right)$ with:

$$
\begin{aligned}
\left(\frac{p^{\prime \prime}-p}{p^{\prime \prime}-p^{\prime}} \cdot \mathcal{D}_{2}^{\prime}+\frac{p-p^{\prime}}{p^{\prime \prime}-p^{\prime}} \cdot \mathcal{D}_{2}^{\prime \prime}\right)(\bigcup \mathcal{G}) & =\frac{p^{\prime \prime}-p}{p^{\prime \prime}-p^{\prime}} \cdot \mathcal{D}_{2}^{\prime}(\bigcup \mathcal{G})+\frac{p-p^{\prime}}{p^{\prime \prime}-p^{\prime}} \cdot \mathcal{D}_{2}^{\prime \prime}(\bigcup \mathcal{G}) \\
& =\frac{p^{\prime \prime}-p}{p^{\prime \prime}-p^{\prime}} \cdot p^{\prime}+\frac{p-p^{\prime}}{p^{\prime \prime}-p^{\prime}} \cdot p^{\prime \prime} \\
& =\frac{p^{\prime} \cdot p^{\prime \prime}-p \cdot p^{\prime}+p \cdot p^{\prime \prime}-p^{\prime} \cdot p^{\prime \prime}}{p^{\prime \prime}-p^{\prime}} \\
& =p \cdot \frac{p^{\prime \prime}-p^{\prime}}{p^{\prime \prime}-p^{\prime}} \\
& =p=\mathcal{D}_{1}(\bigcup \mathcal{G})
\end{aligned}
$$

Due to the generality of $\left(s_{1}, s_{2}\right) \in \mathcal{B}, a \in A$, and $\mathcal{G} \in 2^{S / \mathcal{B}}$, it turns out that $\mathcal{B}$ is also a $=$-group-by-group ct-probabilistic bisimulation, i.e., $s_{1} \sim_{\mathrm{PB}, \mathrm{gbg},=}^{\mathrm{ct}} s_{2}$. 


\section{Multistep Variants Inspired by $\sim_{B}$}

We start by introducing the multistep variant of $\sim_{\mathrm{B}}$ and proving that it coincides with $\sim_{\mathrm{B}}$ itself.

Definition 10. Let $(S, A, \longrightarrow)$ be an NPLTS in which the target of each transition is a Dirac distribution. A relation $\mathcal{B}$ over $S$ is a multistep bisimulation iff, whenever $\left(s_{1}, s_{2}\right) \in \mathcal{B}$, then for all traces $\alpha \in A^{*}$ it holds that:

- For each $s_{1} \stackrel{\alpha}{\Longrightarrow} \delta_{s_{1}^{\prime}}$ there exists $s_{2} \stackrel{\alpha}{\Longrightarrow} \delta_{s_{2}^{\prime}}$ such that $\left(s_{1}^{\prime}, s_{2}^{\prime}\right) \in \mathcal{B}$.

- For each $s_{2} \stackrel{\alpha}{\Longrightarrow} \delta_{s_{2}^{\prime}}$ there exists $s_{1} \stackrel{\alpha}{\Longrightarrow} \delta_{s_{1}^{\prime}}$ such that $\left(s_{1}^{\prime}, s_{2}^{\prime}\right) \in \mathcal{B}$.

We denote by $\sim_{\mathrm{B}, \mathrm{m}}$ the largest multistep bisimulation.

Theorem 12. Let $(S, A, \longrightarrow)$ be an NPLTS in which the target of each transition is a Dirac distribution. Let $s_{1}, s_{2} \in S$. Then:

$$
s_{1} \sim_{\mathrm{B}, \mathrm{m}} s_{2} \Longleftrightarrow s_{1} \sim_{\mathrm{B}} s_{2}
$$

Proof. Suppose that $s_{1} \sim_{\mathrm{B}, \mathrm{m}} s_{2}$. This means that there exists a multistep bisimulation $\mathcal{B}$ over $S$ such that $\left(s_{1}, s_{2}\right) \in \mathcal{B}$. As a consequence, it holds in particular that for all $\left(s_{1}^{\prime}, s_{2}^{\prime}\right) \in \mathcal{B}$ and $a \in A$ :

- For each $s_{1}^{\prime} \stackrel{a}{\Longrightarrow} \delta_{s_{1}^{\prime \prime}}$ there exists $s_{2}^{\prime} \stackrel{a}{\Longrightarrow} \delta_{s_{2}^{\prime \prime}}$ such that $\left(s_{1}^{\prime \prime}, s_{2}^{\prime \prime}\right) \in \mathcal{B}$.

- For each $s_{2}^{\prime} \stackrel{a}{\Longrightarrow} \delta_{s_{2}^{\prime \prime}}$ there exists $s_{1}^{\prime} \stackrel{a}{\Longrightarrow} \delta_{s_{1}^{\prime \prime}}$ such that $\left(s_{1}^{\prime \prime}, s_{2}^{\prime \prime}\right) \in \mathcal{B}$.

Since $\stackrel{a}{\Longrightarrow}$ coincides with $\stackrel{a}{\longrightarrow}$, we have that $\mathcal{B}$ is also a bisimulation and hence $s_{1} \sim_{\mathrm{B}} s_{2}$.

Suppose now that $s_{1} \sim_{\mathrm{B}} s_{2}$. This means that there exists a bisimulation $\mathcal{B}$ over $S$ such that $\left(s_{1}, s_{2}\right) \in \mathcal{B}$. We prove that $\mathcal{B}$ is also a multistep bisimulation, so that $s_{1} \sim_{\mathrm{B}, \mathrm{m}} s_{2}$ will follow. Given $s_{1}^{\prime}, s_{2}^{\prime} \in S$ such that $\left(s_{1}^{\prime}, s_{2}^{\prime}\right) \in \mathcal{B}$ and $\alpha \in A^{*}$, we proceed by induction on $|\alpha|$ :

- If $|\alpha|=0$, then $s_{1}^{\prime} \stackrel{\alpha}{\Longrightarrow} \delta_{s_{1}^{\prime}}$ and $s_{2}^{\prime} \stackrel{\alpha}{\Longrightarrow} \delta_{s_{2}^{\prime}}$ are the only possible computations from $s_{1}^{\prime}$ and $s_{2}^{\prime}$ labeled with $\alpha$, hence the result trivially holds.

- Let $|\alpha|=n \in \mathbb{N}_{>0}$ and suppose that the result holds for all traces of length $n-1$. Assume $\alpha=a \alpha^{\prime}$. Since $\left(s_{1}^{\prime}, s_{2}^{\prime}\right) \in \mathcal{B}$ and $\mathcal{B}$ is a bisimulation, it holds that for each $s_{1}^{\prime} \stackrel{a}{\longrightarrow} \delta_{s_{1}^{\prime \prime \prime}}$ there exists $s_{2}^{\prime} \stackrel{a}{\longrightarrow} \delta_{s_{2}^{\prime \prime \prime}}$ - and symmetrically for each $s_{2}^{\prime} \stackrel{a}{\longrightarrow} \delta_{s_{2}^{\prime \prime \prime}}$ there exists $s_{1}^{\prime} \stackrel{a}{\longrightarrow} \delta_{s_{1}^{\prime \prime \prime}}-$ such that $\left(s_{1}^{\prime \prime \prime}, s_{2}^{\prime \prime \prime}\right) \in \mathcal{B}$.

Suppose that $s_{1}^{\prime} \stackrel{\alpha}{\Longrightarrow} \delta_{s_{1}^{\prime \prime}}$ with $s_{1}^{\prime} \stackrel{a}{\longrightarrow} \delta_{s_{1}^{\prime \prime \prime}}$ and $s_{1}^{\prime \prime \prime} \stackrel{\alpha^{\prime}}{\longrightarrow} \delta_{s_{1}^{\prime \prime}}$. Then there exists $s_{2}^{\prime} \stackrel{a}{\longrightarrow} \delta_{s_{2}^{\prime \prime \prime}}$ such that $\left(s_{1}^{\prime \prime \prime}, s_{2}^{\prime \prime \prime}\right) \in \mathcal{B}$ and by the induction hypothesis there exists $s_{2}^{\prime \prime \prime} \stackrel{\alpha^{\prime}}{\Longrightarrow} \delta_{s_{2}^{\prime \prime}}$ such that $\left(s_{1}^{\prime \prime}, s_{2}^{\prime \prime}\right) \in \mathcal{B}$. As a consequence, there exists $s_{2}^{\prime} \stackrel{\alpha}{\Longrightarrow} \delta_{s_{2}^{\prime \prime}}$ such that $\left(s_{1}^{\prime \prime}, s_{2}^{\prime \prime}\right) \in \mathcal{B}$. Symmetrically, with a similar argument we derive that for each $s_{2}^{\prime} \stackrel{\alpha}{\Longrightarrow} \delta_{s_{2}^{\prime \prime}}$ there exists $s_{1}^{\prime} \stackrel{\alpha}{\Longrightarrow} \delta_{s_{1}^{\prime \prime}}$ such that $\left(s_{1}^{\prime \prime}, s_{2}^{\prime \prime}\right) \in \mathcal{B}$. 
We now provide the $\sim_{\mathrm{B}, \mathrm{m}}$-inspired definition of each of the probabilistic bisimilarities considered in this paper and prove that it coincides with the original one-step equivalence. The ct-variants of the $\sim_{\mathrm{B}, \mathrm{m}}$-inspired probabilistic bisimilarities can be defined similarly and satisfy an analogous coincidence property with respect to the original one-step ct-equivalences.

Definition 11. Let $(S, A, \longrightarrow)$ be an NPLTS. An equivalence relation $\mathcal{B}$ over $S$ is a multistep class-distribution probabilistic bisimulation iff, whenever $\left(s_{1}, s_{2}\right) \in \mathcal{B}$, then for all traces $\alpha \in A^{*}$ it holds that for each $s_{1} \stackrel{\alpha}{\Longrightarrow} \mathcal{D}_{1}$ there exists $s_{2} \stackrel{\alpha}{\Longrightarrow} \mathcal{D}_{2}$ such that, for all equivalence classes $C \in S / \mathcal{B}, \mathcal{D}_{1}(C)=\mathcal{D}_{2}(C)$. We denote by $\sim_{\mathrm{PB}, \mathrm{dis}, \mathrm{m}}$ the largest multistep class-distribution probabilistic bisimulation.

Theorem 13. Let $(S, A, \longrightarrow)$ be an NPLTS and $s_{1}, s_{2} \in S$. Then:

$$
s_{1} \sim_{\mathrm{PB}, \mathrm{dis}, \mathrm{m}} s_{2} \Longleftrightarrow s_{1} \sim_{\mathrm{PB}, \mathrm{dis}} s_{2}
$$

Proof. Suppose that $s_{1} \sim_{\mathrm{PB}, \mathrm{dis}, \mathrm{m}} s_{2}$. This means that there exists a multistep class-distribution probabilistic bisimulation $\mathcal{B}$ over $S$ such that $\left(s_{1}, s_{2}\right) \in \mathcal{B}$. As a consequence, it holds in particular that for all $\left(s_{1}^{\prime}, s_{2}^{\prime}\right) \in \mathcal{B}$ and $a \in A$, whenever $s_{1}^{\prime} \stackrel{a}{\Longrightarrow} \mathcal{D}_{1}$, then $s_{2}^{\prime} \stackrel{a}{\Longrightarrow} \mathcal{D}_{2}$ such that, for all $C \in S / \mathcal{B}, \mathcal{D}_{1}(C)=\mathcal{D}_{2}(C)$. Since $\stackrel{a}{\longrightarrow}$ coincides with $\stackrel{a}{\longrightarrow}$, we have that $\mathcal{B}$ is also a class-distribution probabilistic bisimulation and hence $s_{1} \sim_{\mathrm{PB}, \mathrm{dis}} s_{2}$.

Suppose now that $s_{1} \sim_{\mathrm{PB}, \mathrm{dis}} s_{2}$. This means that there exists a class-distribution probabilistic bisimulation $\mathcal{B}$ over $S$ such that $\left(s_{1}, s_{2}\right) \in \mathcal{B}$. We prove that $\mathcal{B}$ is also a multistep class-distribution probabilistic bisimulation, so that $s_{1} \sim \mathrm{PB}, \mathrm{dis}, \mathrm{m} s_{2}$ will follow. Given $s_{1}^{\prime}, s_{2}^{\prime} \in S$ such that $\left(s_{1}^{\prime}, s_{2}^{\prime}\right) \in \mathcal{B}$ and $\alpha \in A^{*}$, we proceed by induction on $|\alpha|$ :

- If $|\alpha|=0$, then $s_{1}^{\prime} \stackrel{\alpha}{\Longrightarrow} \delta_{s_{1}^{\prime}}$ and $s_{2}^{\prime} \stackrel{\alpha}{\Longrightarrow} \delta_{s_{2}^{\prime}}$ are the only possible computations from $s_{1}^{\prime}$ and $s_{2}^{\prime}$ labeled with $\alpha$ and for all $C \in S / \mathcal{B}$ it holds that:

$$
\delta_{s_{1}^{\prime}}(C)=\delta_{s_{2}^{\prime}}(C)= \begin{cases}1 & \text { if }\left\{s_{1}^{\prime}, s_{2}^{\prime}\right\} \subseteq C \\ 0 & \text { if }\left\{s_{1}^{\prime}, s_{2}^{\prime}\right\} \cap C=\emptyset\end{cases}
$$

because $\left(s_{1}^{\prime}, s_{2}^{\prime}\right) \in \mathcal{B}$ and $C$ is an equivalence class with respect to $\mathcal{B}$.

- Let $|\alpha|=n \in \mathbb{N}_{>0}$ and suppose that the result holds for all traces of length $n-1$. Assume $\alpha=a \alpha^{\prime}$. Since $\left(s_{1}^{\prime}, s_{2}^{\prime}\right) \in \mathcal{B}$ and $\mathcal{B}$ is a class-distribution probabilistic bisimulation, it holds that for each $s_{1}^{\prime} \stackrel{a}{\longrightarrow} \mathcal{D}_{1}^{\prime}$ there exists $s_{2}^{\prime} \stackrel{a}{\longrightarrow} \mathcal{D}_{2}^{\prime}$ such that, for all $C \in S / \mathcal{B}, \mathcal{D}_{1}^{\prime}(C)=\mathcal{D}_{2}^{\prime}(C)$.

Suppose that $s_{1}^{\prime} \stackrel{\alpha}{\Longrightarrow} \mathcal{D}_{1}$ with $s_{1}^{\prime} \stackrel{a}{\longrightarrow} \mathcal{D}_{1}^{\prime}, s_{1}^{\prime \prime} \stackrel{\alpha^{\prime}}{\longrightarrow} \mathcal{D}_{1}$, and $\mathcal{D}_{1}^{\prime}\left(s_{1}^{\prime \prime}\right)>0$. Then there exists $s_{2}^{\prime} \stackrel{a}{\longrightarrow} \mathcal{D}_{2}^{\prime}$ such that, for all $C \in S / \mathcal{B}, \mathcal{D}_{1}^{\prime}(C)=\mathcal{D}_{2}^{\prime}(C)$. If we take $s_{2}^{\prime \prime}$ such that $\left(s_{1}^{\prime \prime}, s_{2}^{\prime \prime}\right) \in \mathcal{B}$ and $\mathcal{D}_{2}^{\prime}\left(s_{2}^{\prime \prime}\right)>0$, by the induction hypothesis there exists $s_{2}^{\prime \prime} \stackrel{\alpha^{\prime}}{\Longrightarrow} \mathcal{D}_{2}$ such that, for all $C \in S / \mathcal{B}, \mathcal{D}_{1}(C)=\mathcal{D}_{2}(C)$. As a consequence, there exists $s_{2}^{\prime} \stackrel{\alpha}{\Longrightarrow} \mathcal{D}_{2}$ such that, for all $C \in S / \mathcal{B}, \mathcal{D}_{1}(C)=\mathcal{D}_{2}(C)$.

Definition 12. Let $(S, A, \longrightarrow)$ be an NPLTS and the relational operator $\bowtie \in$ $\{=, \leq, \geq\}$. An equivalence relation $\mathcal{B}$ over $S$ is a multistep $\bowtie$-group-by-group 
probabilistic bisimulation iff, whenever $\left(s_{1}, s_{2}\right) \in \mathcal{B}$, then for all traces $\alpha \in A^{*}$ and groups of equivalence classes $\mathcal{G} \in 2^{S / \mathcal{B}}$ it holds that for each $s_{1} \stackrel{\alpha}{\Longrightarrow} \mathcal{D}_{1}$ there exists $s_{2} \stackrel{\alpha}{\Longrightarrow} \mathcal{D}_{2}$ such that $\mathcal{D}_{1}(\bigcup \mathcal{G}) \bowtie \mathcal{D}_{2}(\bigcup \mathcal{G})$. We denote by $\sim_{\mathrm{PB}, \mathrm{gbg}, \bowtie, \mathrm{m}}$ the largest multistep $\bowtie$-group-by-group probabilistic bisimulation.

Theorem 14. Let $(S, A, \longrightarrow)$ be an NPLTS, $s_{1}, s_{2} \in S$, and $\bowtie \in\{=, \leq, \geq\}$. Then:

$$
s_{1} \sim_{\mathrm{PB}, \mathrm{gbg}, \bowtie, \mathrm{m}} s_{2} \Longleftrightarrow s_{1} \sim_{\mathrm{PB}, \mathrm{gbg}, \bowtie} s_{2}
$$

Proof. Suppose that $s_{1} \sim_{\mathrm{PB}, \mathrm{gbg}, \bowtie, \mathrm{m}} s_{2}$. This means that there exists a multistep $\bowtie$-group-by-group probabilistic bisimulation $\mathcal{B}$ over $S$ such that $\left(s_{1}, s_{2}\right) \in \mathcal{B}$. As a consequence, it holds in particular that for all $\left(s_{1}^{\prime}, s_{2}^{\prime}\right) \in \mathcal{B}, a \in A$, and $\mathcal{G} \in$ $2^{S / \mathcal{B}}$, whenever $s_{1}^{\prime} \stackrel{a}{\Longrightarrow} \mathcal{D}_{1}$, then $s_{2}^{\prime} \stackrel{a}{\Longrightarrow} \mathcal{D}_{2}$ such that $\mathcal{D}_{1}(\bigcup \mathcal{G}) \bowtie \mathcal{D}_{2}(\bigcup \mathcal{G})$. Since $\stackrel{a}{\longrightarrow}$ coincides with $\stackrel{a}{\longrightarrow}$, we have that $\mathcal{B}$ is also $a \bowtie$-group-by-group probabilistic bisimulation and hence $s_{1} \sim_{\mathrm{PB}, \mathrm{gbg}, \bowtie} s_{2}$.

Suppose now that $s_{1} \sim \sim_{\mathrm{PB}, \mathrm{gbg}, \bowtie} s_{2}$. This means that there exists a $\bowtie$-groupby-group probabilistic bisimulation $\mathcal{B}$ over $S$ such that $\left(s_{1}, s_{2}\right) \in \mathcal{B}$. We prove that $\mathcal{B}$ is also a multistep $\bowtie$-group-by-group probabilistic bisimulation, so that $s_{1} \sim_{\mathrm{PB}, \mathrm{gbg}, \bowtie, \mathrm{m}} s_{2}$ will follow. Given $s_{1}^{\prime}, s_{2}^{\prime} \in S$ such that $\left(s_{1}^{\prime}, s_{2}^{\prime}\right) \in \mathcal{B}, \alpha \in A^{*}$, and $\mathcal{G} \in 2^{S / \mathcal{B}}$, we proceed by induction on $|\alpha|$ :

- If $|\alpha|=0$, then $s_{1}^{\prime} \stackrel{\alpha}{\Longrightarrow} \delta_{s_{1}^{\prime}}$ and $s_{2}^{\prime} \stackrel{\alpha}{\Longrightarrow} \delta_{s_{2}^{\prime}}$ are the only possible computations from $s_{1}^{\prime}$ and $s_{2}^{\prime}$ labeled with $\alpha$ and it holds that:

$$
\delta_{s_{1}^{\prime}}(\cup \mathcal{G})=\delta_{s_{2}^{\prime}}(\cup \mathcal{G})= \begin{cases}1 & \text { if }\left\{s_{1}^{\prime}, s_{2}^{\prime}\right\} \subseteq C \text { for some } C \in \mathcal{G} \\ 0 & \text { if }\left\{s_{1}^{\prime}, s_{2}^{\prime}\right\} \cap C=\emptyset \text { for all } C \in \mathcal{G}\end{cases}
$$

because $\left(s_{1}^{\prime}, s_{2}^{\prime}\right) \in \mathcal{B}$ and $\mathcal{G}$ is a group of equivalence classes with respect to $\mathcal{B}$.

- Let $|\alpha|=n \in \mathbb{N}_{>0}$ and suppose that the result holds for all traces of length $n-1$. Assume $\alpha=a \alpha^{\prime}$. Since $\left(s_{1}^{\prime}, s_{2}^{\prime}\right) \in \mathcal{B}$ and $\mathcal{B}$ is a $\bowtie$-group-by-group probabilistic bisimulation, for all $\mathcal{G}^{\prime} \in 2^{S / \mathcal{B}}$ it holds that for each $s_{1}^{\prime} \stackrel{a}{\longrightarrow} \mathcal{D}_{1}^{\prime}$ there exists $s_{2}^{\prime} \stackrel{a}{\longrightarrow} \mathcal{D}_{2}^{\prime}$ such that $\mathcal{D}_{1}^{\prime}\left(\bigcup \mathcal{G}^{\prime}\right) \bowtie \mathcal{D}_{2}^{\prime}\left(\bigcup \mathcal{G}^{\prime}\right)$.

Suppose that $s_{1}^{\prime} \stackrel{\alpha}{\Longrightarrow} \mathcal{D}_{1}$ with $s_{1}^{\prime} \stackrel{a}{\longrightarrow} \mathcal{D}_{1}^{\prime}, s_{1}^{\prime \prime} \stackrel{\alpha^{\prime}}{\Longrightarrow} \mathcal{D}_{1}$, and $\mathcal{D}_{1}^{\prime}\left(s_{1}^{\prime \prime}\right)>0$. Let $\mathcal{G}^{\prime}=$ $\left\{C^{\prime}\right\}$ with $C^{\prime}$ being the equivalence class containing $s_{1}^{\prime \prime}$. Then there exists $s_{2}^{\prime} \stackrel{a}{\longrightarrow} \mathcal{D}_{2}^{\prime}$ such that $\mathcal{D}_{1}^{\prime}\left(\bigcup \mathcal{G}^{\prime}\right) \bowtie \mathcal{D}_{2}^{\prime}\left(\bigcup \mathcal{G}^{\prime}\right)$. If we take $s_{2}^{\prime \prime}$ such that $\left(s_{1}^{\prime \prime}, s_{2}^{\prime \prime}\right) \in$ $\mathcal{B}$ and $\mathcal{D}_{2}^{\prime}\left(s_{2}^{\prime \prime}\right)>0$ - it obviously exists in the case that $\bowtie \in\{=, \leq\}$ because $\mathcal{D}_{1}^{\prime}\left(s_{1}^{\prime \prime}\right)>0$, and it also exists in the case that $\bowtie$ is $\geq$ because, if $s_{2}^{\prime}$ had no a-transition reaching $\mathcal{G}^{\prime}$ with probability greater than 0 , then all a-transitions of $s_{2}^{\prime}$ would reach $\mathcal{G}^{\prime \prime}=2^{S / \mathcal{B}} \backslash \mathcal{G}^{\prime}$ with probability 1 and hence for the transition $s_{1}^{\prime} \stackrel{a}{\longrightarrow} \mathcal{D}_{1}^{\prime}$ we would have $\mathcal{D}_{1}^{\prime}\left(\bigcup \mathcal{G}^{\prime \prime}\right)=1-\mathcal{D}_{1}^{\prime}\left(\bigcup \mathcal{G}^{\prime}\right)<1=\mathcal{D}_{2}^{\prime}\left(\bigcup \mathcal{G}^{\prime \prime}\right)$ for all transitions $s_{2}^{\prime} \stackrel{a}{\longrightarrow} \mathcal{D}_{2}^{\prime}$, i.e., $\mathcal{B}$ would not be a $\geq$-group-by-group probabilistic bisimulation - by the induction hypothesis there exists $s_{2}^{\prime \prime} \stackrel{\alpha^{\prime}}{\Longrightarrow} \mathcal{D}_{2}$ such that $\mathcal{D}_{1}(\bigcup \mathcal{G}) \bowtie \mathcal{D}_{2}(\bigcup \mathcal{G})$. As a consequence, there exists $s_{2}^{\prime} \stackrel{\alpha}{\Longrightarrow} \mathcal{D}_{2}$ such that $\mathcal{D}_{1}(\bigcup \mathcal{G}) \bowtie \mathcal{D}_{2}(\bigcup \mathcal{G})$.

Definition 13. Let $(S, A, \longrightarrow)$ be an NPLTS. An equivalence relation $\mathcal{B}$ over $S$ is a multistep $\sqcup П$-group-by-group probabilistic bisimulation iff, whenever $\left(s_{1}, s_{2}\right)$ 
$\in \mathcal{B}$, then for all traces $\alpha \in A^{*}$ and groups of equivalence classes $\mathcal{G} \in 2^{S / \mathcal{B}}$ it holds that $s_{1} \stackrel{\alpha}{\Longrightarrow}$ implies $s_{2} \stackrel{\alpha}{\Longrightarrow}$ with:

$$
\begin{aligned}
& \stackrel{\bigsqcup_{s_{1}} \stackrel{\alpha}{\Longrightarrow} \mathcal{D}_{1}}{\mathcal{D}_{1}(\bigcup \mathcal{G})=} \bigsqcup_{s_{2} \stackrel{\alpha}{\Longrightarrow} \mathcal{D}_{2}} \mathcal{D}_{2}(\bigcup \mathcal{G}) \\
& \prod_{s_{1} \stackrel{\alpha}{\Longrightarrow} \mathcal{D}_{1}} \mathcal{D}_{1}(\bigcup \mathcal{G})=\prod_{s_{2} \stackrel{\alpha}{\Longrightarrow} \mathcal{D}_{2}} \mathcal{D}_{2}(\bigcup \mathcal{G})
\end{aligned}
$$

We denote by $\sim_{\mathrm{PB}, \mathrm{gbg}, \sqcup \sqcap, \mathrm{m}}$ the largest multistep $\sqcup \sqcap$-group-by-group probabilistic bisimulation.

Theorem 15. Let $(S, A, \longrightarrow)$ be an NPLTS and $s_{1}, s_{2} \in S$. Then:

$$
s_{1} \sim_{\mathrm{PB}, \mathrm{gbg}, \sqcup \sqcap, \mathrm{m}} s_{2} \Longleftrightarrow s_{1} \sim_{\mathrm{PB}, \mathrm{gbg}, \sqcup \sqcap} s_{2}
$$

Proof. Suppose that $s_{1} \sim_{\mathrm{PB}, \mathrm{gbg}, \sqcup \sqcap, \mathrm{m}} s_{2}$. This means that there exists a multistep $\sqcup \sqcap$-group-by-group probabilistic bisimulation $\mathcal{B}$ over $S$ such that $\left(s_{1}, s_{2}\right) \in \mathcal{B}$. As a consequence, it holds in particular that for all $\left(s_{1}^{\prime}, s_{2}^{\prime}\right) \in \mathcal{B}, a \in A$, and $\mathcal{G} \in 2^{S / \mathcal{B}}$, whenever $s_{1}^{\prime} \stackrel{a}{\Longrightarrow}$, then $s_{2}^{\prime} \stackrel{a}{\Longrightarrow}$ with:

$$
\begin{aligned}
& \stackrel{\bigsqcup}{s_{1}^{\prime} \stackrel{a}{\Rightarrow} \mathcal{D}_{1}} \mathcal{D}_{1}(\bigcup \mathcal{G})=\bigsqcup_{s_{2}^{\prime} \stackrel{\bigsqcup^{\prime}}{\Longrightarrow} \mathcal{D}_{2}} \mathcal{D}_{2}(\bigcup \mathcal{G}) \\
& \prod_{s_{1}^{\prime} \stackrel{a}{\Longrightarrow} \mathcal{D}_{1}} \mathcal{D}_{1}(\bigcup \mathcal{G})=\prod_{s_{2}^{\prime} \stackrel{a}{\Longrightarrow} \mathcal{D}_{2}} \mathcal{D}_{2}(\bigcup \mathcal{G})
\end{aligned}
$$

Since $\stackrel{a}{\Longrightarrow}$ coincides with $\stackrel{a}{\longrightarrow}$, we have that $\mathcal{B}$ is also $a \sqcup \sqcap$-group-by-group probabilistic bisimulation and hence $s_{1} \sim_{\mathrm{PB}, \mathrm{gbg}, \sqcup \sqcap} s_{2}$.

Suppose now that $s_{1} \sim_{\mathrm{PB}, \mathrm{gbg}, \sqcup \sqcap} s_{2}$. This means that there exists a பП-groupby-group probabilistic bisimulation $\mathcal{B}$ over $S$ such that $\left(s_{1}, s_{2}\right) \in \mathcal{B}$. We prove that $\mathcal{B}$ is also a multistep $\sqcup П$-group-by-group probabilistic bisimulation, so that $s_{1} \sim_{\mathrm{PB}, \mathrm{gbg}, \sqcup \sqcap, \mathrm{m}} s_{2}$ will follow. Given $s_{1}^{\prime}, s_{2}^{\prime} \in S$ such that $\left(s_{1}^{\prime}, s_{2}^{\prime}\right) \in \mathcal{B}, \alpha \in A^{*}$, and $\mathcal{G} \in 2^{S / \mathcal{B}}$, we proceed by induction on $|\alpha|$ :

- If $|\alpha|=0$, then $s_{1}^{\prime} \stackrel{\alpha}{\Longrightarrow} \delta_{s_{1}^{\prime}}$ and $s_{2}^{\prime} \stackrel{\alpha}{\Longrightarrow} \delta_{s_{2}^{\prime}}$ are the only possible computations from $s_{1}^{\prime}$ and $s_{2}^{\prime}$ labeled with $\alpha$ and it holds that:

$$
\delta_{s_{1}^{\prime}}(\cup \mathcal{G})=\delta_{s_{2}^{\prime}}(\bigcup \mathcal{G})= \begin{cases}1 & \text { if }\left\{s_{1}^{\prime}, s_{2}^{\prime}\right\} \subseteq C \text { for some } C \in \mathcal{G} \\ 0 & \text { if }\left\{s_{1}^{\prime}, s_{2}^{\prime}\right\} \cap C=\emptyset \text { for all } C \in \mathcal{G}\end{cases}
$$

because $\left(s_{1}^{\prime}, s_{2}^{\prime}\right) \in \mathcal{B}$ and $\mathcal{G}$ is a group of equivalence classes with respect to $\mathcal{B}$. Therefore:

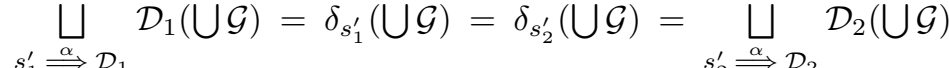

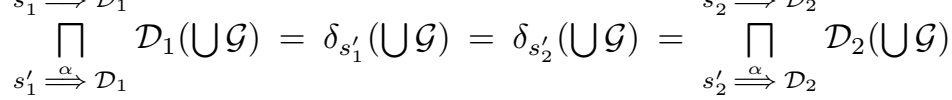

- Let $|\alpha|=n \in \mathbb{N}_{>0}$ and suppose that the result holds for all traces of length $n-1$. Assume $\alpha=a \alpha^{\prime}$. Since $\left(s_{1}^{\prime}, s_{2}^{\prime}\right) \in \mathcal{B}$ and $\mathcal{B}$ is a பП-group-by-group probabilistic bisimulation, for all $\mathcal{G}^{\prime} \in 2^{S / \mathcal{B}}$ it holds that $s_{1}^{\prime} \stackrel{a}{\longrightarrow}$ implies $s_{2}^{\prime} \stackrel{a}{\longrightarrow}$ with:

$$
\begin{aligned}
& \bigsqcup_{s_{1}^{\prime} \stackrel{a}{\longrightarrow} \mathcal{D}_{1}^{\prime}} \mathcal{D}_{1}^{\prime}\left(\bigcup \mathcal{G}^{\prime}\right)=\bigsqcup_{s_{2}^{\prime}}^{\bigsqcup_{a}^{\longrightarrow} \mathcal{D}_{2}^{\prime}} \mathcal{D}_{2}^{\prime}\left(\bigcup \mathcal{G}^{\prime}\right) \\
& \prod_{s_{1}^{\prime} \stackrel{a}{\longrightarrow} \mathcal{D}_{1}^{\prime}} \mathcal{D}_{1}^{\prime}\left(\cup \mathcal{G}^{\prime}\right)=\prod_{s_{2}^{\prime} \stackrel{a}{\longrightarrow} \mathcal{D}_{2}^{\prime}} \mathcal{D}_{2}^{\prime}\left(\bigcup \mathcal{G}^{\prime}\right)
\end{aligned}
$$

Suppose that $s_{1}^{\prime} \stackrel{\alpha}{\Longrightarrow}$ with $s_{1}^{\prime} \stackrel{a}{\longrightarrow} \mathcal{D}_{1}^{\prime}, s_{1}^{\prime \prime} \stackrel{\alpha^{\prime}}{\Longrightarrow}$, and $\mathcal{D}_{1}^{\prime}\left(s_{1}^{\prime \prime}\right)>0$. Let $\mathcal{G}^{\prime}=\left\{C^{\prime}\right\}$ 
with $C^{\prime}$ being the equivalence class containing $s_{1}^{\prime \prime}$. Then $s_{2}^{\prime} \stackrel{a}{\longrightarrow}$ with:

$$
\begin{aligned}
& \underset{s_{1}^{\prime} \stackrel{\bigsqcup_{a}}{\longrightarrow} \mathcal{D}_{1}^{\prime}}{ } \mathcal{D}_{1}^{\prime}\left(\bigcup \mathcal{G}^{\prime}\right)=\bigsqcup_{s_{2}^{\prime} \stackrel{a}{\longrightarrow} \mathcal{D}_{2}^{\prime}} \mathcal{D}_{2}^{\prime}\left(\bigcup \mathcal{G}^{\prime}\right) \\
& \prod_{s_{1}^{\prime} \stackrel{a}{\longrightarrow} \mathcal{D}_{1}^{\prime}} \mathcal{D}_{1}^{\prime}\left(\bigcup \mathcal{G}^{\prime}\right)=\prod_{s_{2}^{\prime} \stackrel{a}{\longrightarrow} \mathcal{D}_{2}^{\prime}} \mathcal{D}_{2}^{\prime}\left(\bigcup \mathcal{G}^{\prime}\right)
\end{aligned}
$$

If we take $s_{2}^{\prime \prime}$ and $\mathcal{D}_{2}^{\prime}$ such that $\left(s_{1}^{\prime \prime}, s_{2}^{\prime \prime}\right) \in \mathcal{B}, \mathcal{D}_{2}^{\prime}\left(s_{2}^{\prime \prime}\right)>0$, and $s_{2}^{\prime} \stackrel{a}{\longrightarrow} \mathcal{D}_{2}^{\prime}$, by the induction hypothesis we have that $s_{2}^{\prime \prime} \stackrel{\alpha^{\prime}}{\Longrightarrow}$ with:

$$
\begin{aligned}
& \bigsqcup \mathcal{D}_{1}(\bigcup \mathcal{G})=\quad \bigsqcup \mathcal{D}_{2}(\bigcup \mathcal{G}) \\
& s_{1}^{\prime \prime} \stackrel{\alpha^{\prime}}{\Rightarrow} \mathcal{D}_{1} \quad s_{2}^{\prime \prime} \stackrel{\alpha^{\prime}}{\Rightarrow} \mathcal{D}_{2} \\
& \prod \mathcal{D}_{1}(\bigcup \mathcal{G})=\prod \mathcal{D}_{2}(\bigcup \mathcal{G}) \\
& s_{1}^{\prime \prime} \stackrel{\alpha^{\prime}}{\Longrightarrow} \mathcal{D}_{1} \quad \quad s_{2}^{\prime \prime} \stackrel{\alpha^{\prime}}{\Longrightarrow} \mathcal{D}_{2}
\end{aligned}
$$

As a consequence, $s_{2}^{\prime} \stackrel{\alpha}{\Longrightarrow}$ with:

$$
\begin{aligned}
& \bigsqcup_{s_{1}^{\prime} \stackrel{\alpha^{\prime}}{\Longrightarrow} \mathcal{D}_{1}} \mathcal{D}_{1}(\bigcup \mathcal{G})=\bigsqcup_{s_{2}^{\prime} \stackrel{\bigsqcup_{\alpha}}{\Longrightarrow} \mathcal{D}_{2}} \mathcal{D}_{2}(\bigcup \mathcal{G}) \\
& \prod \mathcal{D}_{1}(\cup \mathcal{G})=\prod \mathcal{D}_{2}(\cup \mathcal{G})
\end{aligned}
$$

Definition 14. Let $(S, A, \longrightarrow)$ be an NPLTS and symbol $\# \in\{\bigsqcup, \Pi\}$. An equivalence relation $\mathcal{B}$ over $S$ is a multistep \#-group-by-group probabilistic bisimulation iff, whenever $\left(s_{1}, s_{2}\right) \in \mathcal{B}$, then for all traces $\alpha \in A^{*}$ and groups of equivalence classes $\mathcal{G} \in 2^{S / \mathcal{B}}$ it holds that $s_{1} \stackrel{\alpha}{\Longrightarrow}$ implies $s_{2} \stackrel{\alpha}{\Longrightarrow}$ with:

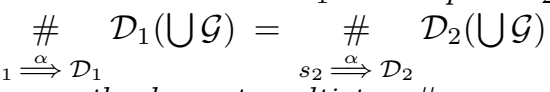

We denote by $\sim_{\mathrm{PB}, \mathrm{gbg}, \#, \mathrm{~m}}$ the largest multistep \#-group-by-group probabilistic bisimulation.

Theorem 16. Let $(S, A, \longrightarrow)$ be an NPLTS, $s_{1}, s_{2} \in S$, and $\# \in\{\bigsqcup, \sqcap\}$. Then:

$$
s_{1} \sim_{\mathrm{PB}, \mathrm{gbg}, \#, \mathrm{~m}} s_{2} \Longleftrightarrow s_{1} \sim_{\mathrm{PB}, \mathrm{gbg}, \#} s_{2}
$$

Proof. Similar to the proof of Thm. 15. With regard to the induction step of the proof that $s_{1} \sim_{\mathrm{PB}, \mathrm{gbg}, \#} s_{2}$ implies $s_{1} \sim_{\mathrm{PB}, \mathrm{gbg}, \#, \mathrm{~m}} s_{2}$, we observe that $s_{2}^{\prime \prime}$ and $\mathcal{D}_{2}^{\prime}$ such that $\left(s_{1}^{\prime \prime}, s_{2}^{\prime \prime}\right) \in \mathcal{B}, \mathcal{D}_{2}^{\prime}\left(s_{2}^{\prime \prime}\right)>0$, and $s_{2}^{\prime} \stackrel{a}{\longrightarrow} \mathcal{D}_{2}^{\prime}$ obviously exist in the case that \# is $\sqcup$ because $\mathcal{D}_{1}^{\prime}\left(s_{1}^{\prime \prime}\right)>0$. They also exist in the case that \# is $\square$ because, if $s_{2}^{\prime}$ had no a-transition reaching $\mathcal{G}^{\prime}$ (the group composed only of the equivalence class containing $\left.s_{1}^{\prime \prime}\right)$ with probability greater than 0 , then all a-transitions of $s_{2}^{\prime}$ would reach $\mathcal{G}^{\prime \prime}=2^{S / \mathcal{B}} \backslash \mathcal{G}^{\prime}$ with probability 1 and hence we would have:

$$
\prod_{s_{1}^{\prime} \stackrel{a}{\longrightarrow} \mathcal{D}_{1}^{\prime}} \mathcal{D}_{1}^{\prime}\left(\bigcup \mathcal{G}^{\prime \prime}\right)<1=\prod_{s_{2}^{\prime} \stackrel{a}{\longrightarrow} \mathcal{D}_{2}^{\prime}} \mathcal{D}_{2}^{\prime}\left(\bigcup \mathcal{G}^{\prime \prime}\right)
$$

i.e., the considered relation $\mathcal{B}$ would not be a $\sqcap$-group-by-group probabilistic bisimulation

We conclude by showing that all the considered $\sim_{\mathrm{B}, \mathrm{m}}$-inspired probabilistic bisimilarities collapse into $\sim_{\mathrm{B}, \mathrm{m}}$ when restricting attention to fully nondeterministic processes. An analogous result holds for their ct-variants.

Theorem 17. Let $(S, A, \longrightarrow)$ be an NPLTS in which the target of each transition is a Dirac distribution. Let $s_{1}, s_{2} \in S$ and $\circ \in\{=, \leq, \geq, \sqcup \sqcap, \sqcup, \sqcap\}$. Then: 


$$
s_{1} \sim_{\mathrm{PB}, \mathrm{dis}, \mathrm{m}} s_{2} \Longleftrightarrow s_{1} \sim_{\mathrm{PB}, \mathrm{gbg}, \mathrm{o}, \mathrm{m}} s_{2} \Longleftrightarrow s_{1} \sim_{\mathrm{B}, \mathrm{m}} s_{2}
$$

Proof. Since every multistep transition of this specific NPLTS can reach with probability greater than 0 a single state and hence a single class of any equivalence relation - which are thus reached with probability 1 - the reflexive, symmetric, and transitive closure of a multistep bisimulation is trivially a multistep classdistribution probabilistic bisimulation, a multistep =-group-by-group probabilistic bisimulation, a multistep $\leq$-group-by-group probabilistic bisimulation, a multistep $\geq$-group-by-group probabilistic bisimulation, a multistep $\sqcup П$-group-by-group probabilistic bisimulation, a multistep $\sqcup$-group-by-group probabilistic bisimulation, and a multistep $\sqcap$-group-by-group probabilistic bisimulation. 


\section{Multistep Variants Inspired by $\sim_{\mathrm{PB}}$}

We start by introducing the multistep variant of $\sim_{\mathrm{PB}}$ and proving that it coincides with $\sim_{\mathrm{PB}}$ itself. Given an $\operatorname{NPLTS}(S, A, \longrightarrow)$ in which the transitions of each state have different labels and given $s \in S, \alpha \in A^{*}$, and $S^{\prime} \subseteq S$, we inductively define the multistep probability of reaching a state in $S^{\prime}$ from $s$ via $\alpha$ as follows:

$$
\operatorname{prob}_{\mathrm{m}}\left(s, \alpha, S^{\prime}\right)= \begin{cases}\sum_{s^{\prime} \in S} \mathcal{D}\left(s^{\prime}\right) \cdot \operatorname{prob}_{\mathrm{m}}\left(s^{\prime}, \alpha^{\prime}, S^{\prime}\right) & \text { if } \alpha=a \alpha^{\prime} \text { and } s \stackrel{a}{\longrightarrow} \mathcal{D} \\ 1 & \text { if } \alpha=\varepsilon \text { and } s \in S^{\prime} \\ 0 & \text { if } \alpha=a \alpha^{\prime} \text { and } s \stackrel{a}{\longrightarrow} \\ & \text { or } \alpha=\varepsilon \text { and } s \notin S^{\prime}\end{cases}
$$

Definition 15. Let $(S, A, \longrightarrow)$ be an NPLTS in which the transitions of each state have different labels. An equivalence relation $\mathcal{B}$ over $S$ is a p-multistep probabilistic bisimulation iff, whenever $\left(s_{1}, s_{2}\right) \in \mathcal{B}$, then for all traces $\alpha \in A^{*}$ and equivalence classes $C \in S / \mathcal{B}$ it holds that $s_{1} \stackrel{\alpha}{\Longrightarrow}$ implies $s_{2} \stackrel{\alpha}{\Longrightarrow}$ with:

$$
\operatorname{prob}_{\mathrm{m}}\left(s_{1}, \alpha, C\right)=\operatorname{prob}_{\mathrm{m}}\left(s_{2}, \alpha, C\right)
$$

We denote by $\sim_{\mathrm{PB}, \mathrm{pm}}$ the largest p-multistep probabilistic bisimulation.

Theorem 18. Let $(S, A, \longrightarrow)$ be an NPLTS in which the transitions of each state have different labels. Let $s_{1}, s_{2} \in S$. Then:

$$
s_{1} \sim_{\mathrm{PB}, \mathrm{pm}} s_{2} \Longleftrightarrow s_{1} \sim_{\mathrm{PB}} s_{2}
$$

Proof. Suppose that $s_{1} \sim_{\mathrm{PB}, \mathrm{pm}} s_{2}$. This means that there exists a p-multistep probabilistic bisimulation $\mathcal{B}$ over $S$ such that $\left(s_{1}, s_{2}\right) \in \mathcal{B}$. As a consequence, it holds in particular that for all $\left(s_{1}^{\prime}, s_{2}^{\prime}\right) \in \mathcal{B}, a \in A$, and $C \in S / \mathcal{B}$, whenever $s_{1}^{\prime} \stackrel{a}{\Longrightarrow}$, then $s_{2}^{\prime} \stackrel{a}{\Longrightarrow}$ with:

$$
\operatorname{prob}_{\mathrm{m}}\left(s_{1}^{\prime}, a, C\right)=\operatorname{prob}_{\mathrm{m}}\left(s_{2}^{\prime}, a, C\right)
$$

Since $\stackrel{a}{\longrightarrow}$ coincides with $\stackrel{a}{\longrightarrow}$ and for all $s \in S$ such that $s \stackrel{a}{\longrightarrow} \mathcal{D}$ it holds that:

$$
\operatorname{prob}_{\mathrm{m}}(s, a, C)=\sum_{s^{\prime} \in C} \mathcal{D}\left(s^{\prime}\right)=\mathcal{D}(C)
$$

we have that $s_{1}^{\prime} \stackrel{a}{\longrightarrow} \mathcal{D}_{1}$ implies $s_{2}^{\prime} \stackrel{a}{\longrightarrow} \mathcal{D}_{2}$ with $\mathcal{D}_{1}(C)=\mathcal{D}_{2}(C)$. In other words, $\mathcal{B}$ is also a probabilistic bisimulation and hence $s_{1} \sim_{\mathrm{PB}} s_{2}$.

Suppose now that $s_{1} \sim_{\mathrm{PB}} s_{2}$. This means that there exists a probabilistic bisimulation $\mathcal{B}$ over $S$ such that $\left(s_{1}, s_{2}\right) \in \mathcal{B}$. We prove that $\mathcal{B}$ is also a p-multistep probabilistic bisimulation, so that $s_{1} \sim \mathrm{PB}, \mathrm{pm} s_{2}$ will follow. Given $s_{1}^{\prime}, s_{2}^{\prime} \in S$ such that $\left(s_{1}^{\prime}, s_{2}^{\prime}\right) \in \mathcal{B}, \alpha \in A^{*}$, and $C \in S / \mathcal{B}$, we proceed by induction on $|\alpha|$ :

- If $|\alpha|=0$, then $s_{1}^{\prime} \stackrel{\alpha}{\Longrightarrow} \delta_{s_{1}^{\prime}}$ and $s_{2}^{\prime} \stackrel{\alpha}{\Longrightarrow} \delta_{s_{2}^{\prime}}$ are the only possible computations from $s_{1}^{\prime}$ and $s_{2}^{\prime}$ labeled with $\alpha$ and it holds that:

$$
\operatorname{prob}_{\mathrm{m}}\left(s_{1}^{\prime}, \alpha, C\right)=\operatorname{prob}_{\mathrm{m}}\left(s_{2}^{\prime}, \alpha, C\right)= \begin{cases}1 & \text { if }\left\{s_{1}^{\prime}, s_{2}^{\prime}\right\} \subseteq C \\ 0 & \text { if }\left\{s_{1}^{\prime}, s_{2}^{\prime}\right\} \cap C=\emptyset\end{cases}
$$

because $\left(s_{1}^{\prime}, s_{2}^{\prime}\right) \in \mathcal{B}$ and $C$ is an equivalence class with respect to $\mathcal{B}$.

- Let $|\alpha|=n \in \mathbb{N}_{>0}$ and suppose that the result holds for all traces of length $n-1$. Assume $\alpha=a \alpha^{\prime}$. Since $\left(s_{1}^{\prime}, s_{2}^{\prime}\right) \in \mathcal{B}$ and $\mathcal{B}$ is a probabilistic bisimulation, for all $C^{\prime} \in S / \mathcal{B}$ it holds that for each $s_{1}^{\prime} \stackrel{a}{\longrightarrow} \mathcal{D}_{1}$ there exists $s_{2}^{\prime} \stackrel{a}{\longrightarrow} \mathcal{D}_{2}$ 
such that $\mathcal{D}_{1}\left(C^{\prime}\right)=\mathcal{D}_{2}\left(C^{\prime}\right)$.

$$
\begin{aligned}
& \text { Given } s \in S \text { such that } s \stackrel{\alpha}{\Longrightarrow} \text { with } s \stackrel{a}{\longrightarrow} \mathcal{D} \text {, it holds that: } \\
& \operatorname{prob}_{\mathrm{m}}(s, \alpha, C)=\sum_{s^{\prime} \in S} \mathcal{D}\left(s^{\prime}\right) \cdot \text { prob }_{\mathrm{m}}\left(s^{\prime}, \alpha^{\prime}, C\right) \\
&=\sum_{C^{\prime} \in S / \mathcal{B}} \sum_{s^{\prime} \in C^{\prime}} \mathcal{D}\left(s^{\prime}\right) \cdot \operatorname{prob}_{\mathrm{m}}\left(s^{\prime}, \alpha^{\prime}, C\right) \\
&=\sum_{C^{\prime} \in S / \mathcal{B}} \sum_{s^{\prime} \in C^{\prime}} \mathcal{D}\left(s^{\prime}\right) \cdot \operatorname{prob}_{\mathrm{m}}\left(s_{C^{\prime}}, \alpha^{\prime}, C\right) \\
&=\sum_{C^{\prime} \in S / \mathcal{B}} \operatorname{prob}_{\mathrm{m}}\left(s_{C^{\prime}}, \alpha^{\prime}, C\right) \cdot \sum_{s^{\prime} \in C^{\prime}} \mathcal{D}\left(s^{\prime}\right) \\
&=\sum_{C^{\prime} \in S / \mathcal{B}} \operatorname{prob}_{\mathrm{m}}\left(s_{C^{\prime}}, \alpha^{\prime}, C\right) \cdot \mathcal{D}\left(C^{\prime}\right)
\end{aligned}
$$

where $s_{C^{\prime}} \in C^{\prime}$ and the factorization of $\operatorname{prob}_{\mathrm{m}}\left(s_{C^{\prime}}, \alpha^{\prime}, C\right)$ stems from the application of the induction hypothesis on $\alpha^{\prime}$ to all states of each equivalence class $C^{\prime}$. Since for each $s_{1}^{\prime} \stackrel{a}{\longrightarrow} \mathcal{D}_{1}$ there exists $s_{2}^{\prime} \stackrel{a}{\longrightarrow} \mathcal{D}_{2}$ such that $\mathcal{D}_{1}\left(C^{\prime}\right)=\mathcal{D}_{2}\left(C^{\prime}\right)$ for all $C^{\prime} \in S / \mathcal{B}$ - remember that the quantification over $C^{\prime}$ can be equivalently anticipated or postponed in the absence of internal nondeterminism - we derive that, whenever $s_{1}^{\prime} \stackrel{\alpha}{\Longrightarrow}$, then $s_{2}^{\prime} \stackrel{\alpha}{\Longrightarrow}$ with:

$$
\operatorname{prob}_{\mathrm{m}}\left(s_{1}^{\prime}, \alpha, C\right)=\operatorname{prob}_{\mathrm{m}}\left(s_{2}^{\prime}, \alpha, C\right)
$$

When considering an arbitrary NPLTS $(S, A, \longrightarrow)$, internal nondeterminism comes into play and hence there might be several computations labeled with the same trace belonging to different resolutions of nondeterminism. In that case, their multistep probabilities have to be kept separate, otherwise their multistep probabilities can be summed up like in the case of reactive probabilistic processes.

Since preserving the connection between each computation and the resolution of nondeterminism to which it belongs is important to define a $\sim_{\mathrm{PB}, \mathrm{m}}$-inspired multistep variant of $\sim_{\mathrm{PB}, \mathrm{dis}}$, we formalize below the notion of resolution. We call resolution of a state $s$ of an NPLTS $\mathcal{U}$ any possible way of resolving nondeterminism starting from $s$. Each resolution is a tree-like structure whose branching points represent probabilistic choices. This is obtained by unfolding from $s$ the graph structure underlying $\mathcal{U}$ and by selecting at each state a single transition of $\mathcal{U}$ - deterministic scheduler - or a convex combination of equally labeled transitions of $\mathcal{U}$ - randomized scheduler - among all the transitions possible from that state. A resolution of $s$ can be formalized as an NPLTS $\mathcal{Z}$ rooted at a state $z_{s}$ corresponding to $s$, in which every state has at most one outgoing transition and hence function $\operatorname{prob}_{\mathrm{m}}$ can be safely applied.

Definition 16. Let $\mathcal{U}=(S, A, \longrightarrow)$ be an NPLTS and $s \in S$. We say that an NPLTS $\mathcal{Z}=(Z, A, \longrightarrow \mathcal{Z})$ is a resolution of $s$ obtained via a deterministic scheduler iff there exists a state correspondence function corr $: Z \rightarrow S$ such that $s=\operatorname{corr}\left(z_{s}\right)$, for some $z_{s} \in Z$, and for all $z \in Z$ :

- If $z \stackrel{a}{\longrightarrow} \mathcal{Z} \mathcal{D}$, then $\operatorname{corr}(z) \stackrel{a}{\longrightarrow} \mathcal{D}^{\prime}$ with $\mathcal{D}\left(z^{\prime}\right)=\mathcal{D}^{\prime}\left(\operatorname{corr}\left(z^{\prime}\right)\right)$ for all $z^{\prime} \in Z$.

- If $z \stackrel{a_{1}}{\longrightarrow} \mathcal{D}_{1}$ and $z \stackrel{a_{2}}{\longrightarrow} \mathcal{D}_{2}$, then $a_{1}=a_{2}$ and $\mathcal{D}_{1}=\mathcal{D}_{2}$.

We denote by Res(s) the set of resolutions of $s$. 
On the basis of the notion above, we provide a $\sim_{\mathrm{PB}, \mathrm{pm}}$-inspired definition of $\sim_{\mathrm{PB} \text {,dis }}$ and show that it coincides with $\sim_{\mathrm{PB} \text {,dis }}$ itself. The ct-variant of the $\sim_{\mathrm{PB}, \mathrm{pm}}$-inspired equivalence can be defined similarly and satisfies an analogous property with respect to the original one-step ct-equivalence.

Definition 17. Let $(S, A, \longrightarrow)$ be an NPLTS. An equivalence relation $\mathcal{B}$ over $S$ is a p-multistep class-distribution probabilistic bisimulation iff, whenever $\left(s_{1}, s_{2}\right)$ $\in \mathcal{B}$, then for all traces $\alpha \in A^{*}$ it holds that for each $z_{s_{1}} \stackrel{\alpha}{\Longrightarrow}$ in a resolution $\mathcal{Z}_{1} \in \operatorname{Res}\left(s_{1}\right)$ there exists $z_{s_{2}} \stackrel{\alpha}{\Longrightarrow}$ in a resolution $\mathcal{Z}_{2} \in \operatorname{Res}\left(s_{2}\right)$ such that for all equivalence classes $C \in S / \mathcal{B}$ :

$$
\operatorname{prob}_{\mathrm{m}}\left(z_{s_{1}}, \alpha, \operatorname{corr}_{\mathcal{Z}_{1}}^{-1}(C)\right)=\operatorname{prob}_{\mathrm{m}}\left(z_{s_{2}}, \alpha, \operatorname{corr}_{\mathcal{Z}_{2}}^{-1}(C)\right)
$$

We denote by $\sim_{\mathrm{PB}, \mathrm{dis}, \mathrm{pm}}$ the largest p-multistep class-distribution probabilistic bisimulation.

Theorem 19. Let $(S, A, \longrightarrow)$ be an NPLTS and $s_{1}, s_{2} \in S$. Then:

$$
s_{1} \sim_{\mathrm{PB}, \mathrm{dis}, \mathrm{pm}} s_{2} \Longleftrightarrow s_{1} \sim_{\mathrm{PB}, \operatorname{dis}} s_{2}
$$

Proof. Suppose that $s_{1} \sim_{\mathrm{PB}, \mathrm{dis}, \mathrm{pm}} s_{2}$. This means that there exists a p-multistep class-distribution probabilistic bisimulation $\mathcal{B}$ over $S$ such that $\left(s_{1}, s_{2}\right) \in \mathcal{B}$. As a consequence, it holds in particular that for all $\left(s_{1}^{\prime}, s_{2}^{\prime}\right) \in \mathcal{B}$ and $a \in A$, whenever $z_{s_{1}} \stackrel{a}{\Longrightarrow}$ in a resolution $\mathcal{Z}_{1} \in \operatorname{Res}\left(s_{1}\right)$, then $z_{s_{2}} \stackrel{a}{\Longrightarrow}$ in a resolution $\mathcal{Z}_{2} \in \operatorname{Res}\left(s_{2}\right)$ such that for all $C \in S / \mathcal{B}$ :

$$
\operatorname{prob}_{\mathrm{m}}\left(z_{s_{1}}, a, \operatorname{corr}_{\mathcal{Z}_{1}}^{-1}(C)\right)=\operatorname{prob}_{\mathrm{m}}\left(z_{s_{2}}, a, \operatorname{corr}_{\mathcal{Z}_{2}}^{-1}(C)\right)
$$

Since $\stackrel{a}{\longrightarrow}$ coincides with $\stackrel{a}{\longrightarrow}$ and for all $s \in S$ such that $z_{s} \stackrel{a}{\longrightarrow} \mathcal{D}$ in a resolution $\mathcal{Z} \in \operatorname{Res}(s)$ it holds that:

$$
\operatorname{prob}_{\mathrm{m}}\left(z_{s}, a, \operatorname{corr}_{\mathcal{Z}}^{-1}(C)\right)=\sum_{z_{s^{\prime}} \in \operatorname{corr}_{\mathcal{Z}}^{-1}(C)} \mathcal{D}\left(z_{s^{\prime}}\right)=\mathcal{D}\left(\operatorname{corr}_{\mathcal{Z}}^{-1}(C)\right)
$$

we have that for each $s_{1}^{\prime} \stackrel{a}{\longrightarrow} \mathcal{D}_{1}$ there exists $s_{2}^{\prime} \stackrel{a}{\longrightarrow} \mathcal{D}_{2}$ such that, for all $C \in$ $S / \mathcal{B}, \mathcal{D}_{1}(C)=\mathcal{D}_{2}(C)$. In other words, $\mathcal{B}$ is also a class-distribution probabilistic bisimulation and hence $s_{1} \sim_{\mathrm{PB}, \mathrm{dis}} s_{2}$.

Suppose now that $s_{1} \sim_{\mathrm{PB} \text {,dis }} s_{2}$. This means that there exists a class-distribution probabilistic bisimulation $\mathcal{B}$ over $S$ such that $\left(s_{1}, s_{2}\right) \in \mathcal{B}$. We prove that $\mathcal{B}$ is also a p-multistep class-distribution probabilistic bisimulation, so that $s_{1} \sim_{\mathrm{PB}, \mathrm{dis}, \mathrm{pm}} s_{2}$ will follow. Given $s_{1}^{\prime}, s_{2}^{\prime} \in S$ such that $\left(s_{1}^{\prime}, s_{2}^{\prime}\right) \in \mathcal{B}$ and $\alpha \in A^{*}$, we proceed by induction on $|\alpha|$ :

- If $|\alpha|=0$, then $z_{s_{1}^{\prime}} \stackrel{\alpha}{\Longrightarrow} \delta_{z_{s_{1}^{\prime}}}$ and $z_{s_{2}^{\prime}} \stackrel{\alpha}{\Longrightarrow} \delta_{z_{s_{2}^{\prime}}}$ are the only possible computations labeled with $\alpha$ in any resolution $\mathcal{Z}_{1} \in \operatorname{Res}\left(s_{1}^{\prime}\right)$ and any resolution $\mathcal{Z}_{2} \in \operatorname{Res}\left(s_{2}^{\prime}\right)$, respectively, and for all $C \in S / \mathcal{B}$ it holds that:

$$
\begin{gathered}
\operatorname{prob}_{\mathrm{m}}\left(z_{s_{1}^{\prime}}, \alpha, \operatorname{corr}_{\mathcal{Z}_{1}}^{-1}(C)\right)=\operatorname{prob}_{\mathrm{m}}\left(z_{s_{2}^{\prime}}, \alpha, \operatorname{corr}_{\mathcal{Z}_{2}}^{-1}(C)\right)= \\
\begin{cases}1 & \text { if }\left\{s_{1}^{\prime}, s_{2}^{\prime}\right\} \subseteq C \\
0 & \text { if }\left\{s_{1}^{\prime}, s_{2}^{\prime}\right\} \cap C=\emptyset\end{cases}
\end{gathered}
$$

because $\left(s_{1}^{\prime}, s_{2}^{\prime}\right) \in \mathcal{B}$ and $C$ is an equivalence class with respect to $\mathcal{B}$.

- Let $|\alpha|=n \in \mathbb{N}_{>0}$ and suppose that the result holds for all traces of length $n-1$. Assume $\alpha=a \alpha^{\prime}$. Since $\left(s_{1}^{\prime}, s_{2}^{\prime}\right) \in \mathcal{B}$ and $\mathcal{B}$ is a class-distribution probabilistic bisimulation, it holds that for each $s_{1}^{\prime} \stackrel{a}{\longrightarrow} \mathcal{D}_{1}$ there exists $s_{2}^{\prime} \stackrel{a}{\longrightarrow} \mathcal{D}_{2}$ 
such that, for all $C \in S / \mathcal{B}, \mathcal{D}_{1}(C)=\mathcal{D}_{2}(C)$.

Given $s \in S$ such that $z_{s} \stackrel{\alpha}{\Longrightarrow}$ with $z_{s} \stackrel{a}{\longrightarrow} \mathcal{D}$ in a resolution $\mathcal{Z} \in \operatorname{Res}(s)$, for all $C \in S / \mathcal{B}$ it holds that:

$$
\begin{aligned}
\operatorname{prob}_{\mathrm{m}}\left(z_{s}, \alpha, \operatorname{corr}_{\mathcal{Z}}^{-1}(C)\right)= & =\sum_{z_{s^{\prime}} \in Z} \mathcal{D}\left(z_{s^{\prime}}\right) \cdot \operatorname{prob}_{\mathrm{m}}\left(z_{s^{\prime}}, \alpha^{\prime}, \operatorname{corr}_{\mathcal{Z}}^{-1}(C)\right) \\
= & \sum_{C^{\prime} \in S / \mathcal{B}} \sum_{z_{s^{\prime}} \in \operatorname{corr}_{\mathcal{Z}}^{-1}\left(C^{\prime}\right)} \mathcal{D}\left(z_{s^{\prime}}\right) \cdot \operatorname{prob}_{\mathrm{m}}\left(z_{s^{\prime}}, \alpha^{\prime}, \operatorname{corr}_{\mathcal{Z}}^{-1}(C)\right) \\
= & \sum_{C^{\prime} \in S / \mathcal{B}} \sum_{z_{s^{\prime}} \in \operatorname{corr}_{\mathcal{Z}}^{-1}\left(C^{\prime}\right)} \mathcal{D}\left(z_{s^{\prime}}\right) \cdot \operatorname{prob}_{\mathrm{m}}\left(z_{s_{C^{\prime}}}, \alpha^{\prime}, \operatorname{corr}_{\mathcal{Z}}^{-1}(C)\right) \\
= & \sum_{C^{\prime} \in S / \mathcal{B}} \operatorname{prob}_{\mathrm{m}}\left(z_{s_{C^{\prime}}}, \alpha^{\prime}, \operatorname{corr}_{\mathcal{Z}}^{-1}(C)\right) \cdot \sum_{z_{s^{\prime}} \in \operatorname{corr}_{\mathcal{Z}}^{-1}\left(C^{\prime}\right)} \mathcal{D}\left(z_{s^{\prime}}\right) \\
= & \sum_{C^{\prime} \in S / \mathcal{B}} \operatorname{prob}_{\mathrm{m}}\left(z_{s_{C^{\prime}}}, \alpha^{\prime}, \operatorname{corr}_{\mathcal{Z}}^{-1}(C)\right) \cdot \mathcal{D}\left(\operatorname{corr}_{\mathcal{Z}}^{-1}\left(C^{\prime}\right)\right)
\end{aligned}
$$

where $s_{C^{\prime}} \in C^{\prime}$ and the factorization of $\operatorname{prob}_{\mathrm{m}}\left(z_{s_{C^{\prime}}}, \alpha^{\prime}, \operatorname{corr}_{\mathcal{Z}}^{-1}(C)\right)$ stems from the application of the induction hypothesis on $\alpha^{\prime}$ to all states of each equivalence class $C^{\prime}$. Since for each $s_{1}^{\prime} \stackrel{a}{\longrightarrow} \mathcal{D}_{1}$ there exists $s_{2}^{\prime} \stackrel{a}{\longrightarrow} \mathcal{D}_{2}$ such that, for all $C^{\prime} \in S / \mathcal{B}, \mathcal{D}_{1}\left(C^{\prime}\right)=\mathcal{D}_{2}\left(C^{\prime}\right)$, we derive that, whenever $z_{s_{1}^{\prime}} \stackrel{\alpha}{\Longrightarrow}$ in a resolution $\mathcal{Z}_{1} \in \operatorname{Res}\left(s_{1}^{\prime}\right)$, then $z_{s_{2}^{\prime}} \stackrel{\alpha}{\Longrightarrow}$ in a resolution $\mathcal{Z}_{2} \in \operatorname{Res}\left(s_{2}^{\prime}\right)$ such that for all $C \in S / \mathcal{B}$ :

$$
\operatorname{prob}_{\mathrm{m}}\left(z_{s_{1}^{\prime}}, \alpha, \operatorname{corr}_{\mathcal{Z}}^{-1}(C)\right)=\operatorname{prob}_{\mathrm{m}}\left(z_{s_{2}^{\prime}}, \alpha, \operatorname{corr}_{\mathcal{Z}}^{-1}(C)\right)
$$

Using the notion of resolution, we can also provide a $\sim_{\mathrm{PB}, \mathrm{pm}}$-inspired definition of each of the six group-by-group probabilistic bisimilarities. The ct-variants of the six $\sim_{\mathrm{PB}, \mathrm{pm}}$-inspired group-by-group probabilistic bisimilarities can be defined similarly.

Definition 18. Let $(S, A, \longrightarrow)$ be an NPLTS and the relational operator $\bowtie \in$ $\{=, \leq, \geq\}$. An equivalence relation $\mathcal{B}$ over $S$ is a p-multistep $\bowtie$-group-by-group probabilistic bisimulation iff, whenever $\left(s_{1}, s_{2}\right) \in \mathcal{B}$, then for all traces $\alpha \in A^{*}$ and groups of equivalence classes $\mathcal{G} \in 2^{S / \mathcal{B}}$ it holds that for each $z_{s_{1}} \stackrel{\alpha}{\Longrightarrow}$ in a resolution $\mathcal{Z}_{1} \in \operatorname{Res}\left(s_{1}\right)$ there exists $z_{s_{2}} \stackrel{\alpha}{\Longrightarrow}$ in a resolution $\mathcal{Z}_{2} \in \operatorname{Res}\left(s_{2}\right)$ such that:

$$
\operatorname{prob}_{\mathrm{m}}\left(z_{s_{1}}, \alpha, \operatorname{corr}_{\mathcal{Z}_{1}}^{-1}(\bigcup \mathcal{G})\right) \bowtie \operatorname{prob}_{\mathrm{m}}\left(z_{s_{2}}, \alpha, \operatorname{corr}_{\mathcal{Z}_{2}}^{-1}(\bigcup \mathcal{G})\right)
$$

We denote by $\sim_{\mathrm{PB}, \mathrm{gbg}, \bowtie, \mathrm{pm}}$ the largest -multistep $\bowtie$-group-by-group probabilistic bisimulation.

Definition 19. Let $(S, A, \longrightarrow)$ be an NPLTS. An equivalence relation $\mathcal{B}$ over $S$ is a p-multistep $\sqcup П$-group-by-group probabilistic bisimulation iff, whenever $\left(s_{1}, s_{2}\right) \in \mathcal{B}$, then for all traces $\alpha \in A^{*}$ and groups of equivalence classes $\mathcal{G} \in 2^{S / \mathcal{B}}$ it holds that $s_{1} \stackrel{\alpha}{\Longrightarrow}$ implies $s_{2} \stackrel{\alpha}{\Longrightarrow}$ with: 


$$
\begin{gathered}
\bigsqcup \operatorname{prob}_{\mathrm{m}}\left(z_{s_{1}}, \alpha, \operatorname{corr}_{\mathcal{Z}_{1}}^{-1}(\bigcup \mathcal{G})\right)= \\
\mathcal{Z}_{1} \in \operatorname{Res}\left(s_{1}\right) \text { s.t. } z_{s_{1}} \stackrel{\alpha}{\Longrightarrow} \\
\mathcal{Z}_{2} \in \operatorname{Res}\left(s_{2}\right) \text { s.t. } z_{s_{2}} \stackrel{\alpha}{\Longrightarrow} \\
\prod \operatorname{prob}_{\mathrm{m}}\left(z_{s_{2}}, \alpha, \operatorname{corr}_{\mathcal{Z}_{2}}^{-1}(\bigcup \mathcal{G})\right) \\
\left.\mathcal{Z}_{1} \in \operatorname{Res}\left(s_{1}\right) \text { s.t. } z_{s_{1}} \stackrel{\alpha}{\Longrightarrow}, \alpha, \operatorname{corr}_{\mathcal{Z}_{1}}^{-1}(\bigcup \mathcal{G})\right)= \\
\mathcal{Z}_{2} \in \operatorname{Res}\left(s_{2}\right) \text { s.t. } z_{s_{2}} \stackrel{\alpha}{\Longrightarrow}
\end{gathered}
$$

We denote by $\sim_{\mathrm{PB}, \mathrm{gbg}, \sqcup \sqcap, \mathrm{pm}}$ the largest p-multistep $\sqcup П$-group-by-group probabilistic bisimulation.

Definition 20. Let $(S, A, \longrightarrow)$ be an NPLTS and symbol \# $\in\{\bigsqcup, \Pi\}$. An equivalence relation $\mathcal{B}$ over $S$ is a p-multistep \#-group-by-group probabilistic bisimulation iff, whenever $\left(s_{1}, s_{2}\right) \in \mathcal{B}$, then for all traces $\alpha \in A^{*}$ and groups of equivalence classes $\mathcal{G} \in 2^{S / \mathcal{B}}$ it holds that $s_{1} \stackrel{\alpha}{\Longrightarrow}$ implies $s_{2} \stackrel{\alpha}{\Longrightarrow}$ with:

$$
\begin{aligned}
& \# \operatorname{prob}_{\mathrm{m}}\left(z_{s_{1}}, \alpha, \operatorname{corr}_{\mathcal{Z}_{1}}^{-1}(\bigcup \mathcal{G})\right)= \\
& \mathcal{Z}_{1} \in \operatorname{Res}\left(s_{1}\right) \text { s.t. } z_{s_{1}} \stackrel{\alpha}{\Longrightarrow} \\
& \mathcal{Z}_{2} \in \operatorname{Res}\left(s_{2}\right) \text { s.t. } z_{s_{2}} \stackrel{\alpha}{\Longrightarrow} \\
& \text { \# } \operatorname{prob}_{\mathrm{m}_{\alpha}}\left(z_{s_{2}}, \alpha, \operatorname{corr}_{\mathcal{Z}_{2}}^{-1}(\cup \mathcal{G})\right)
\end{aligned}
$$

We denote by $\sim_{\mathrm{PB}, \mathrm{gbg}, \#, \mathrm{pm}}$ the largest -multistep \#-group-by-group probabilistic bisimulation.

The six $\sim_{\mathrm{PB}, \mathrm{pm}}$-inspired group-by-group probabilistic bisimilarities can be alternatively defined without making explicit use of the notion of resolution. Given $s \in S, \alpha \in A^{*}$, and $S^{\prime} \subseteq S$, we inductively define the set of multistep probabilities of reaching a state in $S^{\prime}$ from $s$ via $\alpha$ as follows:

$$
\text { probset }_{\mathrm{m}}\left(s, \alpha, S^{\prime}\right)=\left\{\begin{array}{lr}
\bigcup_{a}\left\{\sum_{s^{\prime} \in S} \mathcal{D}\left(s^{\prime}\right) \cdot p_{s^{\prime}} \mid p_{s^{\prime}} \in \text { probset }_{\mathrm{m}}\left(s^{\prime}, \alpha^{\prime}, S^{\prime}\right)\right\} \\
\{1\} & \text { if } \alpha=a \alpha^{\prime} \text { and } s \stackrel{a}{\longrightarrow} \\
\{0\} & \text { if } \alpha=\varepsilon \text { and } s \in S^{\prime} \\
& \text { if } \alpha=a \alpha^{\prime} \text { and } s \stackrel{a}{\rightarrow} \\
& \text { or } \alpha=\varepsilon \text { and } s \notin S^{\prime}
\end{array}\right.
$$

Since $\operatorname{probset}_{\mathrm{m}}\left(s, \alpha, S^{\prime}\right)=\left\{\operatorname{prob}_{\mathrm{m}}\left(z_{s}, \alpha, \operatorname{corr}_{\mathcal{Z}}^{-1}\left(S^{\prime}\right)\right) \mid \mathcal{Z} \in \operatorname{Res}(s)\right\}$, it is easy to see that in Defs. 18 to 20 we could have used $\operatorname{probset}_{\mathrm{m}}\left(s_{i}, \alpha, \bigcup \mathcal{G}\right)$ in place of $\operatorname{prob}_{\mathrm{m}}\left(z_{s_{i}}, \alpha, \operatorname{corr}_{\mathcal{Z}_{i}}^{-1}(\bigcup \mathcal{G})\right)$ for $i=1,2$. This is not possible in Def. 17 because the use of probset $_{\mathrm{m}}$ causes the connection between each computation and the resolution to which it belongs to be broken.

Each of the six $\sim_{\mathrm{PB}, \mathrm{pm}}$-inspired group-by-group probabilistic bisimilarities is contained in the corresponding original one-step equivalence. The ct-variants of the six $\sim_{\mathrm{PB}, \mathrm{pm}}$-inspired group-by-group probabilistic bisimilarities satisfy an analogous inclusion property with respect to the original one-step ct-equivalences.

Theorem 20. Let $(S, A, \longrightarrow)$ be an NPLTS, $s_{1}, s_{2} \in S$, and $\circ \in\{=, \leq, \geq$, $\sqcup \sqcap, \sqcup, \sqcap\}$. Then:

$$
s_{1} \sim_{\mathrm{PB}, \mathrm{gbg}, \circ, \mathrm{pm}} s_{2} \Longrightarrow s_{1} \sim_{\mathrm{PB}, \mathrm{gbg}, \circ} s_{2}
$$


Proof. Let $\circ=\bowtie \in\{=, \leq, \geq\}$ and suppose that $s_{1} \sim_{\mathrm{PB}, \mathrm{gbg}, \bowtie, \mathrm{pm}} s_{2}$. This means that there exists a p-multistep $\bowtie$-group-by-group probabilistic bisimulation $\mathcal{B}$ over $S$ such that $\left(s_{1}, s_{2}\right) \in \mathcal{B}$. As a consequence, it holds in particular for all $\left(s_{1}^{\prime}, s_{2}^{\prime}\right) \in \mathcal{B}, a \in A$, and $\mathcal{G} \in 2^{S / \mathcal{B}}$ that for each $z_{s_{1}^{\prime}} \stackrel{a}{\Longrightarrow}$ in a resolution $\mathcal{Z}_{1} \in \operatorname{Res}\left(s_{1}^{\prime}\right)$ there exists $z_{s_{2}^{\prime}} \stackrel{a}{\Longrightarrow}$ in a resolution $\mathcal{Z}_{2} \in \operatorname{Res}\left(s_{2}^{\prime}\right)$ such that:

$$
\operatorname{prob}_{\mathrm{m}}\left(z_{s_{1}^{\prime}}, a, \operatorname{corr}_{\mathcal{Z}_{1}}^{-1}(\bigcup \mathcal{G})\right) \bowtie \operatorname{prob}_{\mathrm{m}}\left(z_{s_{2}^{\prime}}, a, \operatorname{corr}_{\mathcal{Z}_{2}}^{-1}(\bigcup \mathcal{G})\right)
$$

Since $\stackrel{a}{\longrightarrow}$ coincides with $\stackrel{a}{\longrightarrow}$ and for all $s \in S$ such that $z_{s} \stackrel{a}{\longrightarrow} \mathcal{D}$ in a resolution $\mathcal{Z} \in \operatorname{Res}(s)$ it holds that:

$$
\operatorname{prob}_{\mathrm{m}}\left(z_{s}, a, \operatorname{corr}_{\mathcal{Z}}^{-1}(\bigcup \mathcal{G})\right)=\sum_{z_{s^{\prime}} \in \operatorname{corr}_{\mathcal{Z}}^{-1}(\bigcup \mathcal{G})} \mathcal{D}\left(z_{s^{\prime}}\right)=\mathcal{D}\left(\operatorname{corr}_{\mathcal{Z}}^{-1}(\bigcup \mathcal{G})\right)
$$

we have that for each $s_{1}^{\prime} \stackrel{a}{\longrightarrow} \mathcal{D}_{1}$ there exists $s_{2}^{\prime} \stackrel{a}{\longrightarrow} \mathcal{D}_{2}$ with $\mathcal{D}_{1}(\cup \mathcal{G}) \bowtie \mathcal{D}_{2}(\bigcup \mathcal{G})$. In other words, $\mathcal{B}$ is also a $\bowtie$-group-by-group probabilistic bisimulation and hence $s_{1} \sim \mathrm{PB}, \mathrm{gbg}, \bowtie s_{2}$.

Suppose now that $s_{1} \sim_{\mathrm{PB}, \mathrm{gbg}, \sqcup \sqcap, \mathrm{pm}} s_{2}$. This means that there exists a p-multistep $\sqcup \sqcap$-group-by-group probabilistic bisimulation $\mathcal{B}$ over $S$ such that $\left(s_{1}, s_{2}\right) \in \mathcal{B}$. As a consequence, it holds in particular for all $\left(s_{1}^{\prime}, s_{2}^{\prime}\right) \in \mathcal{B}, a \in A$, and $\mathcal{G} \in 2^{S / \mathcal{B}}$ that, whenever $s_{1}^{\prime} \stackrel{a}{\Longrightarrow}$, then $s_{2}^{\prime} \stackrel{a}{\Longrightarrow}$ with:

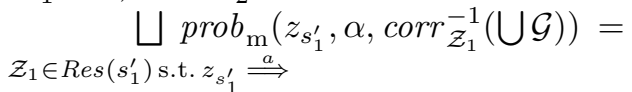

$$
\begin{aligned}
& \underset{\mathcal{Z}_{2} \in \operatorname{Res}\left(s_{2}^{\prime}\right) \text { s.t. } z_{s_{2}^{\prime}} \stackrel{a}{\Longrightarrow}}{\operatorname{mrob}_{\mathrm{m}}\left(z_{s_{2}^{\prime}}, \alpha, \operatorname{corr}_{\mathcal{Z}_{2}}^{-1}(\bigcup \mathcal{G})\right)} \\
& \prod \operatorname{prob}_{\mathrm{m}}\left(z_{s_{1}^{\prime}}, \alpha, \operatorname{corr}_{\mathcal{Z}_{1}}^{-1}(\bigcup \mathcal{G})\right)= \\
& \mathcal{Z}_{1} \in \operatorname{Res}\left(s_{1}^{\prime}\right) \text { s.t. } z_{s_{1}^{\prime}} \stackrel{a}{\Longrightarrow} \\
& \prod_{\mathcal{Z}_{2} \in \operatorname{Res}\left(s_{2}^{\prime}\right) \text { s.t. } z_{s_{2}^{\prime}} \stackrel{a}{\Longrightarrow}}
\end{aligned}
$$

Since $\stackrel{a}{\Longrightarrow}$ coincides with $\stackrel{a}{\longrightarrow}$ and for all $s \in S$ such that $z_{s} \stackrel{a}{\longrightarrow} \mathcal{D}$ in a resolution $\mathcal{Z} \in \operatorname{Res}(s)$ it holds that:

$$
\operatorname{prob}_{\mathrm{m}}\left(z_{s}, a, \operatorname{corr}_{\mathcal{Z}}^{-1}(\bigcup \mathcal{G})\right)=\sum_{z_{s^{\prime}} \in \operatorname{corr}_{\mathcal{Z}}^{-1}(\bigcup \mathcal{G})} \mathcal{D}\left(z_{s^{\prime}}\right)=\mathcal{D}\left(\operatorname{corr}_{\mathcal{Z}}^{-1}(\bigcup \mathcal{G})\right)
$$

we have that $s_{1}^{\prime} \stackrel{a}{\longrightarrow}$ implies $s_{2}^{\prime} \stackrel{a}{\longrightarrow}$ with:

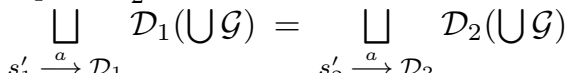

$$
\begin{aligned}
& \prod_{s_{1}^{\prime} \stackrel{s_{1}^{\prime}}{\longrightarrow} \mathcal{D}_{1}} \mathcal{D}_{1}(\bigcup \mathcal{G})=\prod_{s_{2}^{\prime} \stackrel{\prod_{a}}{\longrightarrow} \mathcal{D}_{2}}^{s_{2}^{\prime}} \mathcal{D}_{2}(\bigcup \mathcal{G})
\end{aligned}
$$

In other words, $\mathcal{B}$ is also a பП-group-by-group probabilistic bisimulation and hence $s_{1} \sim \mathrm{PB}$,gbg,பП $s_{2}$.

Finally, the proof that $s_{1} \sim_{\mathrm{PB}, \mathrm{gbg}, \#, \mathrm{pm}} s_{2}$ implies $s_{1} \sim_{\mathrm{PB}, \mathrm{gbg}, \#} s_{2}$ for $\# \in\{\sqcup, \sqcap\}$ is similar to the proof that $s_{1} \sim_{\mathrm{PB}, \mathrm{gbg}, \sqcup \sqcap, \mathrm{pm}} s_{2}$ implies $s_{1} \sim_{\mathrm{PB}, \mathrm{gbg}, \sqcup \sqcap} s_{2}$.

Unlike Thm. 19, the reverse implication of Thm. 20 does not hold in general. For example, in Fig. 6 we have that $s_{1} \sim_{\mathrm{PB}, \mathrm{gbg},=} s_{2}$ but $s_{1} \chi_{\mathrm{PB}, \mathrm{gbg},=, \mathrm{pm}} s_{2}$ because, for $\alpha=a b c$ and $\mathcal{G}$ containing all the states with no outgoing transitions, it turns out that the multistep probability of reaching $\mathcal{G}$ via $\alpha$ in the maximal resolution of $s_{1}$ starting with the rightmost $a$-transition - which is $0.1 \cdot 0.7+0.9$. 

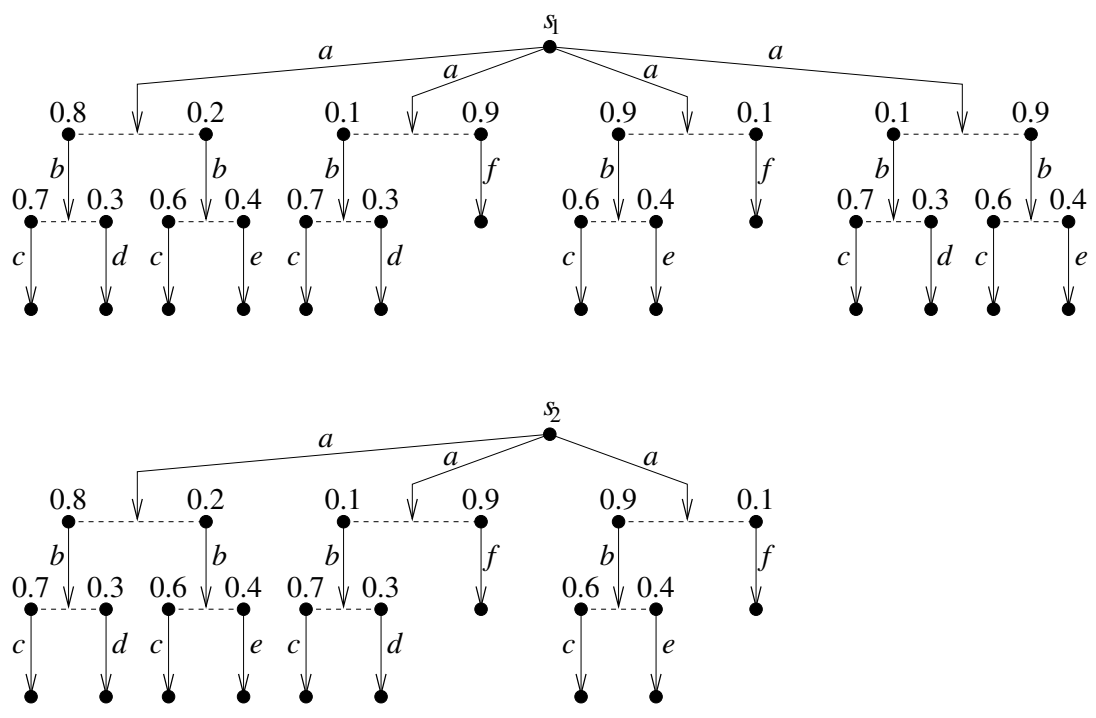

Fig. 6. Two models related by $\sim_{\mathrm{PB}, \mathrm{gbg},=}$ that are distinguished by $\sim_{\mathrm{PB}, \mathrm{gbg},=, \mathrm{pm}}$

$0.6=0.61-$ is not matched by any of the multistep probabilities of reaching $\mathcal{G}$ via $\alpha$ in the three maximal resolutions of $s_{2}$ starting with the three $a$-transitions - which are $0.8 \cdot 0.7+0.2 \cdot 0.6=0.68,0.1 \cdot 0.7=0.07$, and $0.9 \cdot 0.6=0.54$.

We conclude by showing that all the considered $\sim_{\mathrm{PB}, \mathrm{pm}}$-inspired probabilistic bisimilarities collapse into $\sim_{\mathrm{PB}, \mathrm{pm}}$ when restricting attention to reactive probabilistic processes. An analogous result holds for their ct-variants.

Theorem 21. Let $(S, A, \longrightarrow)$ be an NPLTS in which the transitions of each state have different labels. Let $s_{1}, s_{2} \in S$ and $\circ \in\{=, \leq, \geq, \sqcup \sqcap, \sqcup, \sqcap\}$. Then:

$$
s_{1} \sim_{\mathrm{PB}, \mathrm{dis}, \mathrm{pm}} s_{2} \Longleftrightarrow s_{1} \sim_{\mathrm{PB}, \mathrm{gbg}, \mathrm{\circ}, \mathrm{pm}} s_{2} \Longleftrightarrow s_{1} \sim_{\mathrm{PB}, \mathrm{pm}} s_{2}
$$

Proof. Since every state of this specific NPLTS has at most one transition labeled with a certain action, a p-multistep probabilistic bisimulation is trivially a p-multistep $=$-group-by-group probabilistic bisimulation, a $p$-multistep $\leq$-groupby-group probabilistic bisimulation, a p-multistep $\geq$-group-by-group probabilistic bisimulation, a p-multistep $\sqcup П$-group-by-group probabilistic bisimulation, a $p$ multistep $\sqcup$-group-by-group probabilistic bisimulation, and a p-multistep $\sqcap$-groupby-group probabilistic bisimulation. 